INL REPORT

INL/EXT-14-33977

Unlimited Release

Printed December 2014

\title{
RELAP-7 User's Guide
}

Prepared by

Idaho National Laboratory

Idaho Falls, Idaho 83415

The Idaho National Laboratory is a multiprogram laboratory operated by Battelle Energy Alliance for the United States Department of Energy under DOE Idaho Operations Office. Contract DE-AC07-05ID14517.

Approved for public release; further dissemination unlimited.

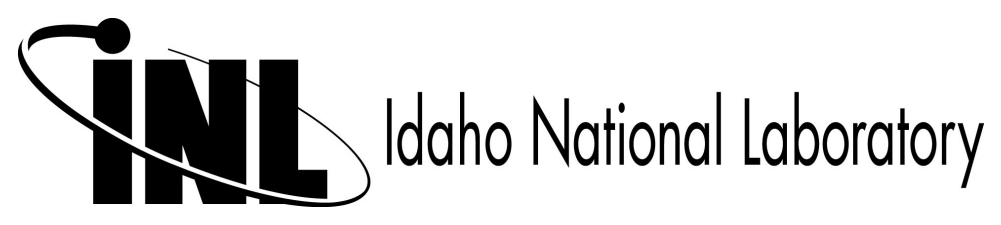


Issued by the Idaho National Laboratory, operated for the United States Department of Energy by Battelle Energy Alliance.

NOTICE: This report was prepared as an account of work sponsored by an agency of the United States Government. Neither the United States Government, nor any agency thereof, nor any of their employees, nor any of their contractors, subcontractors, or their employees, make any warranty, express or implied, or assume any legal liability or responsibility for the accuracy, completeness, or usefulness of any information, apparatus, product, or process disclosed, or represent that its use would not infringe privately owned rights. Reference herein to any specific commercial product, process, or service by trade name, trademark, manufacturer, or otherwise, does not necessarily constitute or imply its endorsement, recommendation, or favoring by the United States Government, any agency thereof, or any of their contractors or subcontractors. The views and opinions expressed herein do not necessarily state or reflect those of the United States Government, any agency thereof, or any of their contractors.

Printed in the United States of America. This report has been reproduced directly from the best available copy.

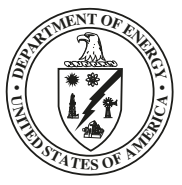


INL/EXT-14-33977

Unlimited Release

Printed December 2014

\section{RELAP-7 User's Guide}

Hongbin Zhang, Haihua Zhao, Ling Zou, David Andrs, Ray Berry, Richard Martineau 


\section{Contents}

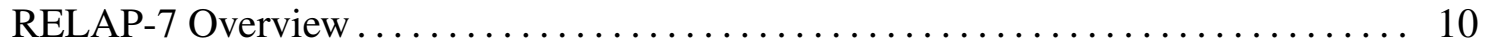

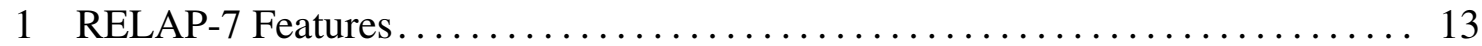

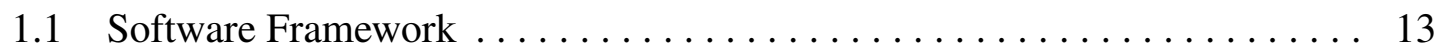

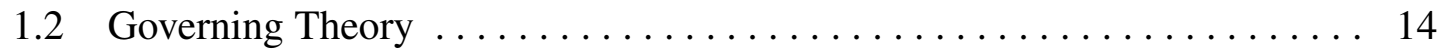

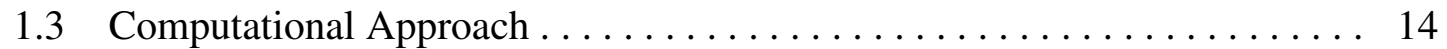

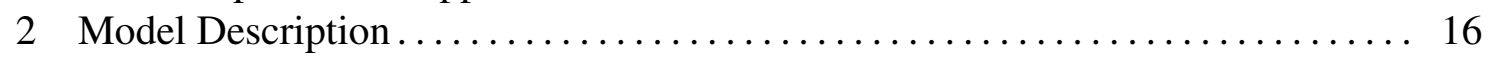

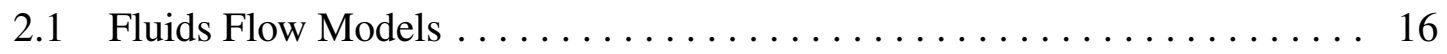

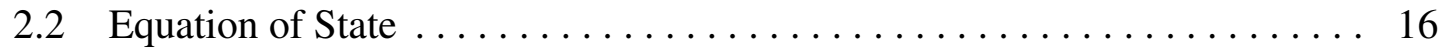

2.3 Solution Stabilization Schemes $\ldots \ldots \ldots \ldots \ldots \ldots \ldots \ldots \ldots \ldots \ldots$

2.4 Time Integration Schemes $\ldots \ldots \ldots \ldots \ldots \ldots \ldots \ldots \ldots \ldots \ldots \ldots \ldots$

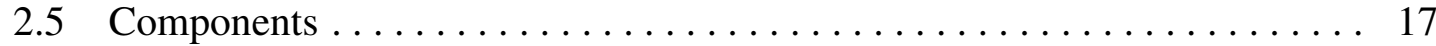

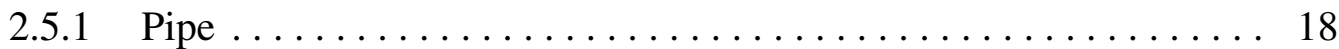

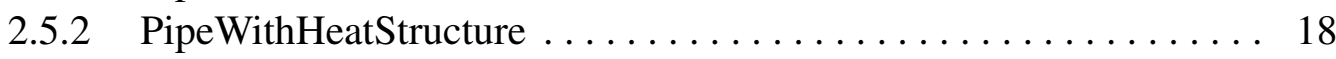

2.5.3 CoreChannel . . . . . . . . . . . . . . . . . . . . . . 18

2.5.4 Subchannel . . . . . . . . . . . . . . . . . . . . . . . . . . . . . . 19

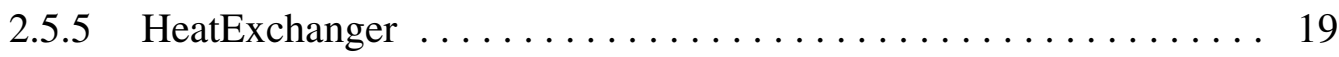

2.5 .6 Branch ................................ 19

2.5.7 VolumeBranch ... . . . . . . . . . . . . . . . . . . . . . . . . . . 19

2.5.8 SubchannelBranch.......................... 19

2.5 .9 Pump . . . . . . . . . . . . . . . . . . . . . . . . 20

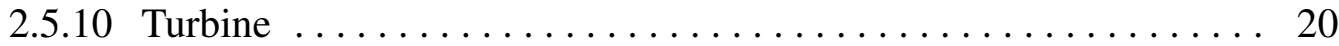

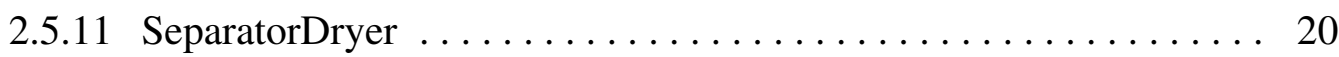

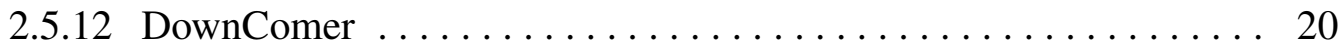

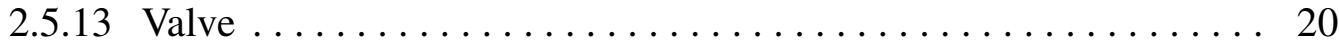

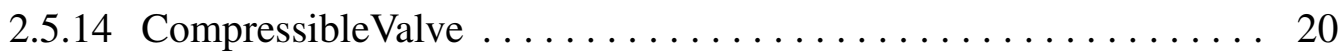

2.5.15 Check Valves ........................ 21

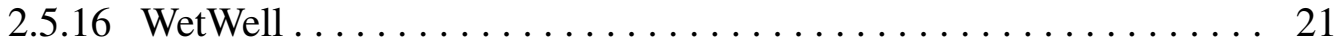

2.5.17 TimeDependentVolume ..................... 21

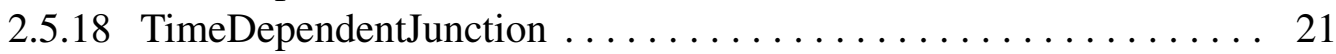

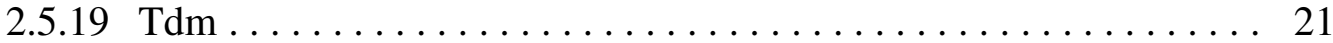

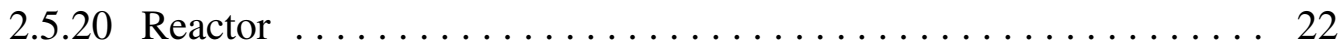

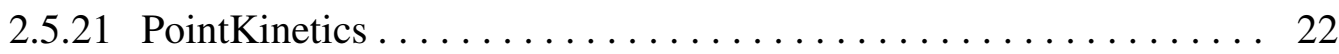

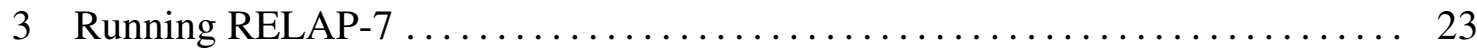

3.1 Complete Step 1 of MOOSE Environment Setup . . . . . . . . . . . 23

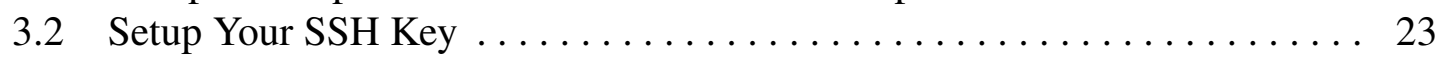

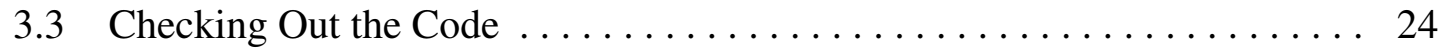




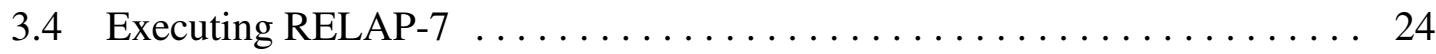

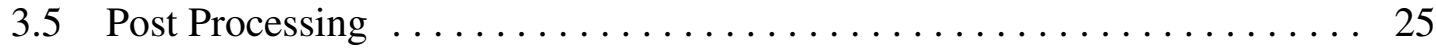

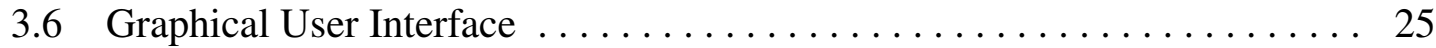

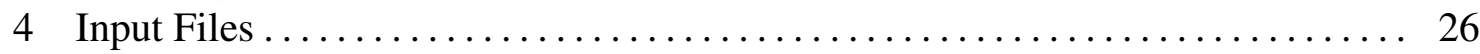

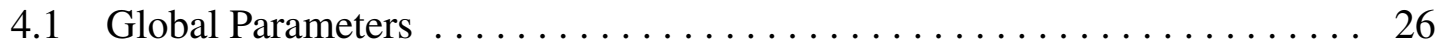

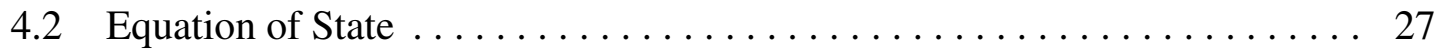

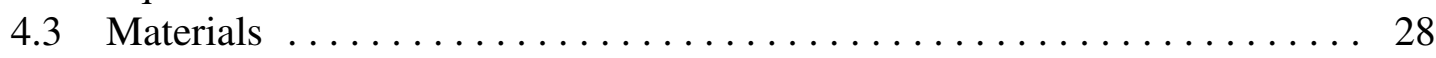

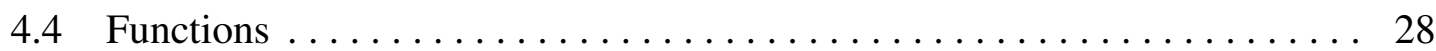

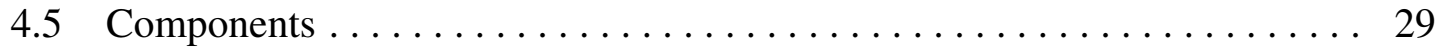

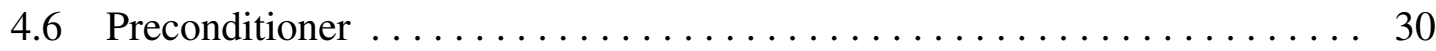

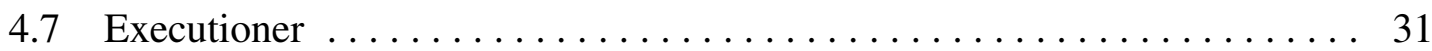

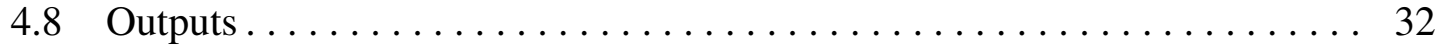

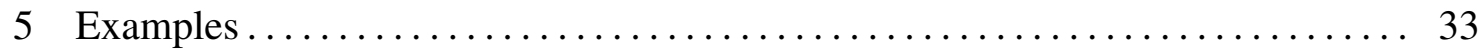

5.1 Example 1: A Simple Pipe Flow Problem ............... 33

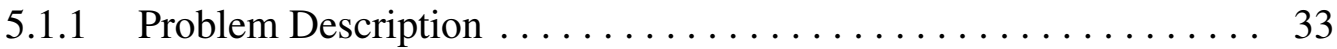

$5.1 .2 \quad$ Input File . . . . . . . . . . . . . . . . . . . 34

5.1 .3 Description of the Input File . . . . . . . . . . . . 37

5.2 Example 2: Use Functions to Set Initial Conditions for a Simple Pipe Flow

Problem . . . . . . . . . . . . . . . . . . . . . . . . . 44

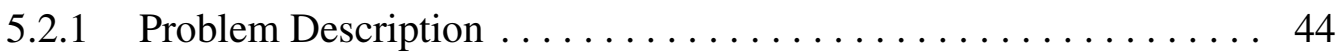

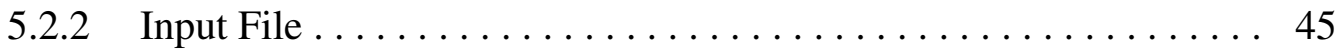

5.2 .3 Description of the Input File $\ldots \ldots \ldots \ldots \ldots \ldots \ldots \ldots \ldots$

5.3 Example 3: Two Phase Flow Through a Pipe - HEM Model . . . . . . . . 52

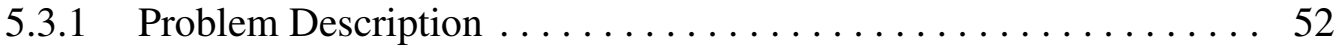

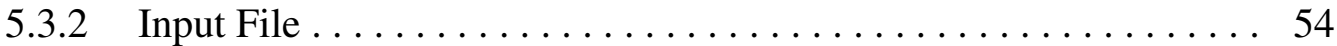

5.3 .3 Description of the Input File $\ldots \ldots \ldots \ldots \ldots \ldots \ldots \ldots$

5.4 Example 4: Two Phase Flow Through a Pipe - 7-Equation Model . . . . . . 60

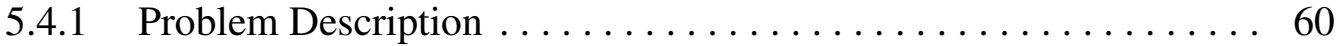

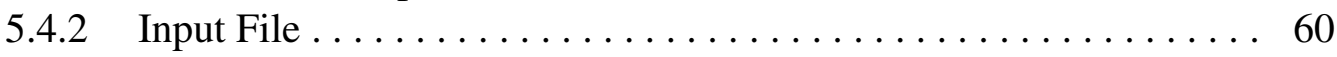

5.4 .3 Description of the Input File . . . . . . . . . . . . . . . 64

5.5 Example 5: A Core Channel Problem . . . . . . . . . . . . . . . . . 69

5.5.1 Problem Description . . . . . . . . . . . . . . . . . 69

5.5 .2 Input File . . . . . . . . . . 71

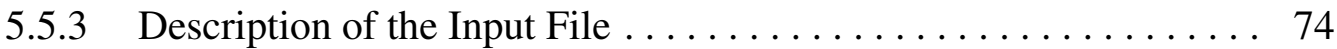

5.6 Example 6: A Two Pipes Flow Problem . . . . . . . . . . . . . 78

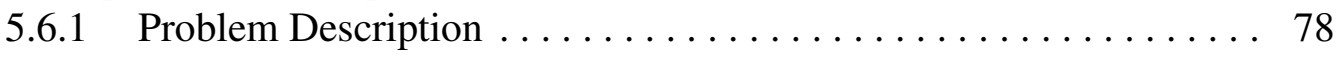

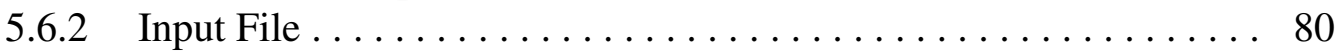

5.6 .3 Description of the Input File $\ldots \ldots \ldots \ldots \ldots \ldots \ldots \ldots \ldots$ 
5.7 Example 7: A Volume Branch Case - Three Pipes In and Two Pipes Out. . 84

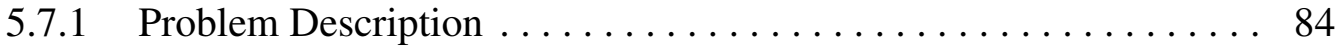

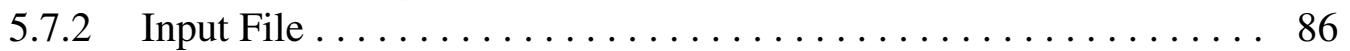

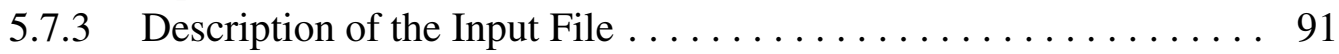

5.8 Example 8: A Simple Pipe Loop with Pump . . . . . . . . . . . . . . 92

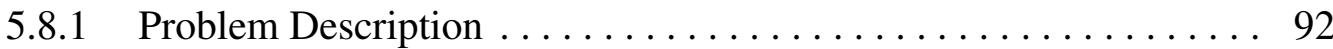

5.8 .2 Input File . . . . . . . . . . . . . . . . . . . 93

5.8 .3 Description of the Input File $\ldots \ldots \ldots \ldots \ldots \ldots \ldots \ldots \ldots$

5.9 Example 9: A Heat Exchanger Problem . . . . . . . . . . . . . . . . . . 99

5.9 .1 Problem Description . . . . . . . . . . . . . . . . . . . 99

5.9 .2 Input File . . . . . . . . . . . . . . . . . . . 100

5.9 .3 Description of the Input File . . . . . . . . . . . . . . . . . . . 104

5.10 Example 10: A Loop With Core Channel and Heat Exchanger . . . . . . . . . 107

5.10 .1 Problem Description . . . . . . . . . . . . . . . . . . . 107

5.10 .2 Input File . . . . . . . . . . . . . . . . . . . . . 110

5.11 Example 11: A Model Pressurized Water Reactor Problem . . . . . . . . . . 118

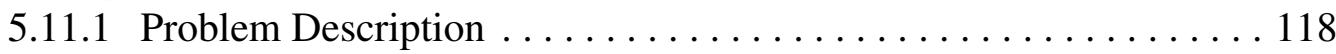

5.11 .2 Input File . . . . . . . . . . . . . . . . . . . . . 119

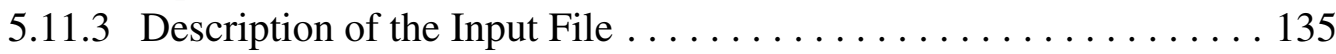

5.12 Example 12: A Simplified Primary System Model of a Boiling Water Reactor 137

5.12 .1 Problem Description . . . . . . . . . . . . . . . . . . 137

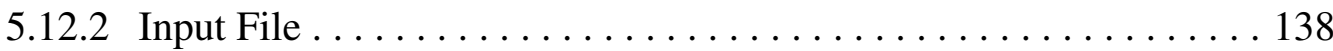

5.12 .3 Description of the Input File $\ldots \ldots \ldots \ldots \ldots \ldots \ldots \ldots \ldots \ldots$

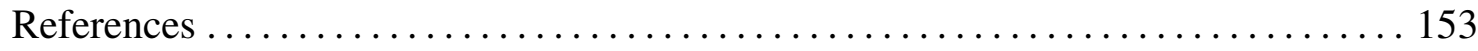




\section{Figures}

1 A simple pipe flow problem diagram .................... 33

2 Pressure vs. length for the simple pipe flow problem $\ldots \ldots \ldots \ldots \ldots .34$

3 Pressure vs length for an isothermal pipe flow problem with initial pressure

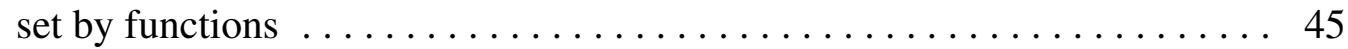

4 Diagram of a core channel problem ....................... 69

5 Diagram of a two pipes flow problem $\ldots \ldots \ldots \ldots \ldots \ldots \ldots \ldots \ldots \ldots$

6 Pressure vs. length for the two pipes flow problem ............... 79

7 Diagram of a volume branch case with three pipes flowing in and two pipes

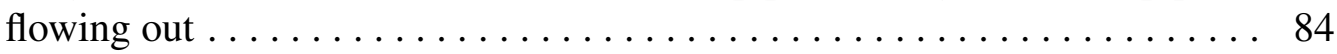

8 Diagram of a simple loop of pipes connected by branches and a pump . . . 92

9 Diagram of a heat exchanger problem ...................... 99

10 Diagram of a loop with core channel and heat exchanger . . . . . . . . . 107

11 Diagram of a model pressurized water reactor problem $\ldots \ldots \ldots \ldots \ldots 118$

12 Diagram of a simplified primary system of a BWR model ........... 137 
Tables 


\section{RELAP-7 Overview}

The RELAP-7 code is the next generation nuclear reactor system safety analysis code being developed at the Idaho National Laboratory (INL). The code is based on the INL's modern scientific software development framework, MOOSE (Multi-Physics Object Oriented Simulation Environment). The overall design goal of RELAP-7 is to take advantage of the previous thirty years of advancements in computer architecture, software design, numerical integration methods, and physical models. The end result will be a reactor systems analysis capability that retains and improves upon RELAP5's capability and extends the analysis capability for all reactor system simulation scenarios.

RELAP-7 will become the main reactor systems simulation toolkit for the LWRS (Light Water Reactor Sustainability) program's RISMC (Risk Informed Safety Margin Characterization) effort and the next generation tool in the RELAP reactor safety/systems analysis application series. The key to the success of RELAP-7 is the simultaneous advancement of physical models, numerical methods, and software design while maintaining a solid user perspective. Physical models include both PDEs (Partial Differential Equations) and ODEs (Ordinary Differential Equations) and experimental based closure models. RELAP-7 utilizes well-posed governing equations for compressible two-phase flow, which can be strictly verified in a modern verification and validation effort. Closure models used in RELAP5 and newly developed models will be reviewed and selected to reflect the progress made during the past three decades and provide a basis for the closure relations that will be required in RELAP-7. RELAP-7 uses modern numerical methods, which allow implicit time integration, second-order schemes in both time and space, and strongly coupled multi-physics.

RELAP-7 is written with object oriented programming language $\mathrm{C}++$. By using the MOOSE development environment, the RELAP-7 code is developed by following the same modern software design paradigms used for other MOOSE development efforts. The code is easy to read, develop, maintain, and couple with other codes. Most importantly, the modern software design allows the RELAP-7 code to evolve efficiently with time. MOOSE is an HPC development and runtime framework for solving computational engineering problems in a well planned, managed, and coordinated way. By leveraging millions of lines of open source software packages, such as PETSc (a nonlinear solver developed at Argonne National Laboratory) and LibMesh (a Finite Element Analysis package developed at University of Texas), MOOSE reduces the expense and time required to develop new applications. MOOSE provides numerical integration methods and mesh management for parallel computation. Therefore RELAP-7 code developers have been 
able to focus more upon the physics and user interface capability. There are currently over 20 different MOOSE based applications ranging from 3-D transient neutron transport, detailed 3-D transient fuel performance analysis, to long-term material aging. Multiphysics and multi-dimensional analysis capabilities, such as radiation transport and fuel performance, can be obtained by coupling RELAP-7 and other MOOSE-based applications through MOOSE and by leveraging with capabilities developed by other DOE programs. This allows restricting the focus of RELAP-7 to systems analysis type simulations and gives priority to retain and significantly extend RELAP5's capabilities.

This document provides a user's guide to help users to learn how to run the RELAP-7 code. A number of example problems and their associated input files are presented in this document to guide users to run the RELAP-7 code starting with simple pipe problems to problems with increasing complexity. Because the code is an ongoing development effort, this RELAP-7 User's Guide will evolve with periodic updates to keep it current with the state of the development, implementation, and model additions/revisions. A complete User's Manual will be developed at a later time when the RELAP-7 code becomes more mature. 


\section{RELAP-7 Features}

An overall description of the RELAP-7 architecture, governing theory, and computational approach is given here as an instructive, and executive overview of the RELAP-7 distinguishing features.

\subsection{Software Framework}

MOOSE is INL's development and runtime environment for the solution of multi-physics systems that involve multiple physical models or multiple simultaneous physical phenomena. The systems are generally represented (modeled) as a system of fully coupled nonlinear partial differential equation systems (an example of a multi-physics system is the thermal feedback effect upon neutronics cross-sections where the cross-sections are a function of the heat transfer). Inside MOOSE, the Jacobian-Free Newton Krylov (JFNK) method [1,2] is implemented as a parallel nonlinear solver that naturally supports effective coupling between physics equation systems (or Kernels). The physics Kernels are designed to contribute to the nonlinear residual, which is then minimized inside of MOOSE. MOOSE provides a comprehensive set of finite element support capabilities (LibMesh [3], a Finite Element library developed at University of Texas) and provides for mesh adaptation and parallel execution. The framework heavily leverages software libraries from DOE SC and NNSA, such as the nonlinear solver capabilities in either the the Portable, Extensible Toolkit for Scientific Computation (PETSc [4]) project or the Trilinos project [5] (a collection of numerical methods libraries developed at Sandia National Laboratory). Argonne's PETSc group has recently joined with the MOOSE team in a strong collaboration wherein they are customizing PETSc for our needs. This collaboration is strong enough that Argonne is viewed as a joint developer of MOOSE.

A parallel and tightly coordinated development effort with the RELAP-7 development project is the Reactor Analysis Virtual control ENvironment (RAVEN). This MOOSEbased application is a complex, multi-role software tool that will have several diverse tasks including serving as the RELAP-7 graphical user interface, using RELAP-7 to perform RISMC focused analysis, and controlling the RELAP-7 calculation execution.

Together, MOOSE/RELAP-7/RAVEN comprise the systems analysis capability of LWRS RISMC ToolKit. 


\subsection{Governing Theory}

The primary basis of the RELAP-7 governing theory includes thermal fluids flow, reactor core heat transfer, and reactor kinetics models.

With respect to the thermal fluids flow dynamics models, RELAP-7 incorporates both single- and two-phase flow simulation capabilities encompassing all-speed and all-fluids. The single phase flow models include isothermal flow and nonisotherml flow capabilities. The two-phase flow models include the homogeneous equilibrium flow model (HEM) and the well posed two fluid 7-equation model.

In addition to the fluids flow dynamics model, RELAP-7 necessarily simulates the heat transfer process with reactor kinetics as the heat source. The heat-conduction equation for cylindrical or slab geometries is solved to provide thermal history within metal structures such as fuel and clad. The volumetric power source in the heat conduction equation for the fuel comes from the point kinetics model with thermal hydraulic reactivity feedback considered [6]. The reactor structure is coupled with the thermal fluid through energy exchange (conjugate heat transfer) employing surface convective heat transfer [7] within the fluid. The fluid, heat conduction, conjugate heat transfer and point kinetics equations are solved in a fully coupled fashion in RELAP-7 in contrast to the operator-splitting or loose coupling approach used in the existing system safety analysis codes.

\subsection{Computational Approach}

Stated previously, the MOOSE framework provides the bulk of the "heavy lifting" available to MOOSE-based applications with a multitude of mathematical and numerical libraries. For RELAP-7, LibMesh [3] provides the second-order accurate spatial discretization by employing linear basis, one-dimensional finite elements. The Message Passing Interface (MPI, from Argonne National Laboratory) provides for distributed parallel processing. Intel Threading Building Blocks (Intel TBB) allows parallel $\mathrm{C}++$ programs to take full advantage of multicore architecture found in most large-scale machines of today. PETSc (from Argonne), Trilinos (from Sandia), and Hypre [8] (from Lawrence Livermore National Laboratory) provide the mathematical libraries and nonlinear solver capabilities for the Jacobian-free Newton-Krylov (JFNK) method. In MOOSE, a stiffly-stable, secondorder backward difference (BDF2) formulation is used to provide second-order accurate time integration for strongly coupled physics in JFNK. 
The JFNK method easily allows implicit nonlinear coupling of dependent physics under one general computational framework. Besides rapid (second-order) convergence of the iterative procedure, the JFNK method flexibly handles multiphysics problems when time scales of different physics are significantly varied during transients. The key feature of the JFNK method is combining Newton's method to solve implicit nonlinear systems with Krylov subspace iterative methods. The Krylov methods do not require an explicit form of the Jacobian, which eliminates the computationally expensive step of forming Jacobian matrices (which also may be quite difficult to determine analytically), required by Newton's method. The matrix-vector product can be approximated by the numerical differentiation of nonlinear residual functions. Therefore, JFNK readily integrates different physics into one solver framework. 


\section{Model Description}

\subsection{Fluids Flow Models}

The RELAP-7 code has various flow models implemented. These include:

1). a single phase isothermal flow model (model_type=2),

2). a single phase nonisothermal fluid flow model (model_type=3),

3). a homogeneous equilibrium (HEM) two phase flow model (model_type=32),

4). a nonhomogeneous, nonequilibrium seven-equation two phase flow model (model_type=7).

\subsection{Equation of State}

For the single phase flow models, various types of equation of state can be used. These include:

1). Barotropic equation of state for isothermal flow. This is turned on by setting type=BarotropicEquationof State.

2). Linear equation of state for non-isothermal single phase flow. This is turned on by setting type = NonIsothermalEquationofState.

3). Stiffened gas equation of state which is turned on by setting type = StiffenedGasEquationofStateVapor for single phase vapor flow and type = StiffenedGasEquationofStateLiquid for single phase water flow.

4). Ideal gas equation of state which is turned on by setting type=IdealGasEquationof State. The equation of state for the nitrogen gas has also been implemented into the code. It can be turned on by setting type=N2Properties.

For the homogeneous equilibrium flow model, only the stiffened gas equation of state is applicable. This is turned on by setting type $=$ TwOPhaseStiffenedGasEOS.

For the seven-equation two phase flow model, only the stiffened gas equation of state is applicable. This is turned on by setting type = StiffenedGasEquationofStateLiquid for the liquid phase, and type = StiffenedGasEquationofStateVapor for the vapor phase. 


\subsection{Solution Stabilization Schemes}

It is well known that the continuous Galerkin finite element method is unstable when applied directly to hyperbolic systems of equations. Therefore the solution stabilization schemes are required for RELAP-7. Currently available options of solution stabilization for RELAP-7 include:

1). Streamline Upwind/Petrov Galerkin method (SUPG). The SUPG scheme works for the single phase flow only. It can be used by setting stabilization_type $=$ ' SUPG' .

2). Lapidus scheme works for both the single phase flow and the two phase flow cases. It can be used by setting stabilization_type = 'LAPIDUS' .

3). The entropy viscosity method works for both the single phase flow and the two phase flow. This option can be used by setting stabilization_type = 'ENTROPY_VISCOSITY'.

\subsection{Time Integration Schemes}

There are two types of time integration schemes in RELAP-7 - Implicit Euler and BDF2. Implicit Euler is a first order accurate time integration scheme. This can be turned on by setting:scheme = 'implicit-euler' in the Executioner input block (explained later). BDF2 is a second order accurate time integration scheme. This is the default option for RELAP-7.

\subsection{Components}

A real reactor system is very complex and contains hundreds of different physical components. It is impractical to resolve the real geometry of the entire system. Instead simplified thermal hydraulic models are used to represent (via "nodalization") the major physical components and describe the major physical processes (such as fluids flow and heat transfer). There are three main types of components developed in RELAP-7: (1) onedimensional (1-D) components describing the geometry of the reactor system, (2) zerodimensional (0-D) components for setting boundary conditions, and (3) 0-D components for connecting 1-D components. 


\subsubsection{Pipe}

Pipe is the most basic component in RELAP-7. It is a 1-D component which simulates thermal fluids flow in a pipe. Both a constant cross section area and a variable cross section area options are available for the Pipe component. The wall friction and heat transfer coefficients are either calculated through closure models or provided by user input. The pipe wall temperature can be provided as the wall heat transfer boundary condition. All the thermal fluids dynamic models are available in the Pipe component which includes the isothermal flow model, single-phase non-isothermal flow model, nonequilibrium 7equation two-phase model, and the much simpler homogeneous equilibrium two-phase flow model.

\subsubsection{PipeWithHeatStructure}

The PipeWithHeatStructure component simulates fluids flow in a 1-D pipe coupled with 1-D or 2-D heat conduction through the pipe wall. The adiabatic, Dirichlet, or convective boundary conditions at the outer surface of the pipe wall are available. Either a plate type or cylindrical type of heat structure can be selected. Volumetric heat source within the fluids or solid materials can be added.

\subsubsection{CoreChannel}

The CoreChannel component is a composite component designed to simulate the coolant flow and heat conduction inside a fuel rod as well as the conjugate heat transfer between the coolant and the fuel rod. In this component, the fuel rod is divided into the same number of segments as that of the coolant flow pipe elements. Each fuel rod segment is further simulated as 1-D or 2-D heat conduction model perpendicular to the fluid flow model. Both plate type fuel rod and cylindrical fuel rod type can be simulated. The solid fuel part is able to deal with typical LWR fuel rod with complex clad/gap/fuel pellet geometries. The flow model and conjugate heat transfer model are fully coupled in contrast to loosely coupled in RELAP5. 


\subsubsection{Subchannel}

A fully coupled subchannel channel model for the single-phase has been implemented into RELAP-7. The single-phase subchannel model includes four balance equations: mass, energy, axial momentum, and lateral momentum.

\subsubsection{HeatExchanger}

A Heat Exchanger component is a combination of two pipes with a solid wall in between. Similar to the CoreChannel model, the fluids flow model and conjugate heat transfer model are fully coupled. More complicated and realistic steam generator component will be developed in the future.

\subsubsection{Branch}

The branch model is a 0-D component representing a junction model with no volume (inertia) effects considered, and with single/multiple inlets and single/multiple outlets, of which cross section areas can be different. This model conserves the mass and energy among all connecting components.

\subsubsection{VolumeBranch}

The volume branch model is a 0-D component representing a joint/junction model with volume (inertia) effects considered. This model conserves the mass and energy among all connecting components.

\subsubsection{SubchannelBranch}

This is a OD branch model to connect the subchannel component. 


\subsubsection{Pump}

A simple pump model to provide a head and a reverse flow form loss coefficient $(K)$ for either isothermal flow and non-isothermal flow. It can be driven by an user input head or through a driving component which provides shaft work.

\subsubsection{Turbine}

The turbine model in RELAP-7 is a simplified dynamical turbine model to simulate a reactor core isolation cooling (RCIC) turbine, which drives the RCIC pump through a common shaft.

\subsubsection{SeparatorDryer}

The separator dryer model in RELAP-7 separates steam and water with mechanical methods. Only an ideal separator dryer model is available in RELAP-7.

\subsubsection{DownComer}

The down comer component simulates a large volume to mix different streams of water and steam and to track the water level.

\subsubsection{Valve}

The valve component simulates the open and close behaviors of valves for incompressible flow with user given trigger and response time. The abrupt area change model is used to calculate the form loss.

\subsubsection{CompressibleValve}

The compressible valve component simulates the open and close behavior of valves for compressible fluid flow, including ckoking. It can be used to simulate the safety relief 
valves (SRV) of BWRs.

\subsubsection{Check Valves}

The check valve component simulates the dynamic behavior of check valves, with the form loss calculated by the abrupt area change model.

\subsubsection{WetWell}

The wet well component simulates the dynamic response of a BWR suppression pool and its gas space.

\subsubsection{TimeDependentVolume}

The time depedent volume component provides pressure, temperature, and void fraction boundary conditions as constants or time dependent functions for 1-D components. It is a pure boundary condition type of component and it does not add any entries to the global unknown vector. When acquired by its connected 1-D component, it provides a pressure, a temperature, and a void fraction boundary condition.

\subsubsection{TimeDependentJunction}

The time dependent junction component provides velocity and temperature boundary conditions as constants or time functions for 1-D components.

\subsubsection{Tdm}

Tdm sets time dependent mass flow boundary conditions for 1-D components. 


\subsubsection{Reactor}

A virtual component that allows users to input the power for the core channel component.

\subsubsection{PointKinetics}

The point kinetics model is a lumped parameter neutron kinetics model to calculate the reactor power. User input reactivity or fully coupled feedback reactivity models are available. 


\section{Running RELAP-7}

\subsection{Complete Step 1 of MOOSE Environment Setup}

The system environment setup for MOOSE can be found with the link: http: //www. mooseframework .org/getting-started

\subsection{Setup Your SSH Key}

SSH key allows you to establish a secure connection between your computer and GitLab. Before generating an SSH key, check to see if your system already has one by running cat /.ssh/id_rsa.pub. If you see a long string starting with ssh-rsa or ssh-dsa, you can skip the ssh-keygen step below.

To generate a new SSH key, just open your terminal and use the code below. The ssh-keygen command prompts you for a location and filename to store the key pair and for a password. When prompted for the location and filename you can press enter to use the default. It is a best practice to use a password for an SSH key but it is not required and you can skip creating a password by pressing enter. Note that the password you choose here can not be altered or retrieved.

ssh-keygen -t rsa -c "\$your_email"

Use the code below to show your public key.

cat $\sim /$.ssh/id_rsa.pub

Copy and paste the key to the My SSH Key section under the SSH tab in your profile. Please copy the complete key starting with ssh-and ending with your username and host.

To test your SSH key.

\$ssh gitehpcgitlab.inl.gov

Welcome to GitLab, <your name here>!

Connection to hpcgitlab. inl.gov closed. 


\subsection{Checking Out the Code}

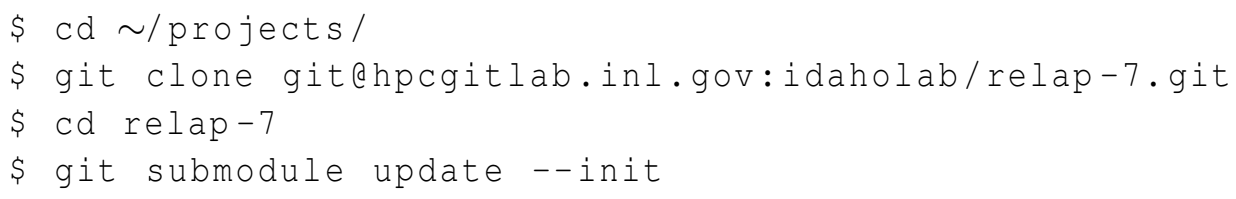

It is necessary to build libmesh before building any application.

\$ cd $\sim /$ projects/relap-7/moose

\$ . / update_and_rebuild_libmesh.sh

Once libmesh has been successfully compiled, you may now compile RELAP-7.

cd $\sim /$ projects/trunk/relap -7

make (add -jn to run on multiple "n" processors)

Once RELAP-7 has been compiled successfully, it is recommended to run the tests to make sure the version of the code you have is running correctly.

cd $\sim /$ projects/trunk/relap-7

. Irun_tests (add -jn to run "n" jobs at one time)

\subsection{Executing RELAP-7}

When first starting out with running RELAP-7, it is recommended to start from an example problem. Multiple example problems with input files are presented in this document. More examples can be found under the / relap-7/tests subdirectory. To demonstrate how to run RELAP-7, consider the PWR_core_channel. i test problem.

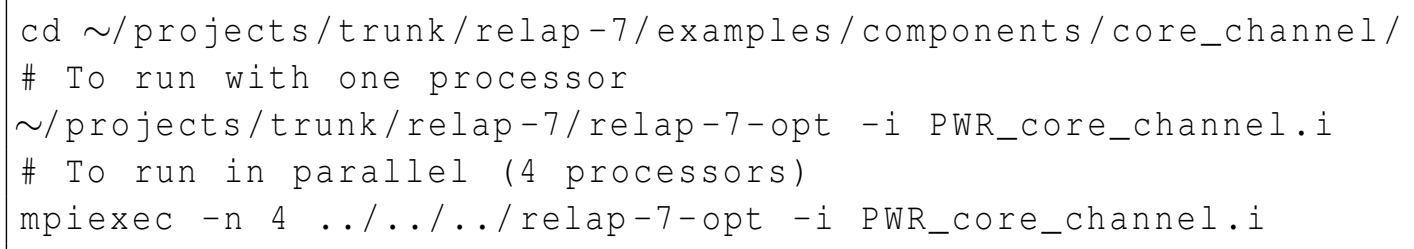




\subsection{Post Processing}

RELAP-7 typically writes solution data to an ExodusII file. The solution data may also be written in other formats, one being a comma separated values (CSV) file, which allows the solutionn data to be saved in a table structured format. The other being a tecplot file in either binary or ASCII format.

Several options exist for viewing ExodusII output files. One good choice is to use open-source software, Paraview (www. paraview.org).

\subsection{Graphical User Interface}

Another MOOSE based application named RAVEN provides a graphical user interface (GUI) for RELAP-7. RAVEN can be used to generate a text input fie. It is also capable of submitting the analysis and provides post processing capabilitis. 


\section{Input Files}

RELAP-7 uses a block-structured input file. Each block is identified with square brackets. The opening brackets contain the type of the input block and the empty brackets mark the end of the block. Each block may contain subblocks.

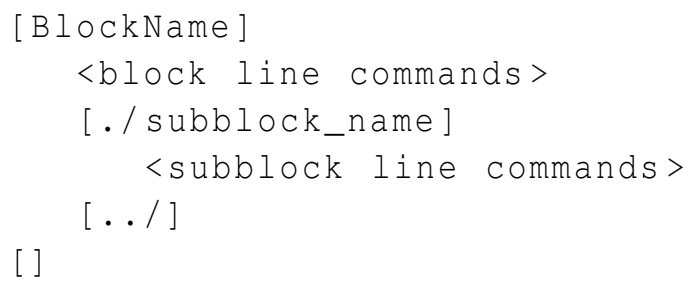

Each subblock must have an unique name when compared with all other subblocks in the current block.

Line commands are given as parameter and value pairs with an equal sign between them. They specify parameters to be used by the object being described. The parameter is a string, and the value may be a string, an integer, a real number, or a list of strings, integers, or real numbers. Lists are given in single quotes and are separated by whitespace.

Subblocks normally contain a type line command. This line command specifies the particular type of object being described.

RELAP-7 uses SI units. This stsndardizes the model input by eliminating the possibility of errors caused by using one set of units for one model and another set of units for a different model.

The following subsections have brief descriptions of each block. More detailed descriptions can be found in the examples section.

\subsection{Global Parameters}

The GlobalParams block specifies the global parameters used by the code such as the initial pressure (global_init_P), velocity (global_init_V) and temperature (global_init_T) of the system model, the fluid flow model type (model_type), the stabilzation scheme type (stabilization_type), and the scaling factors (scaling_factor_var) for the primary variable, etc. The values of of global parameters are available to any other block or sub- 
block in the input file. If a command line is missing in a block or a subblock but defined in GlobalParams, the block or subblock will use the parameter defined in GlobalParams. However, if the block or subblock has a command line, that will be used regardless of what is in GlobalParams.

The following is an example of the GlobalParams block:

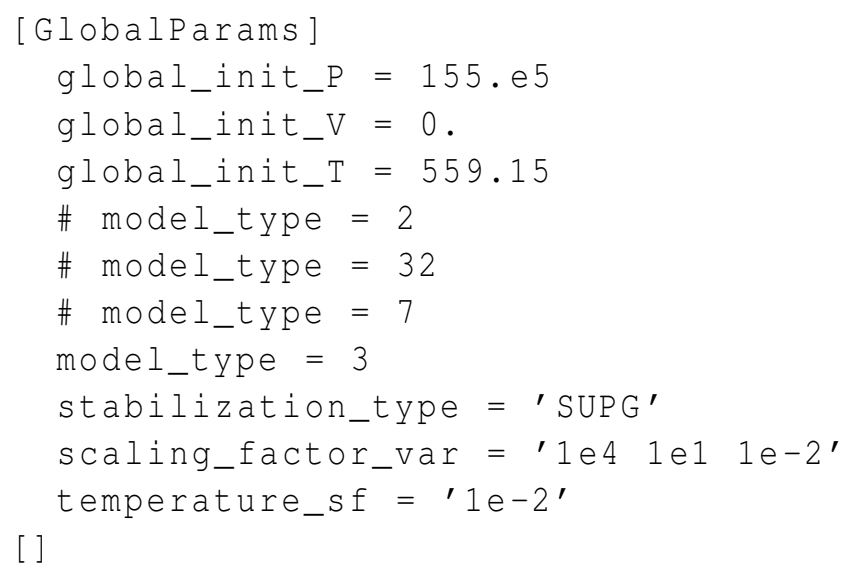

\# symbol indicates comments in the input file and can be located anywhere in the input file.

\subsection{Equation of State}

The EoS block specifies the equation of state to be used by the code. The following is an example of the EoS block:

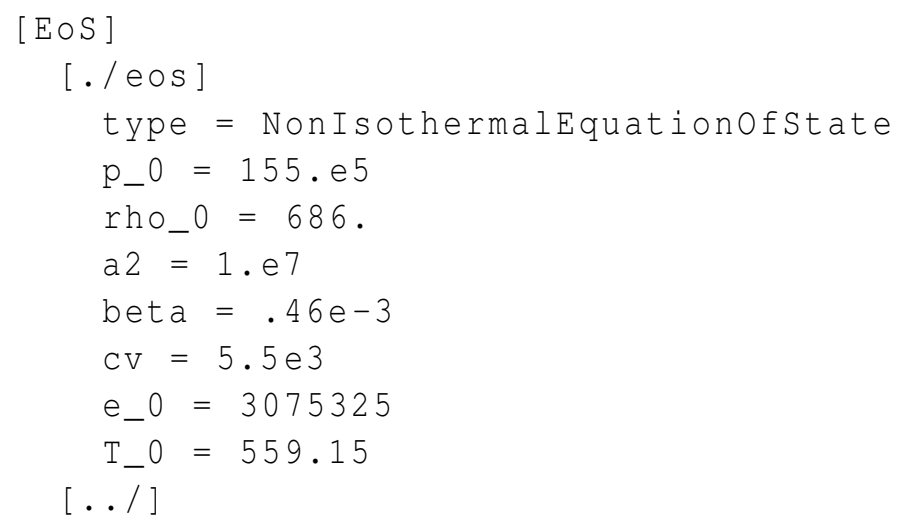




\subsection{Materials}

The Materials block specifies the properties of the solid materials for the code. The following is an example of the Materials block:

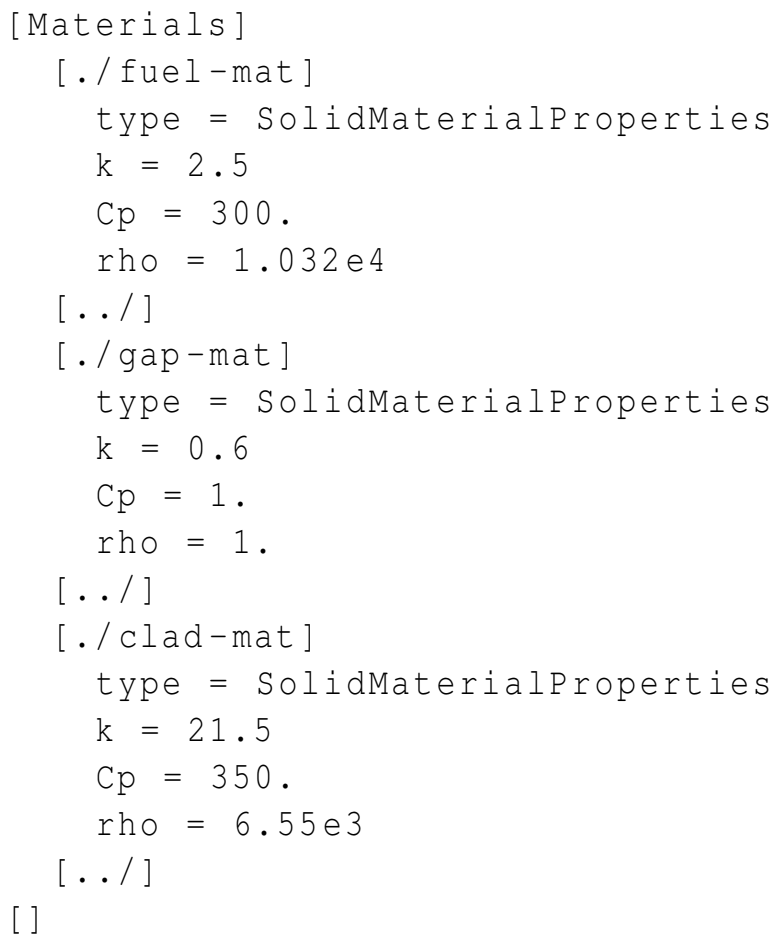

\subsection{Functions}

The Functions block provides the functions to be used by the code during the simulations such as reactor power as a function of time or a boundary condition pressure as a function of time, etc. The following is an example of defining pressure distribution as a function of $\mathrm{x}$.

[Functions ] 


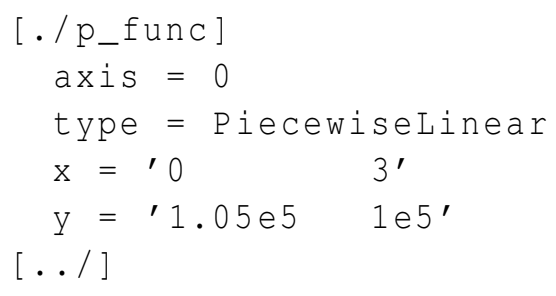

\subsection{Components}

The Components block specifies the components to be used in the simulations.

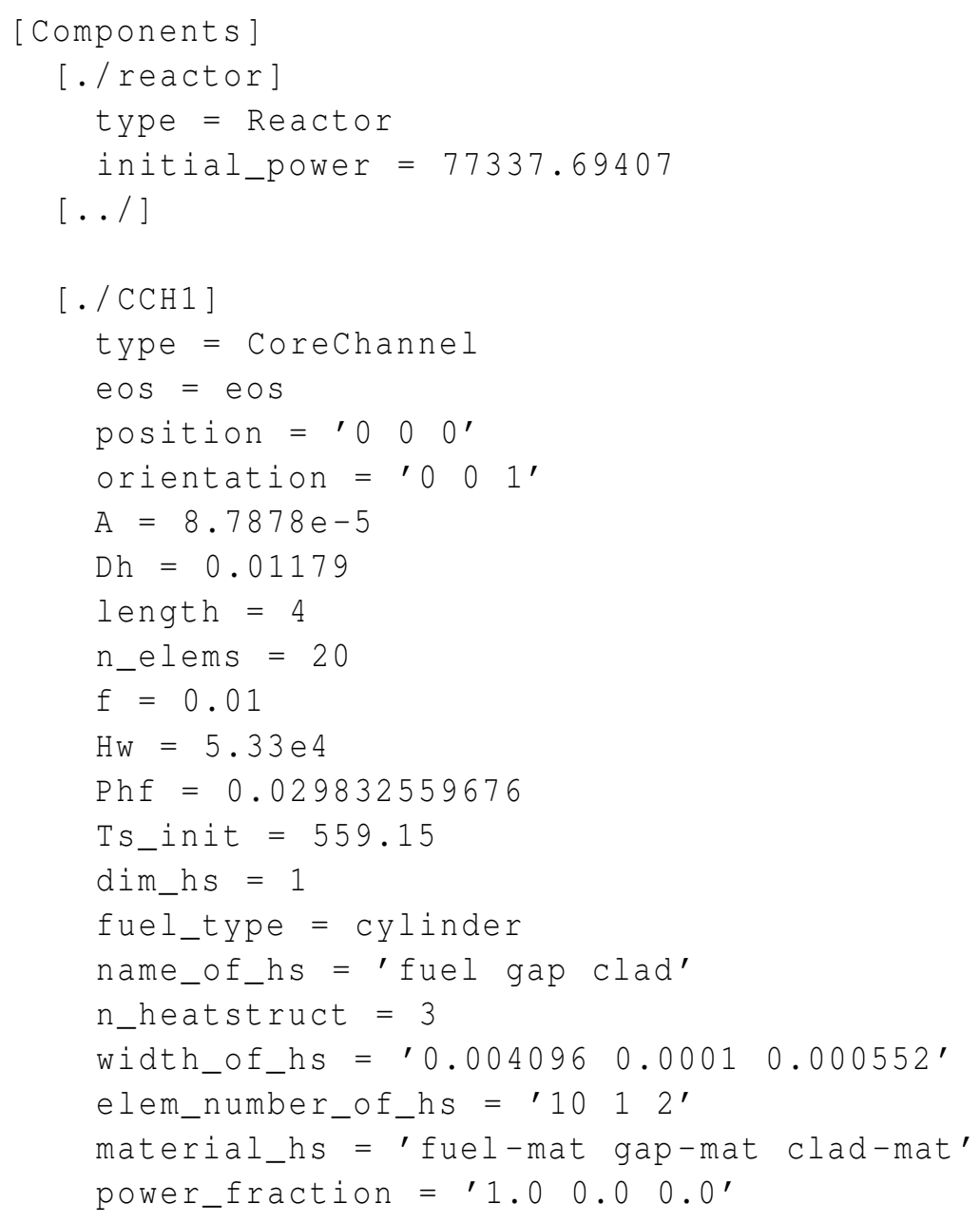




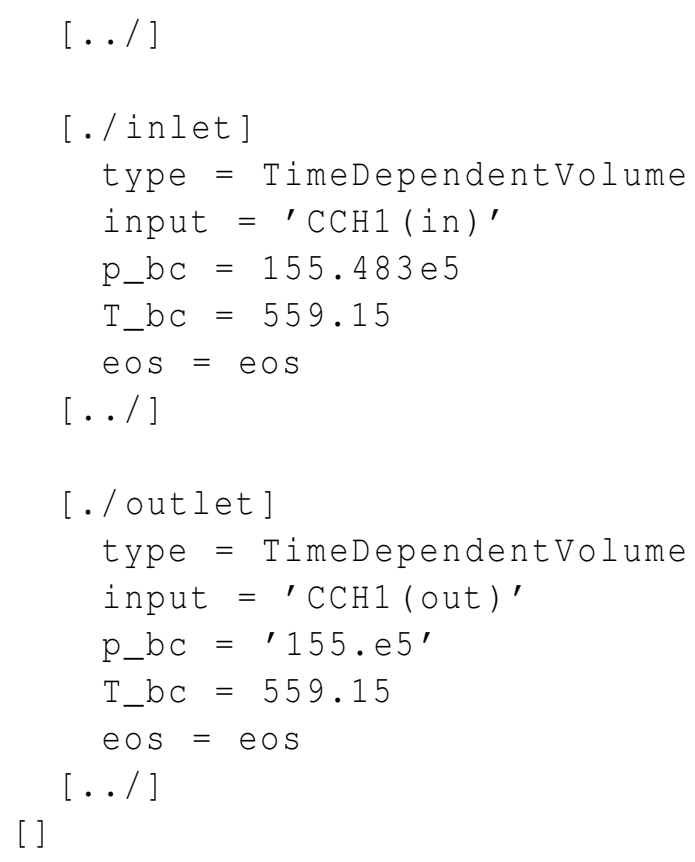

\subsection{Preconditioner}

The Preconditioning block specifies the preconditioner to be used by the preconditioned JFNK solver for the RELAP-7 code. The solution algorithm for RELAP-7 is the Jacobian-free Newton-Krylov (JFNK) method. However the Krylov methods need preconditioning to be efficient. Hence, the solvers available in RELAP-7 are preconditioned JFNK (PJFNK). Two options are available in RELAP-7 to build the preconditioning matrix, the single matrix preconditioner (SMP) and the finite difference preconditioner (FDP). The SMP option uses all the Jacobian terms derived analytically to build one preconditioning matrix. The FDP option uses numerical Jacobian by doing direct finite differences of the residual terms. The SMP option is the more efficient and is the recommended option, while the FDP option is normally slow and inefficient and is recommended to be used for small problems or for debugging purposes. The following is an example of the Preconditioning block:

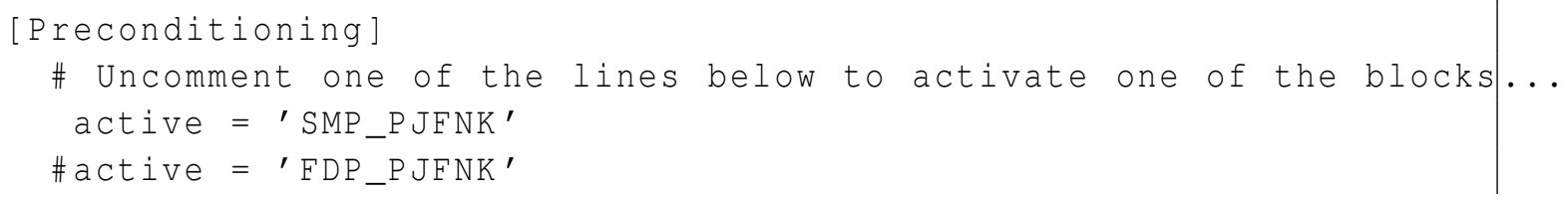




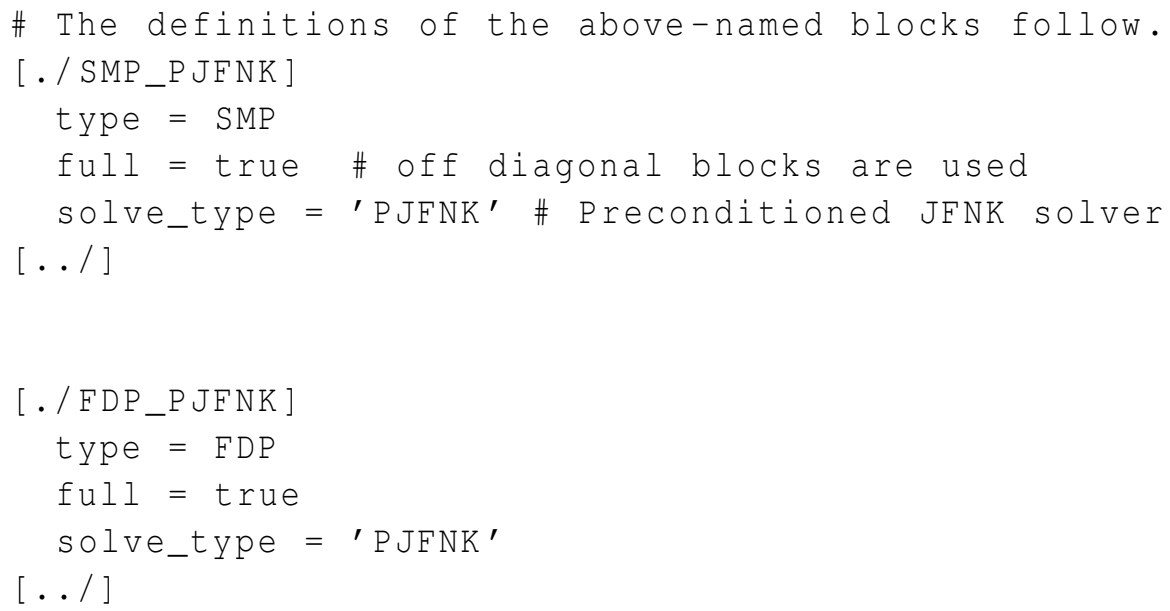

\subsection{Executioner}

The Executioner block specifies the executioner that will be used in the simulations. The options include Transient, RavenExecutioner, etc.

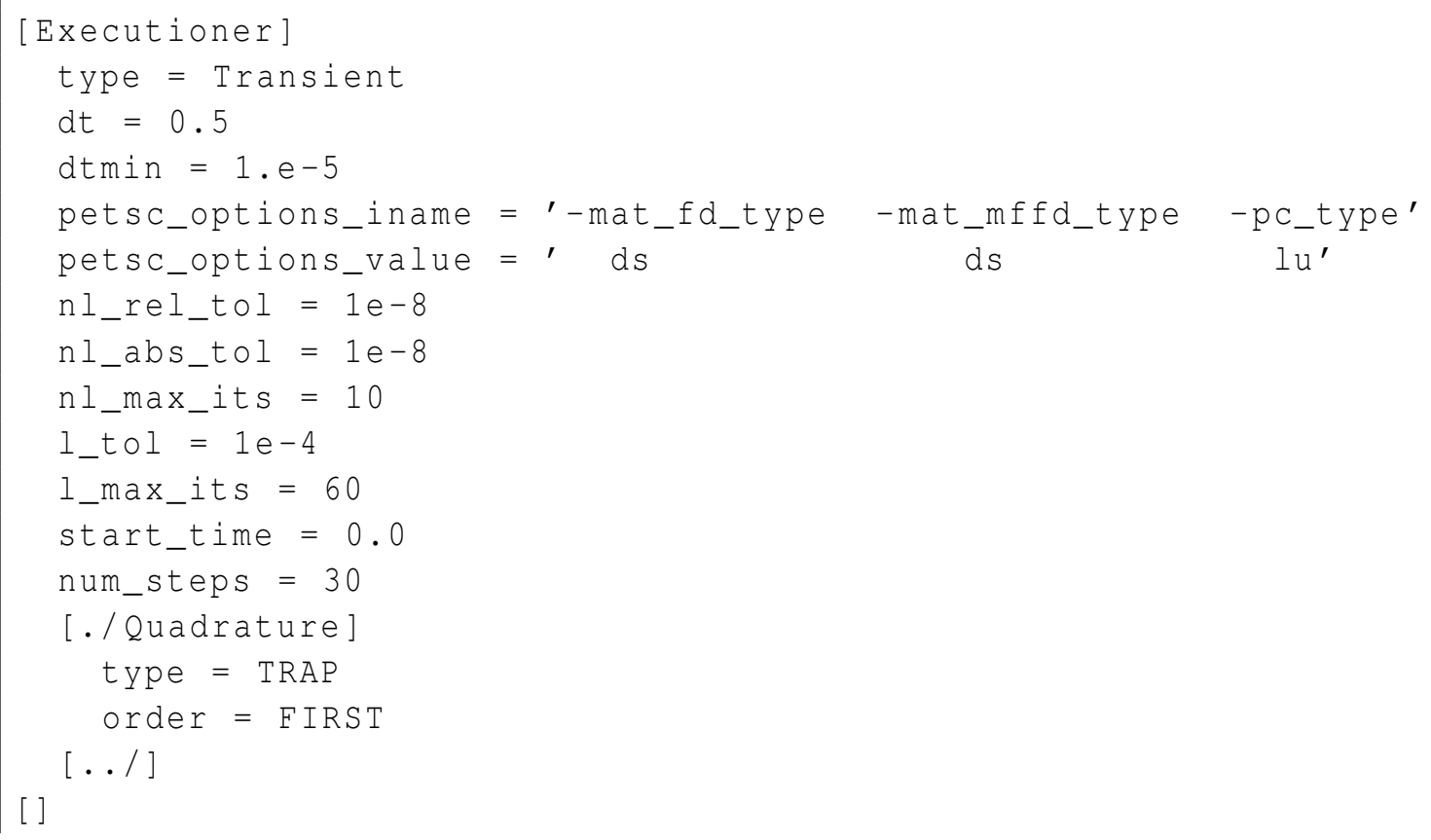




\subsection{Outputs}

The Outputs block controls the various screen and file output in the simulations.

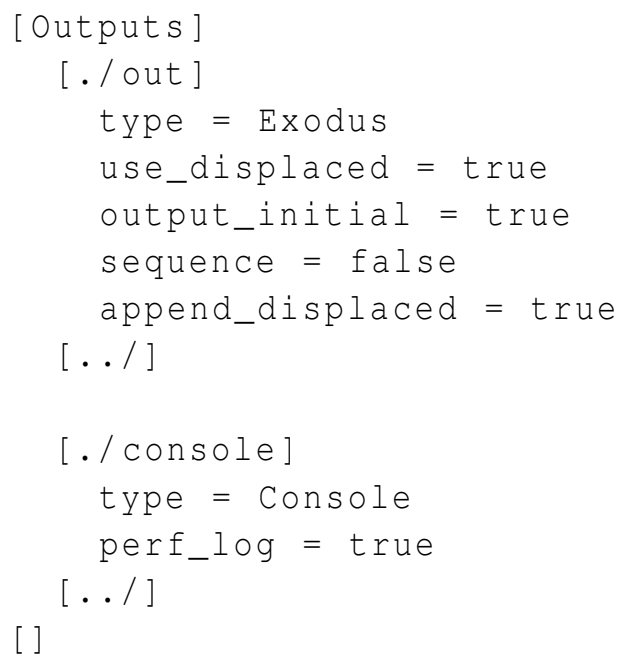




\section{Examples}

\subsection{Example 1: A Simple Pipe Flow Problem}

\subsubsection{Problem Description}

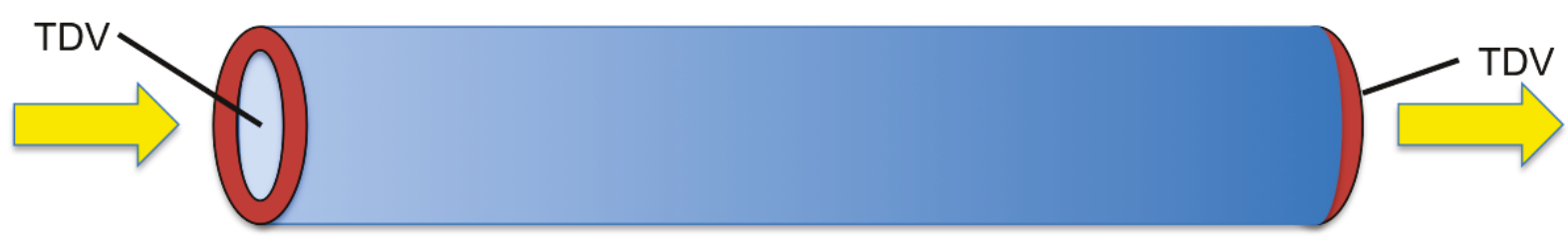

Figure 1. A simple pipe flow problem diagram

Example 1 simulates water flowing through a pipe under isothermal conditions with the following parameters:

Hydraulic Diameter $=.01 \mathrm{~m}$

Cross section flow area $=7.85 \mathrm{e}-5 \mathrm{~m}^{2}$

Length $=1 \mathrm{~m}$

Wall friction coefficient $=.01$

The boundary conditions are the following:

Inlet: Pressure $=1.05 \mathrm{e} 5 \mathrm{~Pa}$

Outlet: Pressure $=1 \mathrm{e} 5 \mathrm{~Pa}$

A 1D model can be viewed in Paraview to visualize the process better. Once Paraview is opened, on the left, select all of the variables and click "Apply." In the filters tab, under "Data Analysis", select "Plot Over Line" and apply. When this is done, each parameter in the problem can be viewed plotted over the length of the pipe. The figure below shows pressure vs length. 


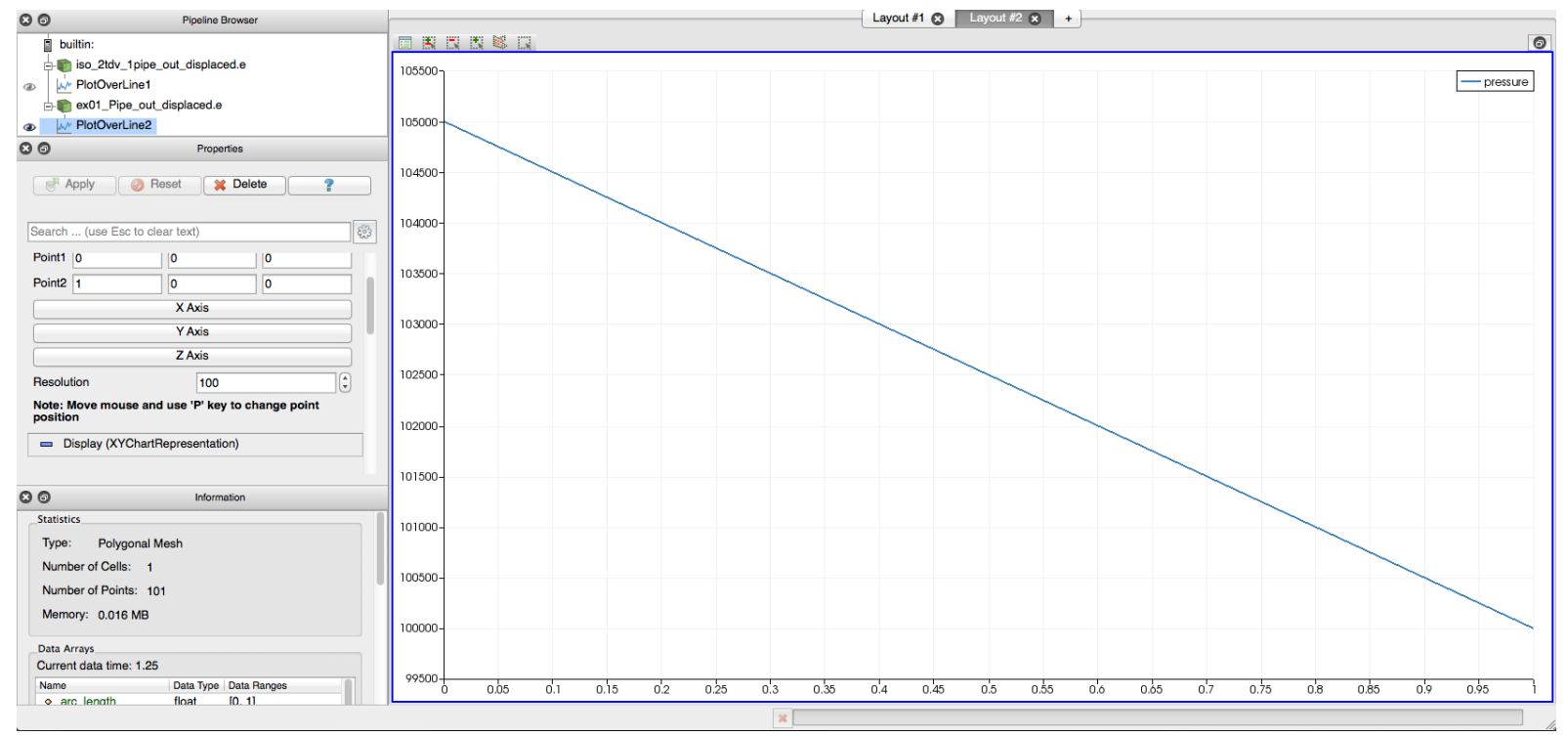

Figure 2. Pressure vs. length for the simple pipe flow problem

The pressure difference can be hand calculated to check the code calculated results using:

$$
\Delta P=\frac{f L \rho u^{2}}{2 D_{h}}
$$

Where $\rho$ is the density and $u$ is the velocity. Both values can be found in Paraview to perform the calculation.

\subsubsection{Input File}

The following shows the input file to run this example problem: 


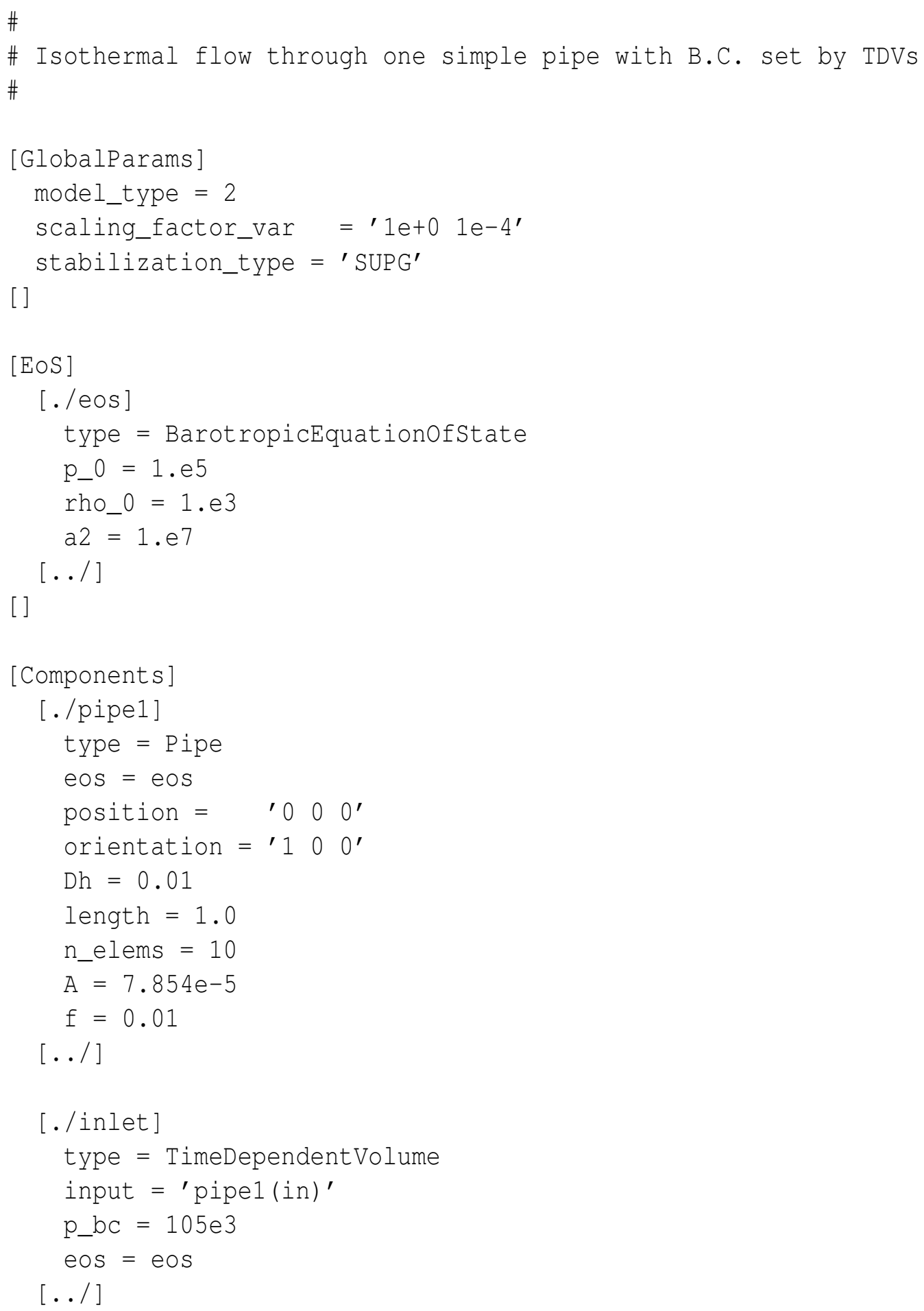




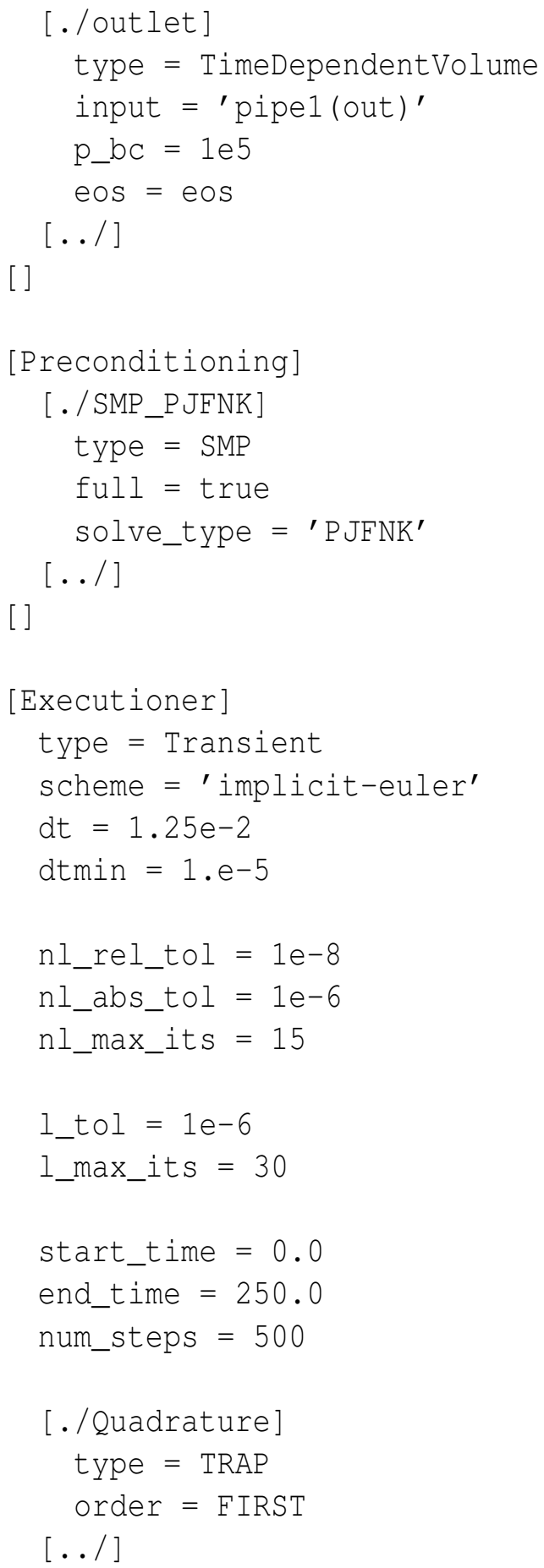




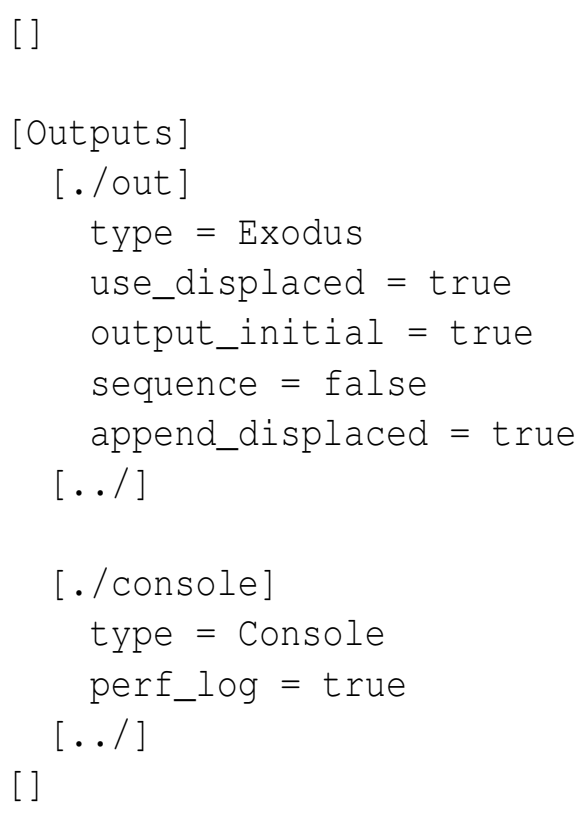

\subsubsection{Description of the Input File}

The following are the destailed descriptions of the input file block by block.

\section{The Global Parameters block:}

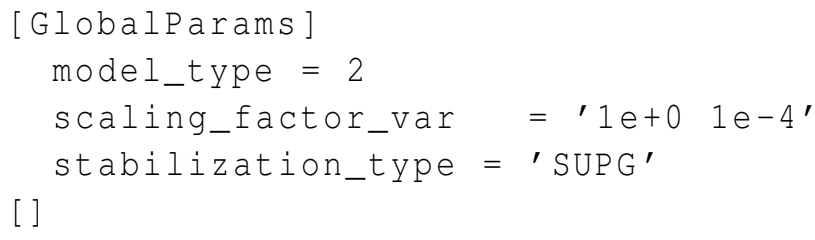

model_type $\quad$ Fluid flow model. 2 means single phase isothermal fluid flow.

scaling_factor_var Scaling factor for the primary variables $\rho A$ and $\rho u A$ respectively.

stabilization_type Stabilization scheme type. Available optionsd are: SUPG for single phase flow, LAPIDUS for both single- and two-phase flow, ENTROPY_VISCOSITY for both single- and two-hase flow. 
The Equation of State block:

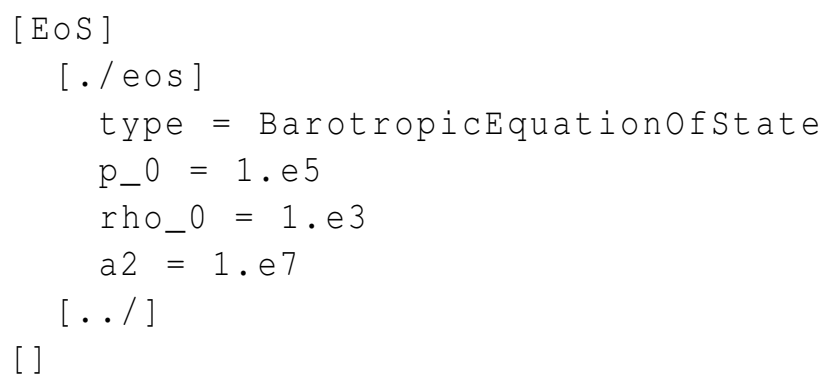

\section{[./eos] Subblock for eos}

type The type of equation of state to be used. The barotropic equation of state is suitable for isothermal fluid flow model. It describes isentropic (reversible) processes, and implies a constant sound speed. It is given by $p=p_{0}+a^{2}(\rho-$ $\left.\rho_{0}\right)$.

p_0 Initial pressure value $(P a)$ in the equation of state calculations.

rho_0 Initial value of density $\left(\mathrm{kg} / \mathrm{m}^{3}\right)$ in the equation of state calculation.

a2 $a$ is a constant in the barotropic equation of state. The value of $a$ can be taken roughly as the speed of sound. For this example problem with water as the fluids, using $a^{2}=1 . e 7$ has the same order of magnitude as that of the square of sound speed. This is the recommended value to use.

\section{The Components Block:}

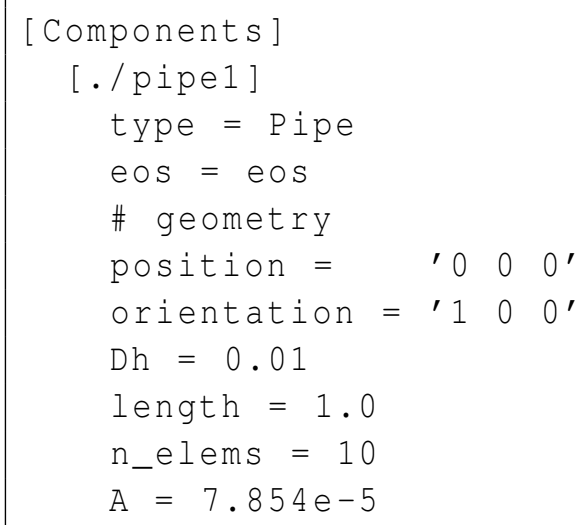




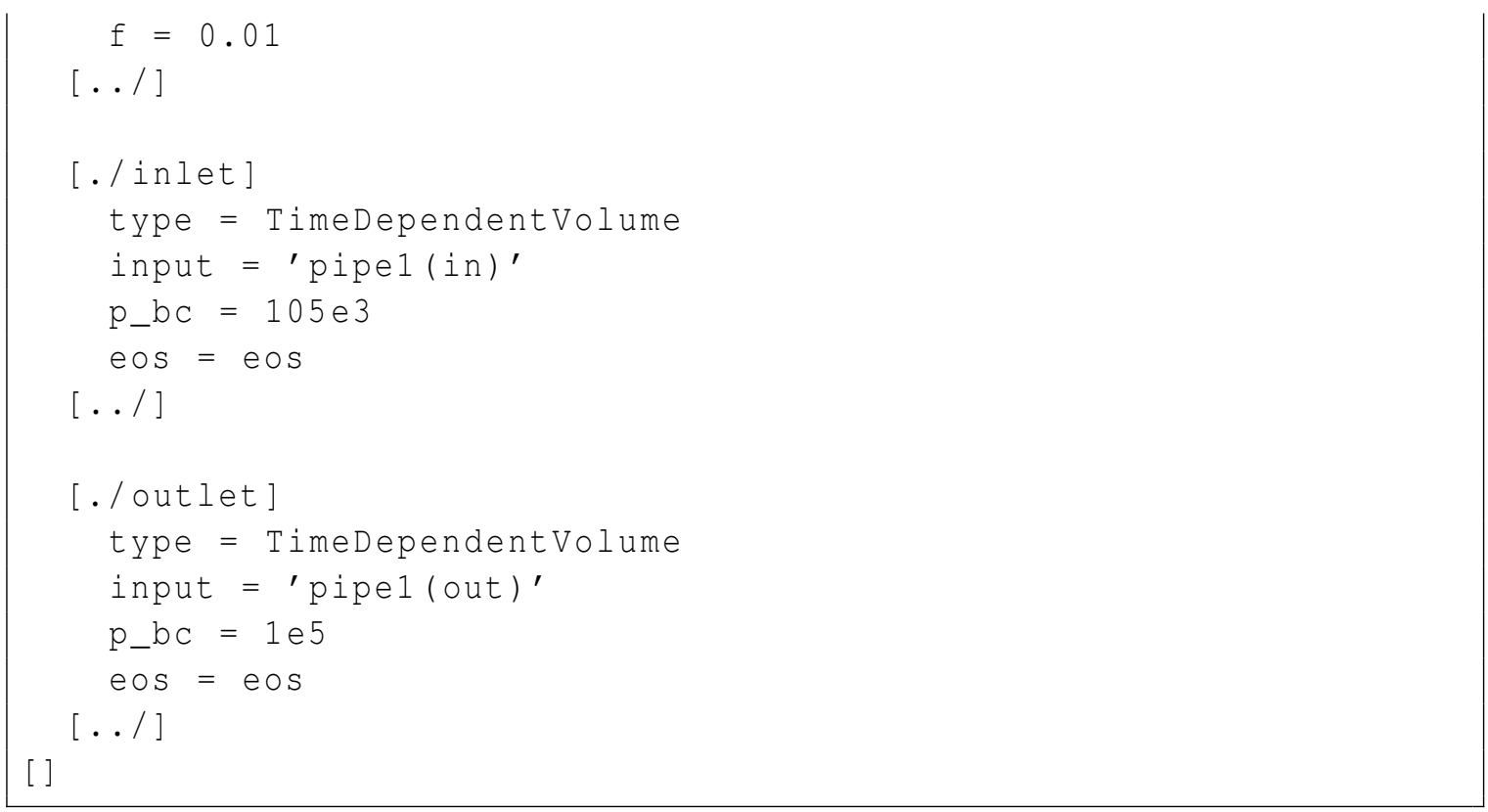

[./pipe1] Subblock for pipe1.

type Component type is pipe.

eos Use the eos equation of state defined in the EoS block.

position The starting $\mathrm{x}, \mathrm{y}$ and $\mathrm{z}$ coordinate of pipe1.

orientation The orientation vector of pipe1. Horizontal pipe for this case.

Dh Hydraulic diameter of of the pipe.

length Pipe length.

n_elems Number of elements in the solution of the pipe.

A The cross section flow area of the pipe.

f The wall friction coefficient. If this is not provided in the input file, the code built-in correlations will be used to calculate the friction coefficients (for single phase flow only). 
[./inlet $\quad$ Subblock for the component to set the inlet boundary conditions.

type $\quad$ Component type is time dependent volume.

input The pipe inlet end which will be connected with this component.

p_bc The pressure boundary condition value at the inlet of the pipe.

eos The equation of state to be used.

[./ outlet] Subblock for the component to set the outlet boundary conditions.

type $\quad$ Component type is time dependent volume.

input The pipe outlet end which will be connected with this component.

p_bc The pressure boundary boundary condition value at the outlet of the pipe.

eos The equation of state to be used.

\section{The Preconditioning Block:}

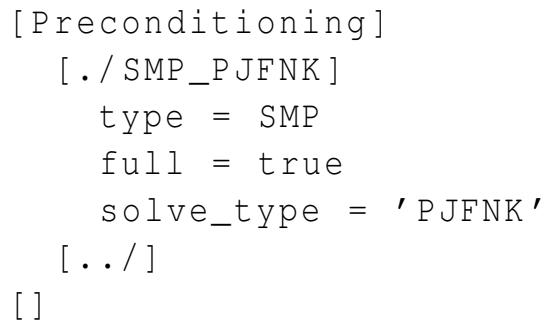

[ . /SMP_P JFNK] Subblock for SMP preconditioner and PJFNK solver. This is the recommended user opton. For advanced users, please refer to MOOSE and PETSc manuals.

type $\quad$ Indicating the preconditioning matrix type is SMP for this case.

full true means the entire preconditioning matrix will be assembled in the calculations. 
solve_type PJFNK solver will be used for this problem.

\section{The Executioner Block:}

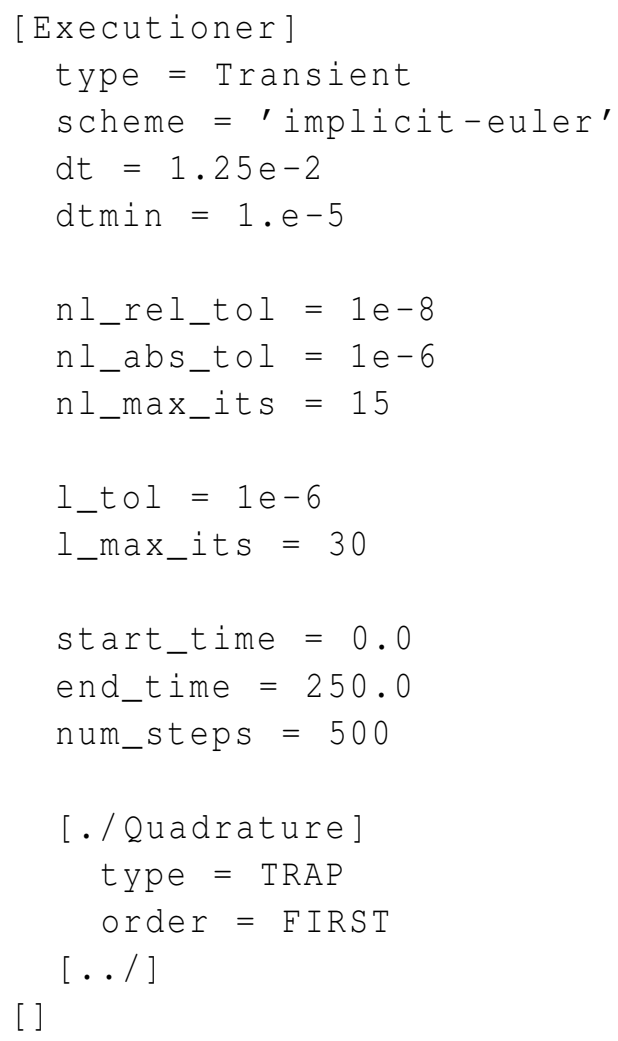
default option of BDF2 will be used.

$d t$ Time step size to be used .

dtmin Minimum time step size to be used. If the time step size is below this value, the case will stop executing.

nl_rel_tol Relative tolerance for the nonlinear solve. The recommended value is $1.0 \mathrm{E}-8$. 
nl_abs_tol

nl_max_its

l_tol

l_max_its

start_time

end_time

num_steps

type

order
Absolute tolerance for the nonlinear solve. The recommended value is $1.0 \mathrm{E}-6$.

Maximum number of nonlinear solve iterations. The recommended value is 15 .

Relative tolerance for the linear Krylov solve. The recommended value is $1.0 \mathrm{E}-6$.

Maximum number of liner iterations. The recommended value is 30.

The start time of the simulation.

The end time of the simulation.

The maximum number of time integration steps for the simulation. The simulation will stop either the maximum number of time steps is reached or the end_time is reach.

The quadrature subblock. This is the recommended option.

Type of the quadrature rule. Advanced users can refer to the MOOSE manual for other options.

Order of the quadrature.

\section{The Outputs Block:}

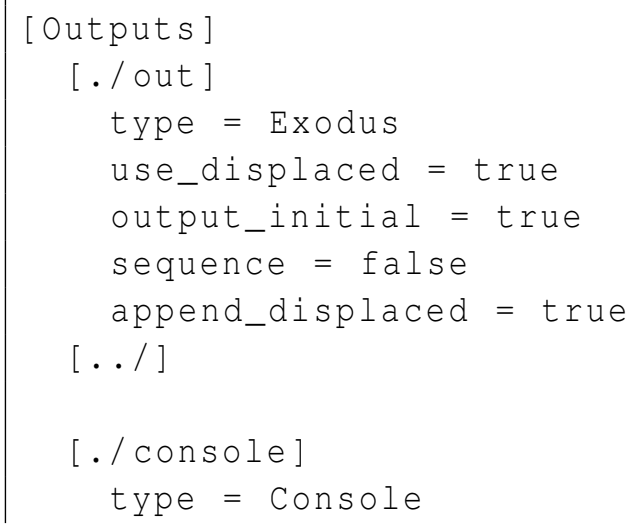




\begin{tabular}{|c|c|}
\hline$[. /$ out $]$ & Output subblock. This is the recommended option. \\
\hline type & Exodus output file type. \\
\hline use_displaced & Use the displaced mesh to show the results. \\
\hline output_initial & The initial condition is output to the solution file. \\
\hline sequence & Enable or disable sequential file output. \\
\hline append_displaced & Append '_displaced' to the output file name. \\
\hline$[. /$ console $]$ & Console subblock. \\
\hline type & Console type. \\
\hline perf_log & Print all performance logs. \\
\hline
\end{tabular}




\subsection{Example 2: Use Functions to Set Initial Conditions for a Simple Pipe Flow Problem}

\subsubsection{Problem Description}

This example illustrates how to use functions to set the initial conditions. The example used here is to set the initial pressure distribution in a pipe for an isothermal flow through one simple pipe with boundary conditions set by time dependent volumes (TDV).

Functions:

Initial pressure distribution in the pipe Axis $=0$ The axis used $(0,1$, or 2 for $\mathrm{x}, \mathrm{y}$, or $\mathrm{z})$ if this is to be a function of position. Note that for RELAP-7 1-D component such as a pipe, we only use $x$ coordinate in the calculations therefore we only need to set Axis $=0$.

Type $=$ PiecewiseLinear

$\mathrm{x}=$ '0 3' This is the pipe axial direction spatial coordinate relative to the starting point $\mathrm{y}={ }^{\prime} 1.05 \mathrm{e} 51 \mathrm{e}{ }^{\prime}$

Initial velocity distribution in the pipe

Axis $=0$

Type $=$ PiecewiseLinear

$\mathrm{x}={ }^{\prime} 03^{\prime}$

$\mathrm{y}={ }^{\prime} 33^{\prime}$

Fig. 3 shows the plot of pressure versus pipe length for this case. 


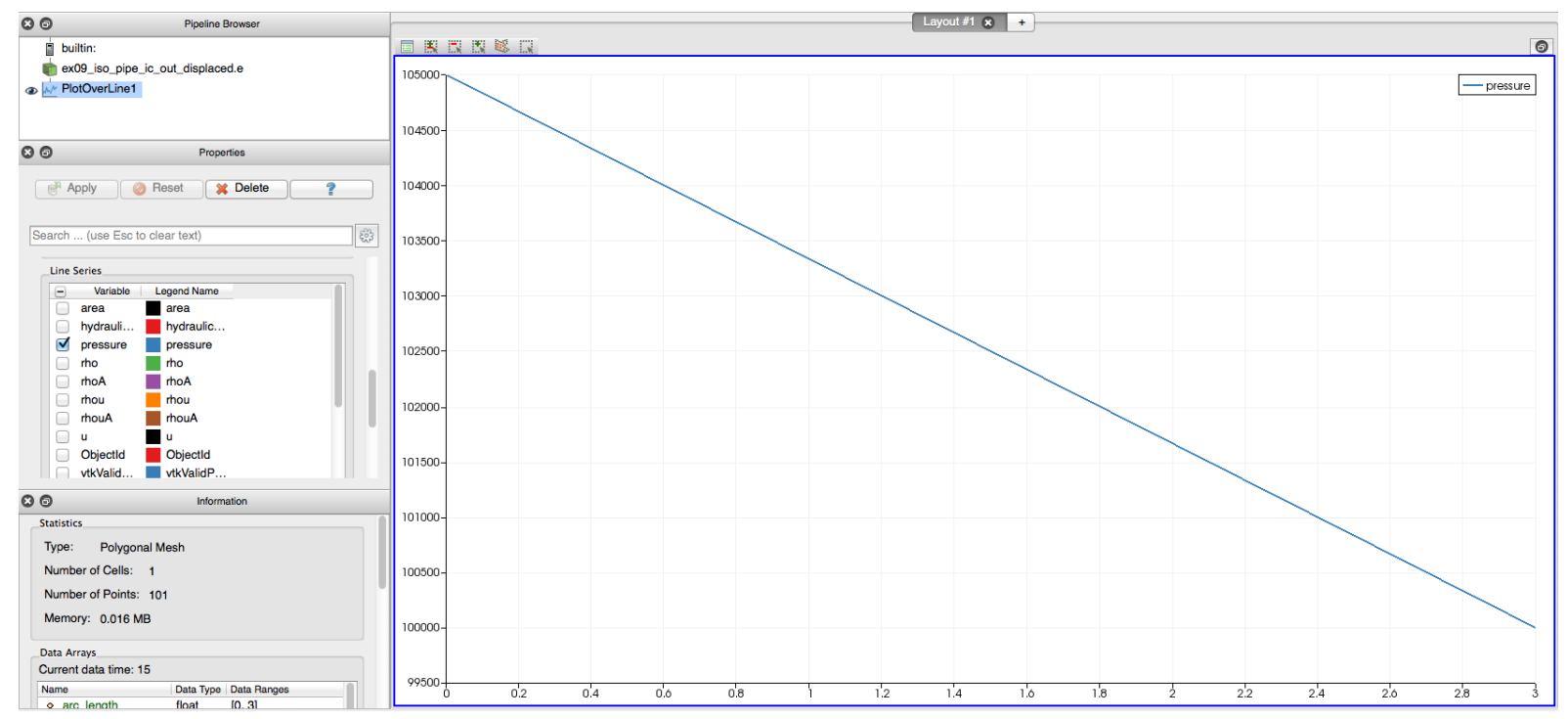

Figure 3. Pressure vs length for an isothermal pipe flow problem with initial pressure set by functions

\subsubsection{Input File}

The input file for this example is shown as follows:

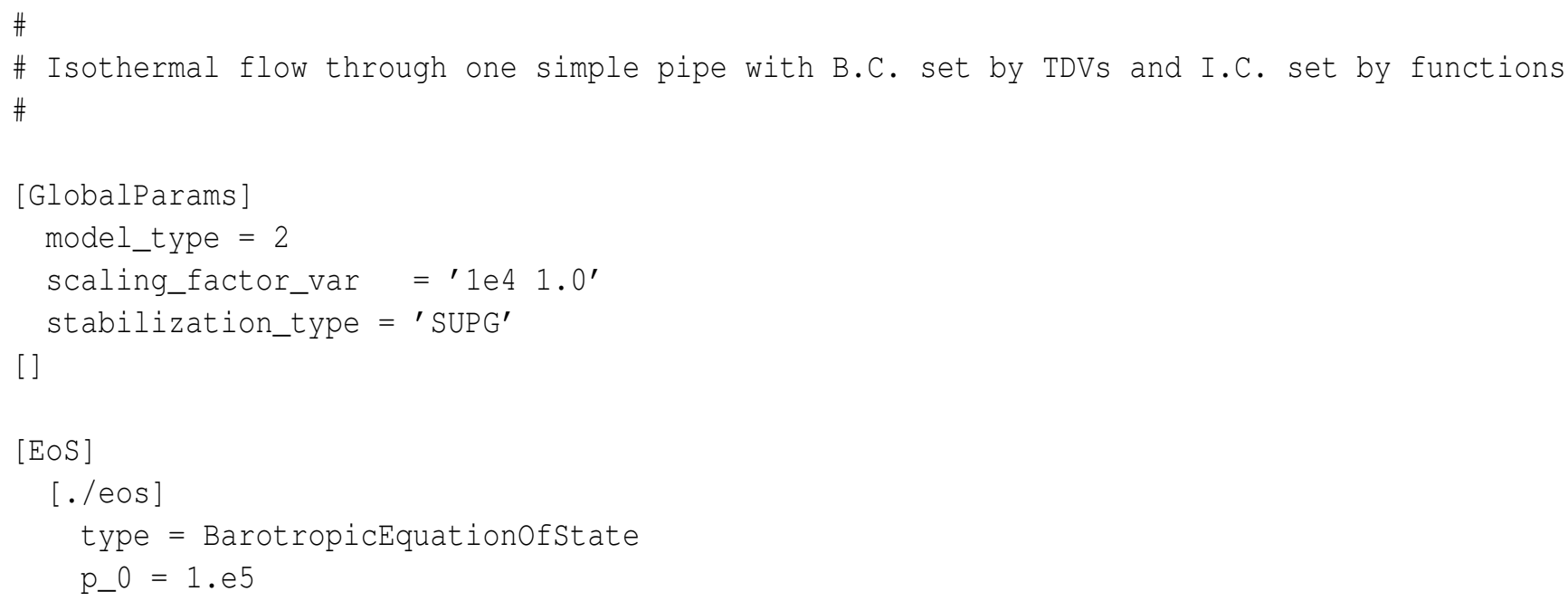




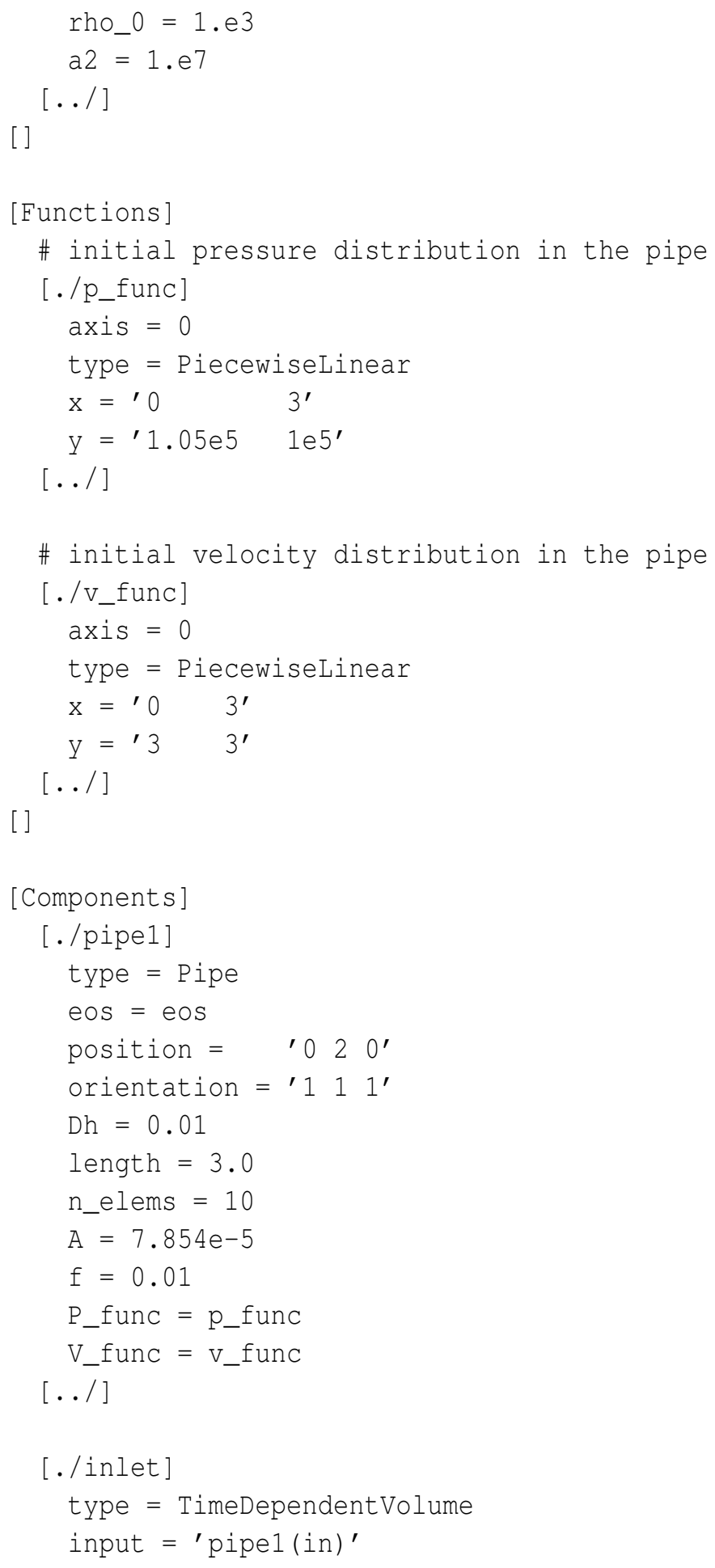




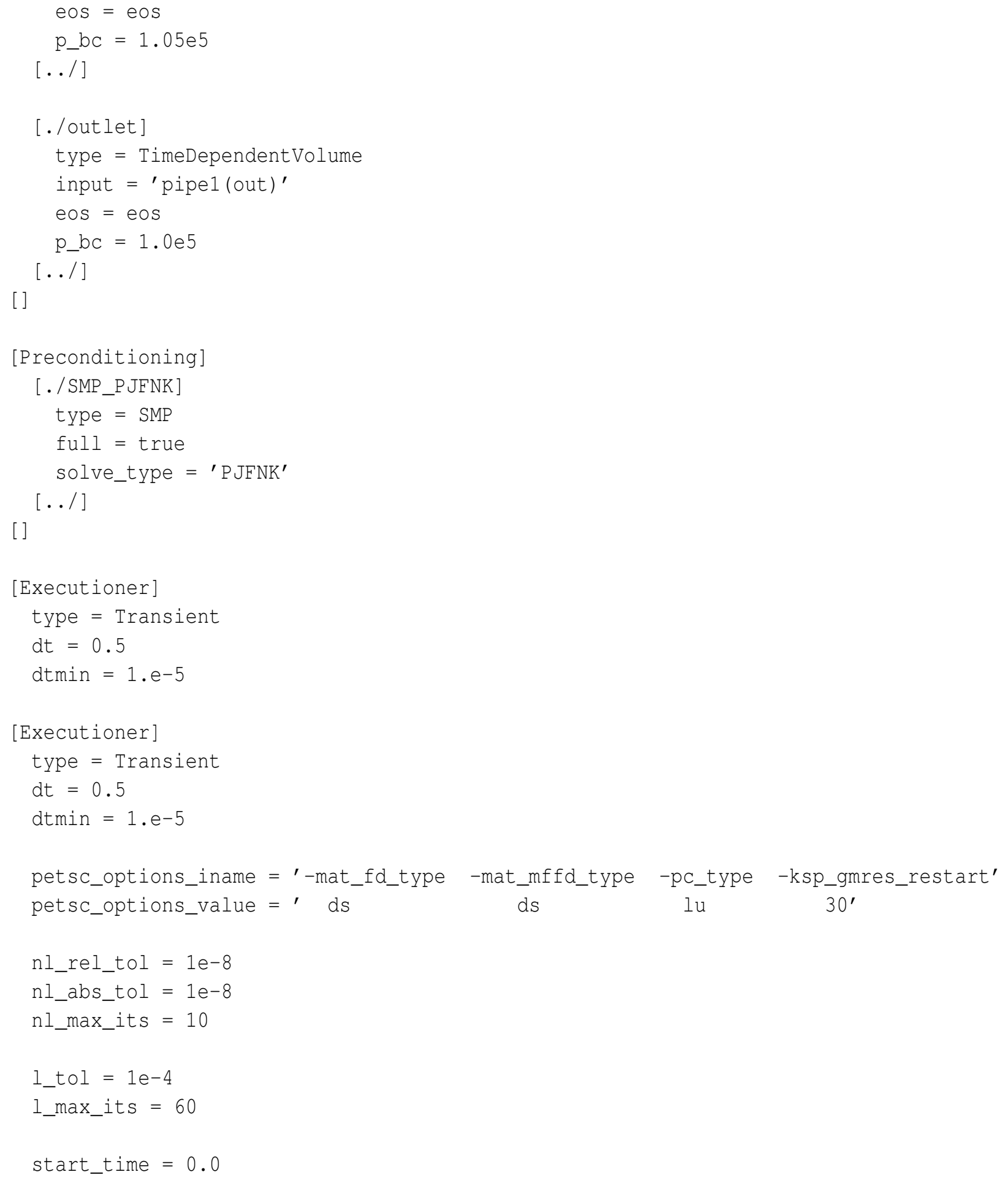




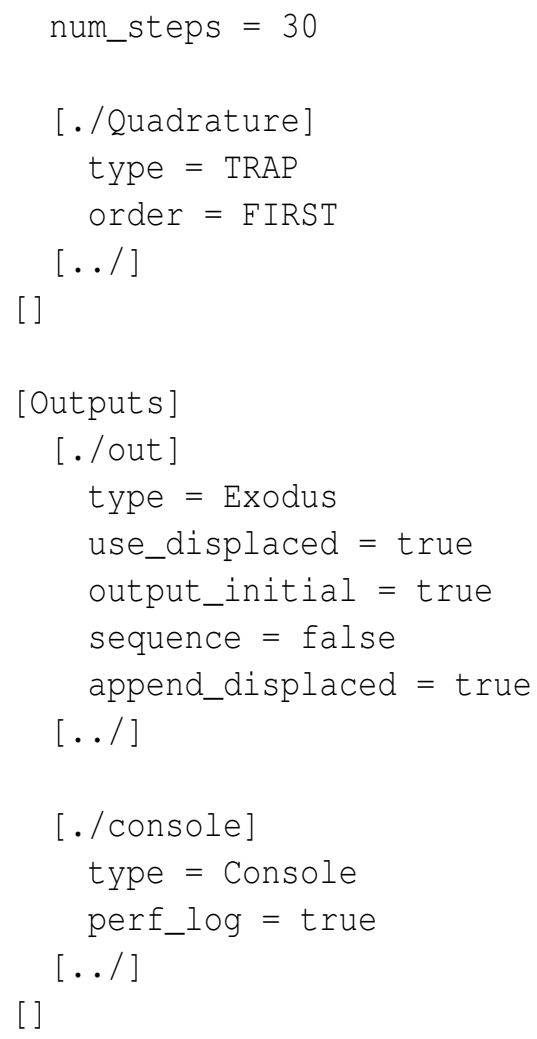

\subsubsection{Description of the Input File}

The following will provde explanations to the input parameters that did not exist in Example 1.

\section{The Functions Block:}

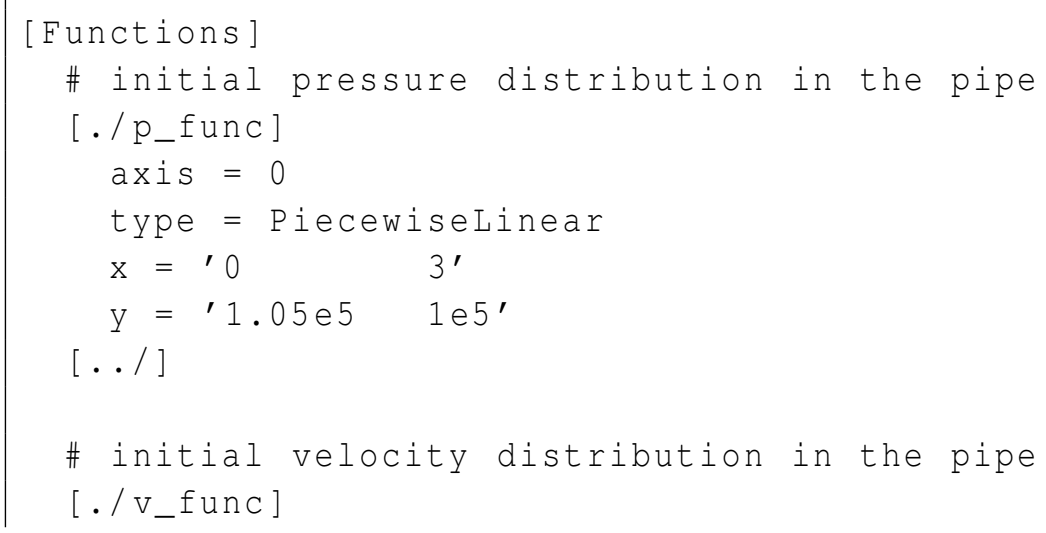




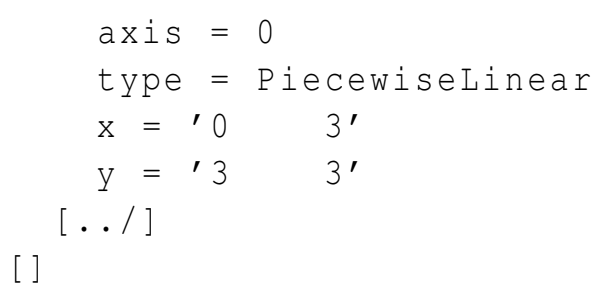

[./p_func $]$ Subblock for the pressure distribution function.

axis $\quad$ For RELAP-7 1-D components, only the $\mathrm{x}$ coordinate is used in the calculations and hence axis is set to be 0 which allows a function to be defined as a function $x$. If axis is not given in the input file, the data pair of $(x, y)$ will be taken as a time function.

type Type of functions used to interpolate data between data points.

$\mathrm{x}$ The coordinate used for the $\mathrm{x}$-axis data.

Y The initial pressure distribution function data point values.

The Components Block:

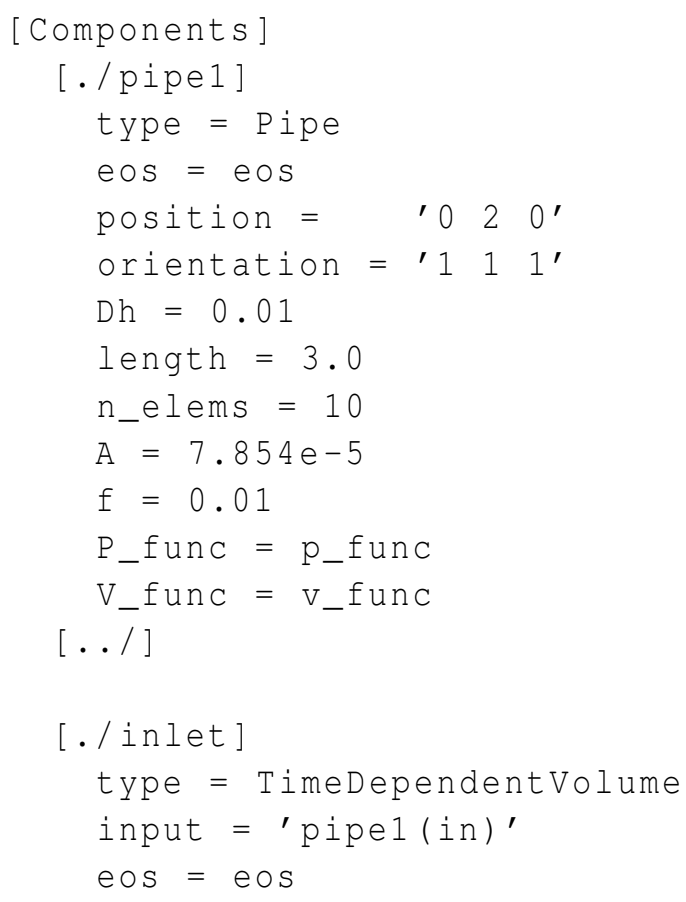




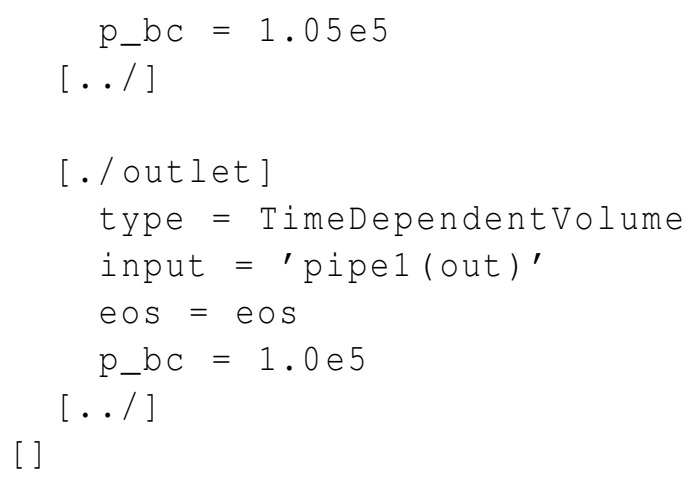

P_func Use the pressure function defined in the Functions block to set the initial pressure value.

V_func Use the velocity function defined in the Functions block to set the initial velocity value.

\section{The Executioner Block:}

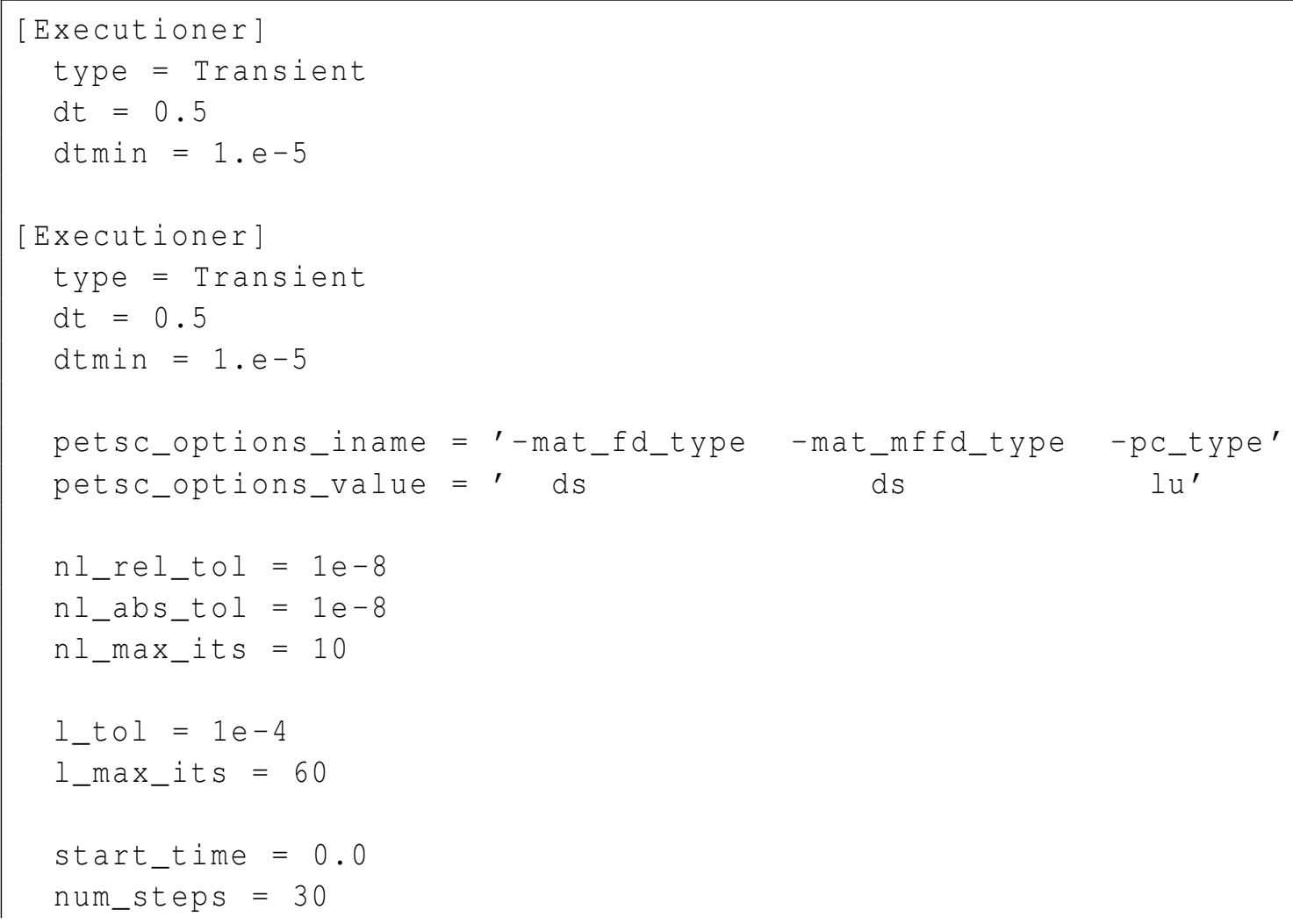




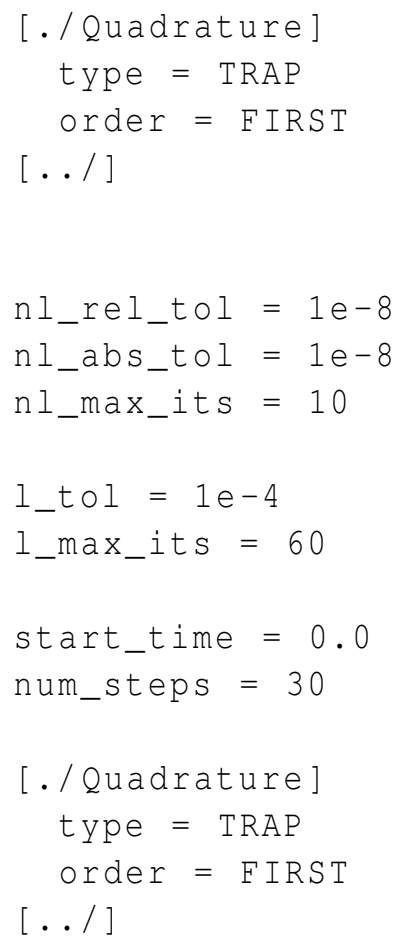

petsc_options_iname Names of PETSc name and value pairs. -mat_fd_type $=d s$ is an option to control how the Jacobian matrix is approximated by using the finite differencing method. -mat_mffd_type $=d_{s}$ is an option to control how matrix-free operation is approximated by using the finite difference method. Users are recommended to refer to the PETSc manual for other options.

petsc_options_value Values of PETSc name/value paris. 


\subsection{Example 3: Two Phase Flow Through a Pipe - HEM Model}

\subsubsection{Problem Description}

Two phase flow with HEM model through one simple pipe with boundary conditions set by time dependent volumes and initial conditions set by functions. The initial conditions are close to steady state solutions; therefore the simulation quickly converges to the steady state.

The following is the description of the functions defined in the Functions block:

Initial pressure distribution in the pipe

Axis $=0$

Type $=$ PiecewiseLinear

$\mathrm{x}={ }^{\prime} 01$ '

$y={ }^{\prime} 7.01 \mathrm{e} 67 \mathrm{e} 6 '$

Initial velocity distribution in the pipe

Axis $=0$

Type $=$ PiecewiseLinear

$\mathrm{x}=$ '0 1'

$\mathrm{y}={ }^{\prime} 1.352 .1{ }^{\prime}$

Initial temperature distribution in the pipe

Axis $=0$

Type $=$ PiecewiseLinear

$\mathrm{x}=$ '0 0.041 '

$y=' 517517.343517 .253$ '

Initial void fraction distribution in the pipe

axis $=0$

type $=$ PiecewiseLinear

$\mathrm{x}={ }^{\prime} 00.041$ '

$\mathrm{y}={ }^{\prime} 00.0 .37$ '

The parameters and functions used for the pipe component are the following:

Position = ' 000 O'

Orientation $=$ '1 100 , 


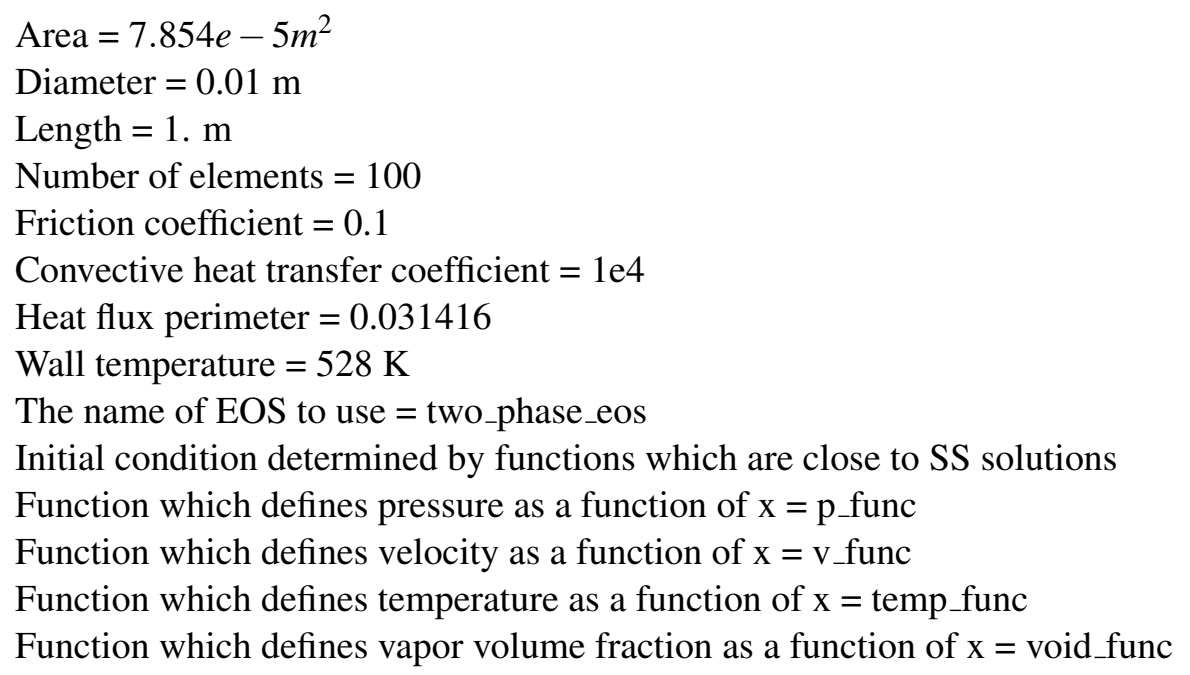

The inlet boundary conditions:

Vapor volume fraction $=0$.

The name of EOS to use $=$ two_phase_eos

The outlet boundary conditions:

Vapor volume fraction $=0.5$

The name of EOS to use $=$ two_phase $\_$eos 


\subsubsection{Input File}

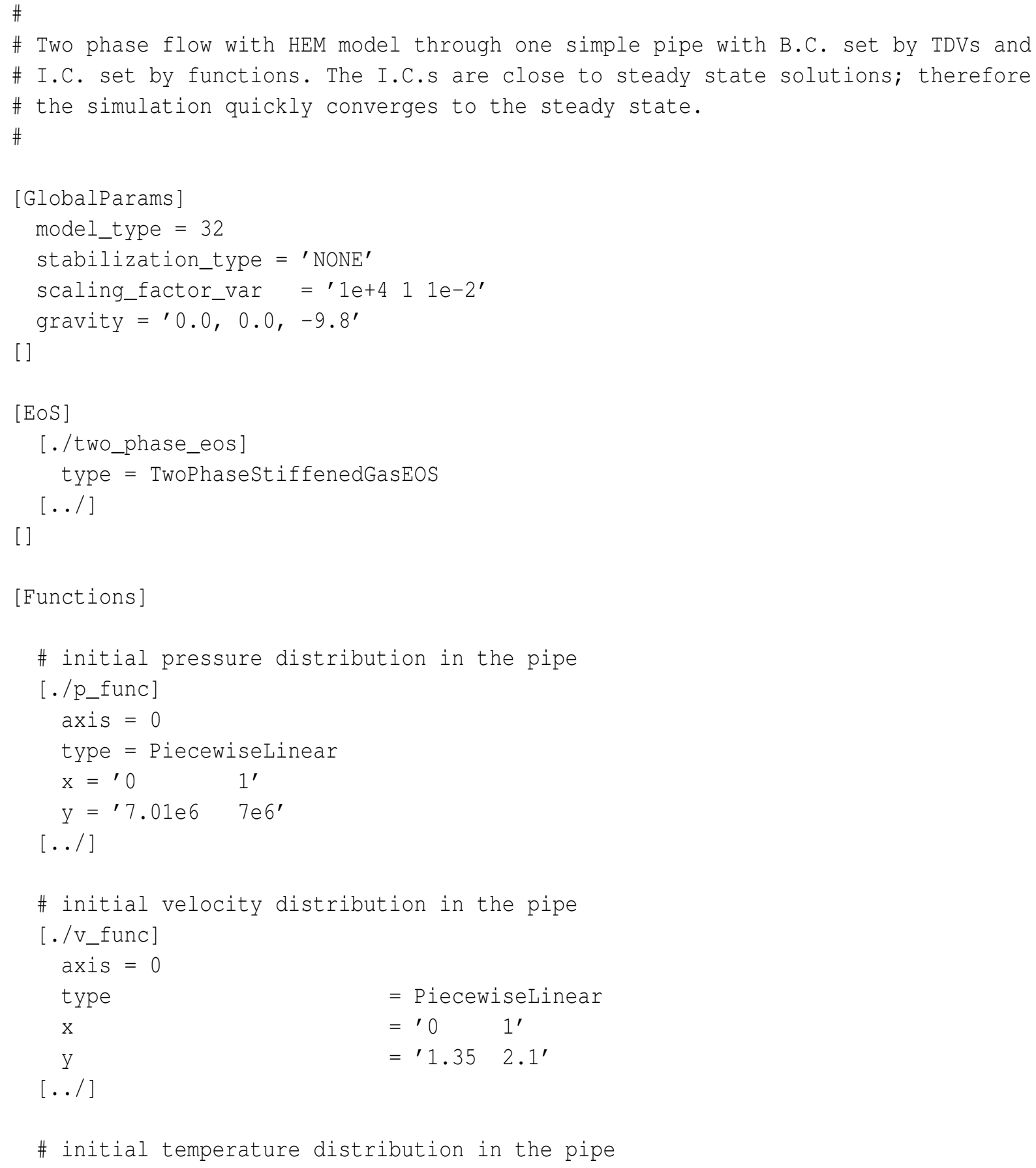




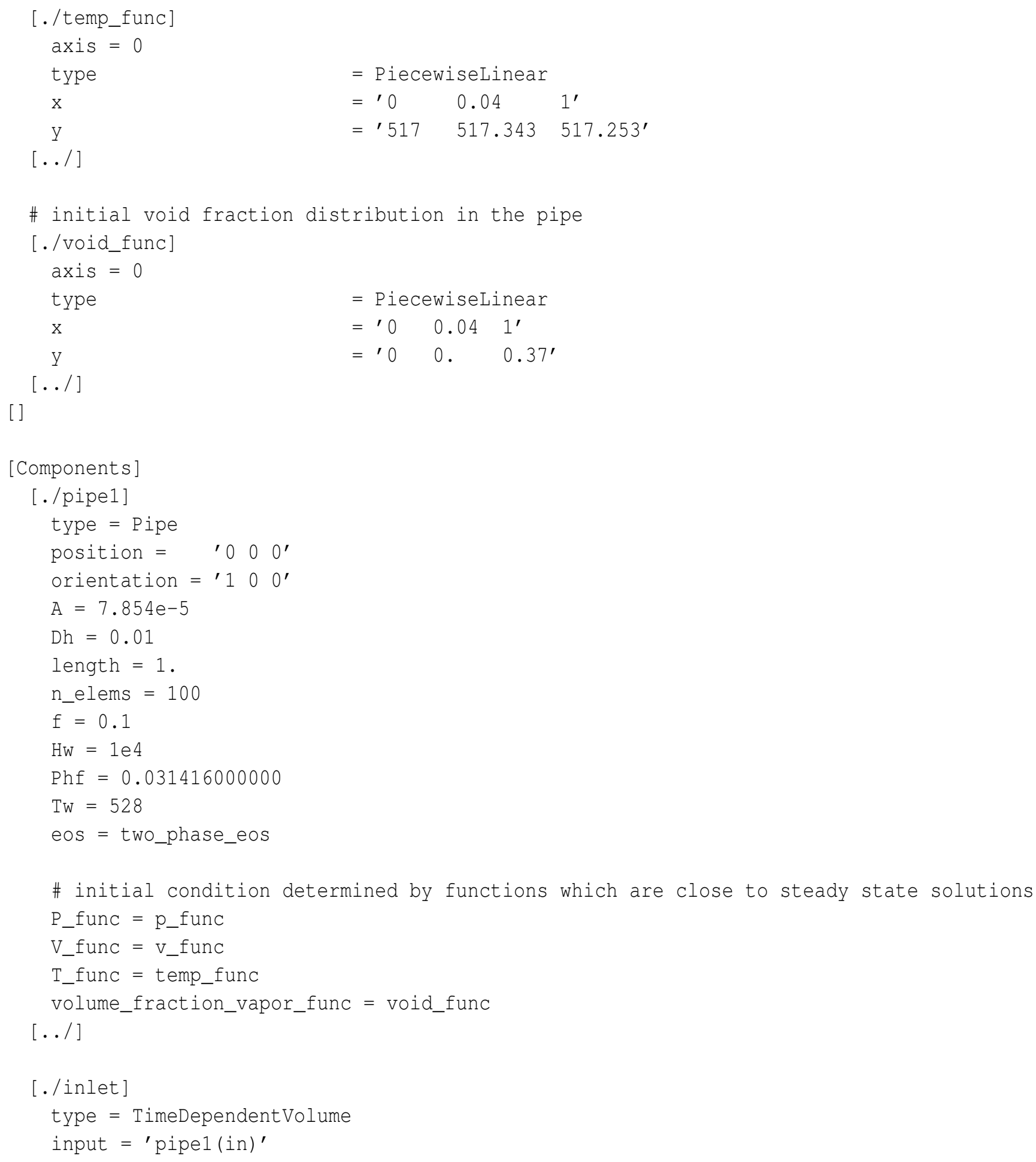




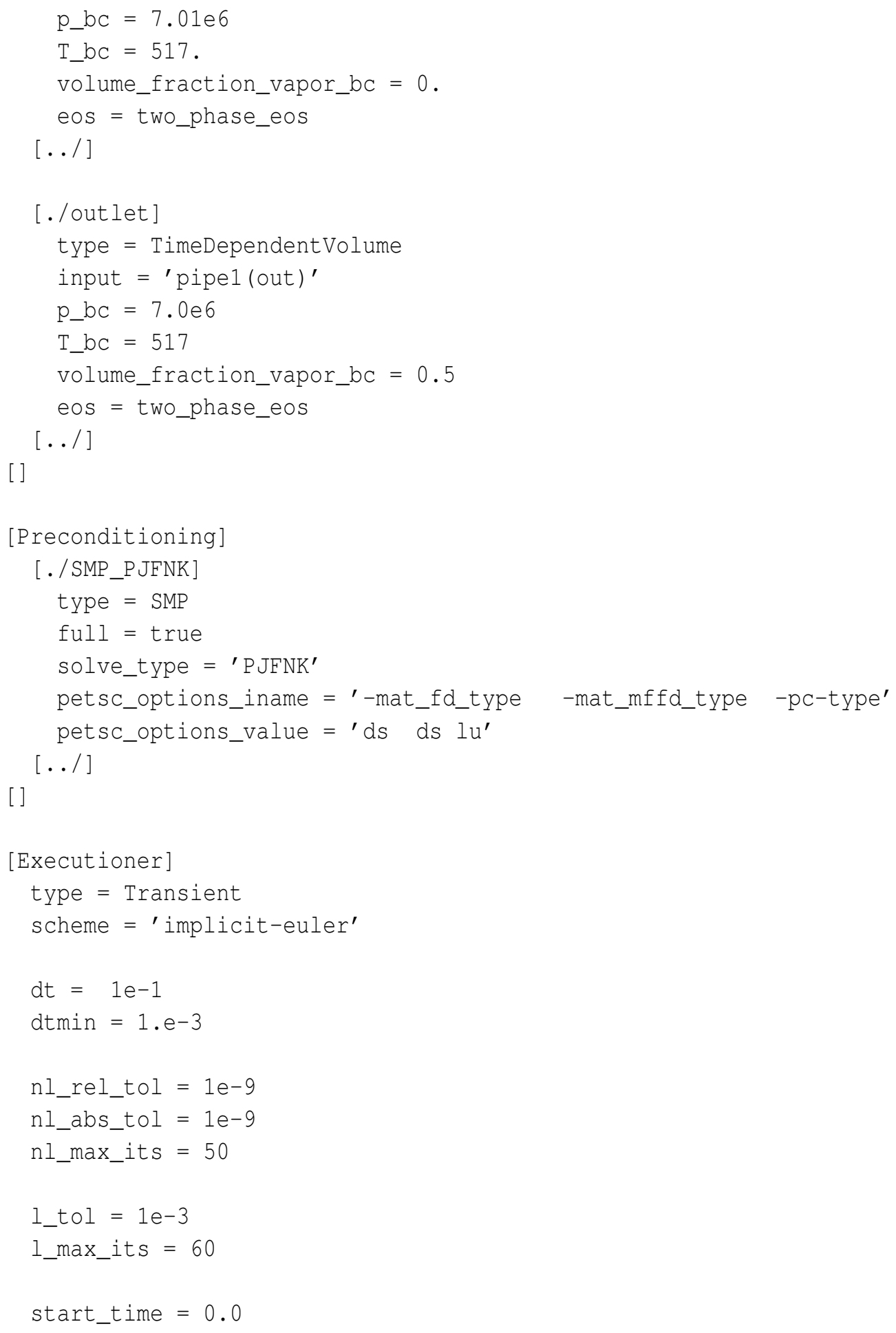




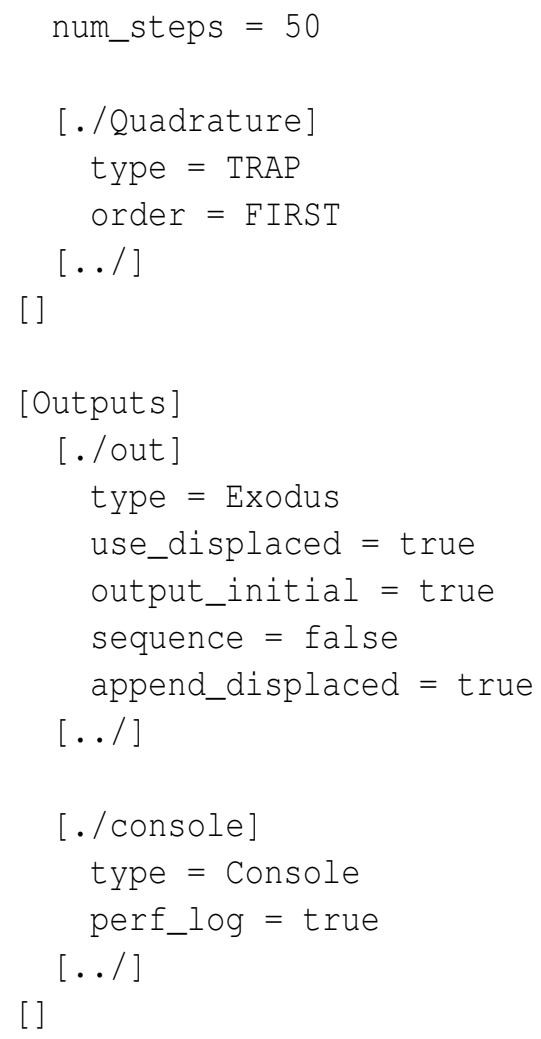

\subsubsection{Description of the Input File}

The following are the detailed explanations of the input parameters that did not exit in the previous examples.

\section{The Global Parameters Block:}

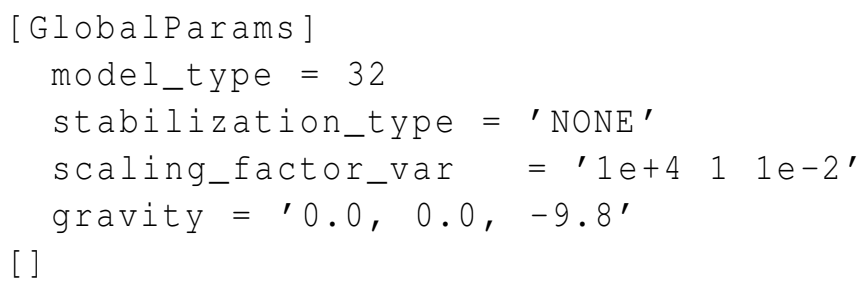

model_type model_type=32 indicates that the HEM two-phase flow model will be used for this problem. 
gravity The gravity acceleration vector.

\section{The Equation of State Block:}

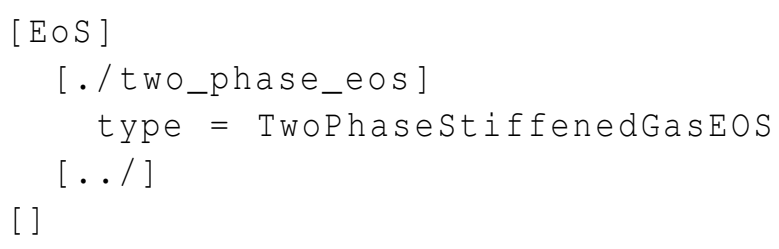

type Two phase stiffened gas equation of state will be used for this problem.

\section{The Components Block:}

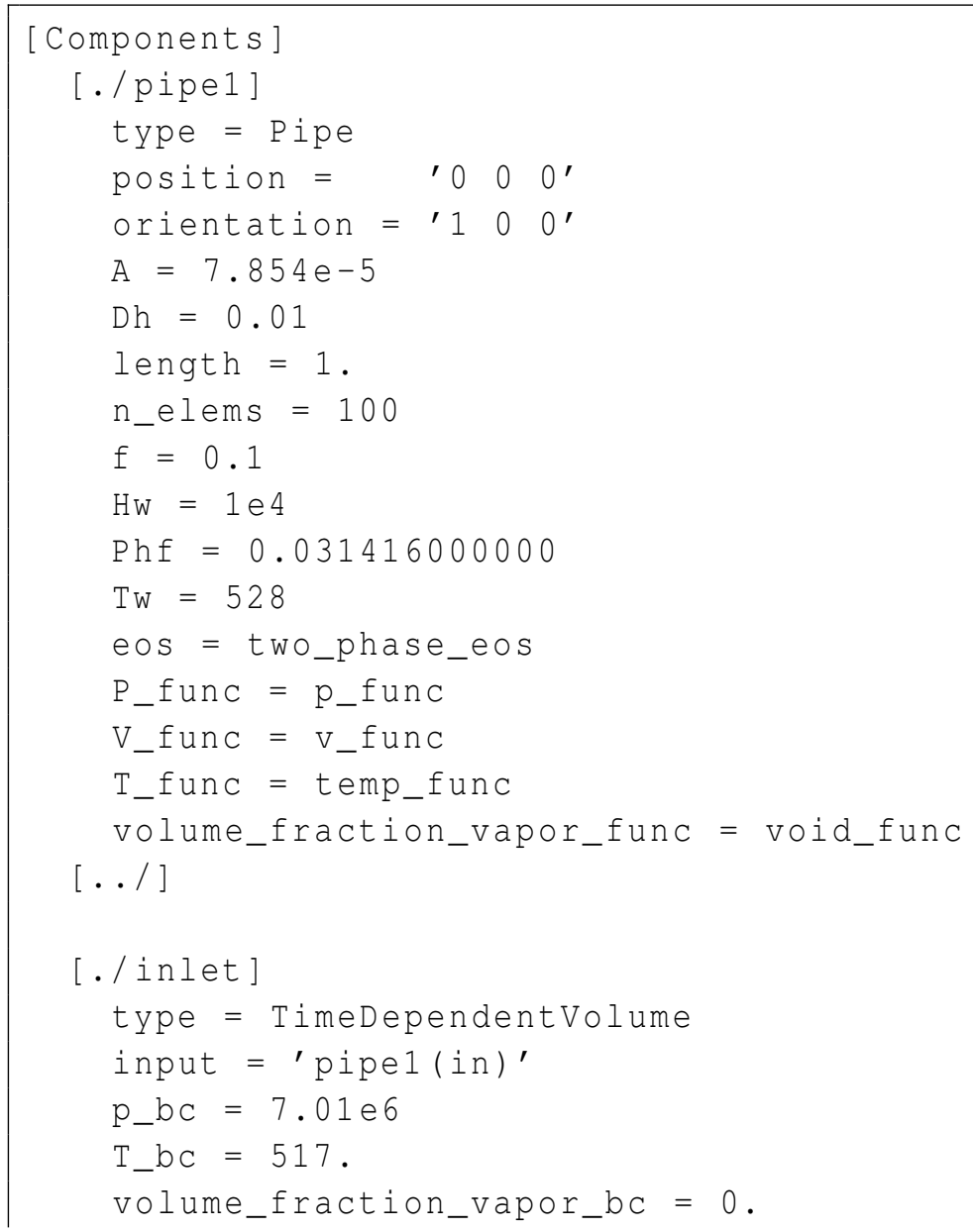




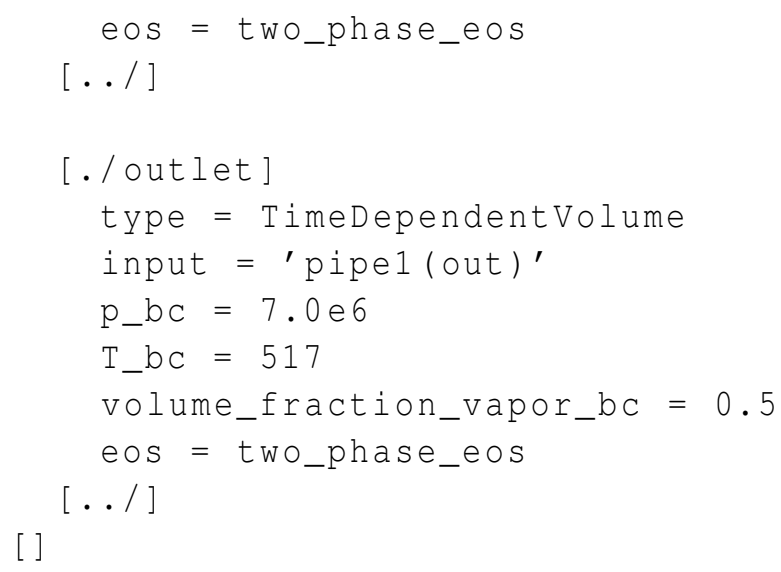

$\mathrm{HW}$

Phf

Tw

$\mathrm{T} \_\mathrm{bc}$

volume_fraction_vapor_bc
The wall convective heat transfer coefficient.

Heat flux perimeter.

Initial pipe wall temperature.

Temperature values at the pipe boundaries.

Vapor phase volume fraction at the pipe boundaries. 


\subsection{Example 4: Two Phase Flow Through a Pipe - 7-Equation Model}

\subsubsection{Problem Description}

This example shows a two phase flow with the 7-equation model through one simple pipe with boundary conditions set by Inlet and Outlet components at the pipe inlet and outlet ends respectively. The initial conditions are set by functions. The initial conditions are set to be close to steady state solutions; therefore the simulation quickly converges to steady state solutions.

\subsubsection{Input File}

The input file for this pipe flow case with the 7-equation two-phase flow model is shown below:

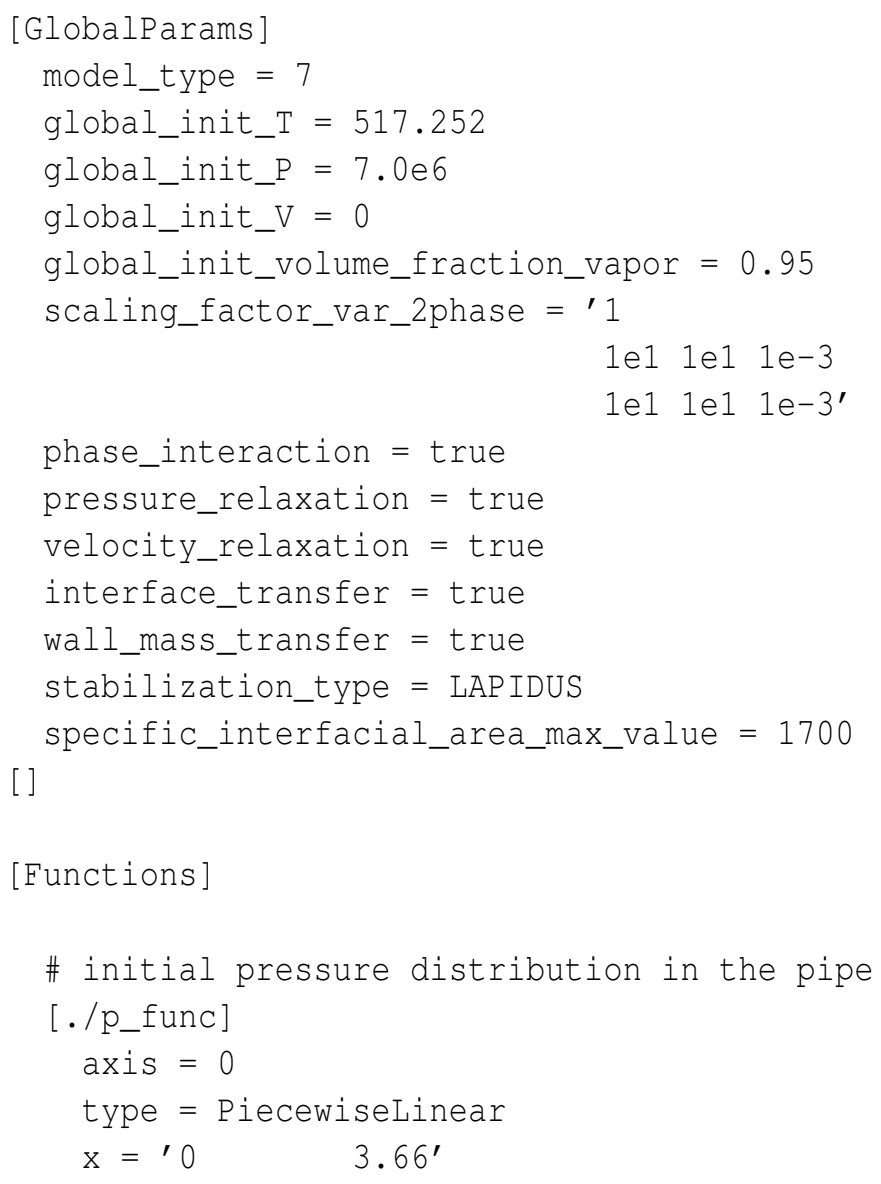




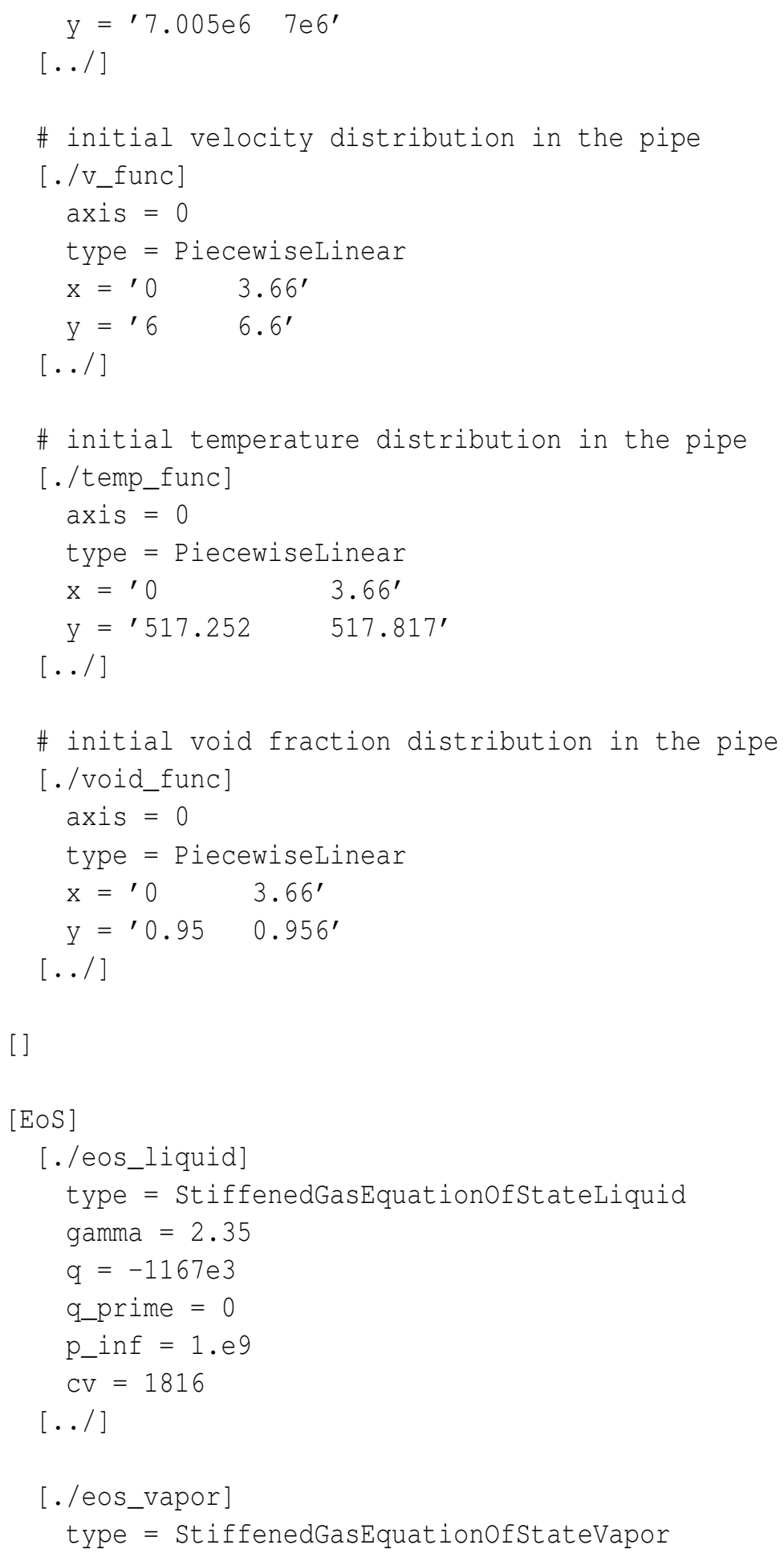




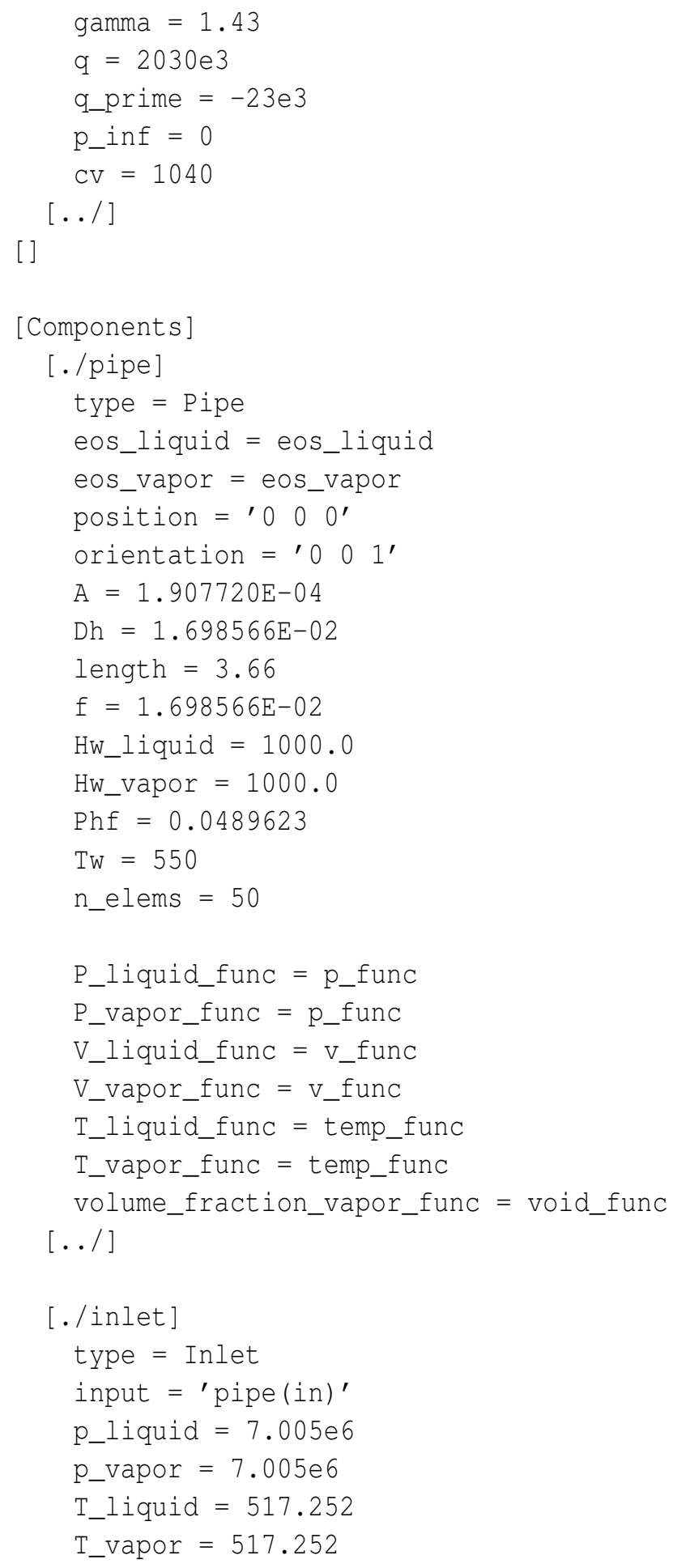




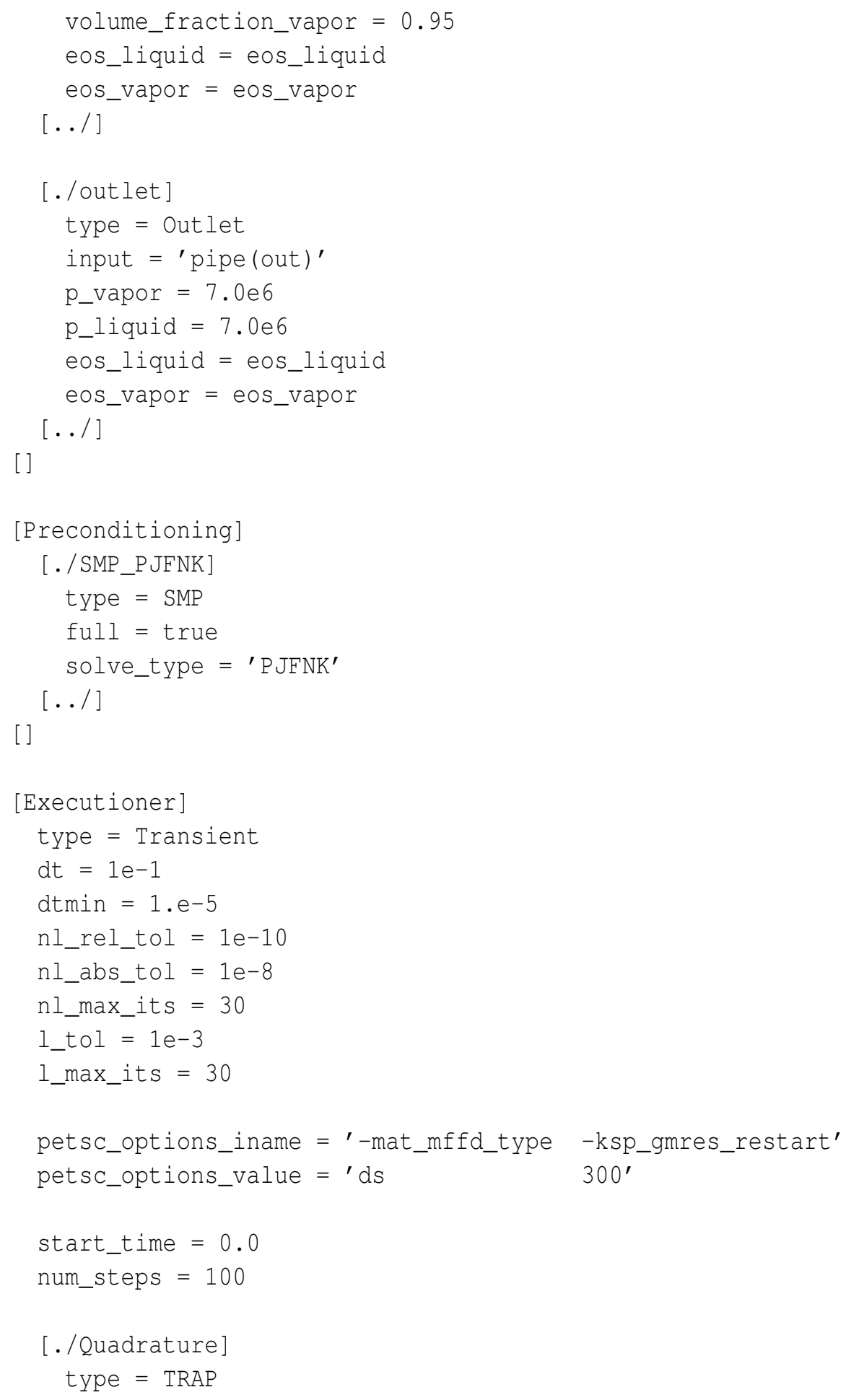




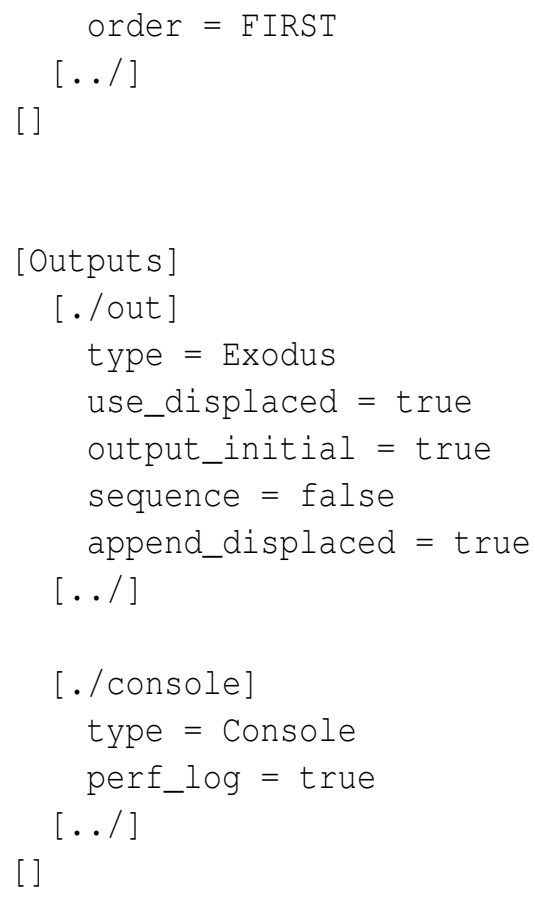

\subsubsection{Description of the Input File}

The following provides the explanations of the input parameters that did not exist in the previous examples:

\section{The Global Parameters Block:}

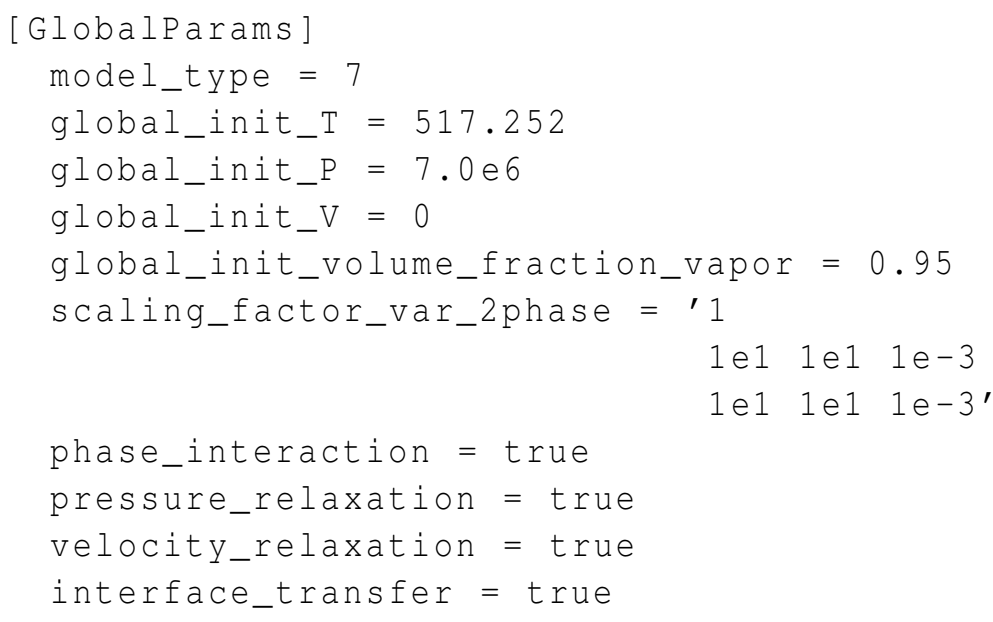




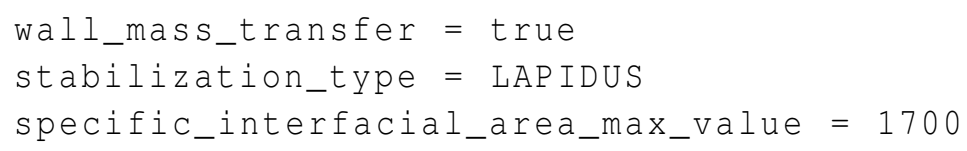

model_type $=7$

scaling_factor_var_2phase

phase_interaction

pressure_relaxation

velocity_relaxation

interface_transfer

wall_mass_transfer

specific_interfacial_area_max_value
Turn on the 7-equation two phase flow model.

Scaling factors for each variable of the 7-equation two phase flow model.

Option to turn on/off the interaction between the two phases.

Option to turn on/off the pressure relaxation terms.

Option to turn on/off the velocity relaxation terms.

Option to turn on/off the interface mass and heat transfer.

Option to turn on/off wall boiling model to calculate the interfacial mass transfer near the wall.

The maximum value of the specific interfacial area.

The Equation of State Block:

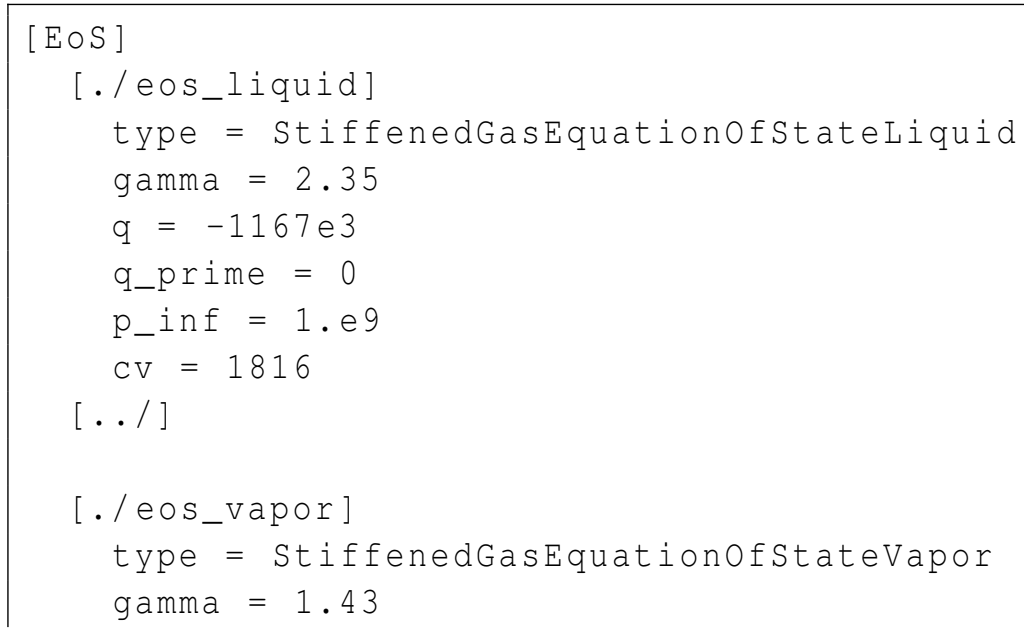




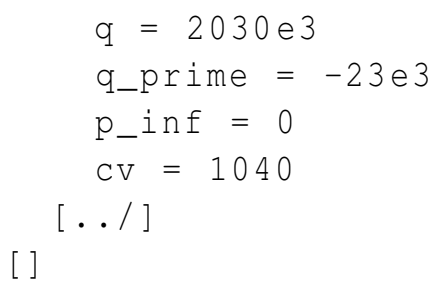

[./eos_liquid] Subblock for the liquid phase equation of state.

type $\quad$ Stiffened gas equation of state (SGEOS) for liquid.

gamma The $\gamma$ value in SGEOS calculations for liquid.

q

The $q$ value in SGEOS calculations for liquid.

q_prime

The $q^{\prime}$ value in SGEOS calculations for liquid.

p_inf

The $p_{\infty}$ value in SGEOS calculations for liquid.

$\mathrm{CV}$

The specific heat value in SGEOS calculations for liquid.

\section{The Components Block}

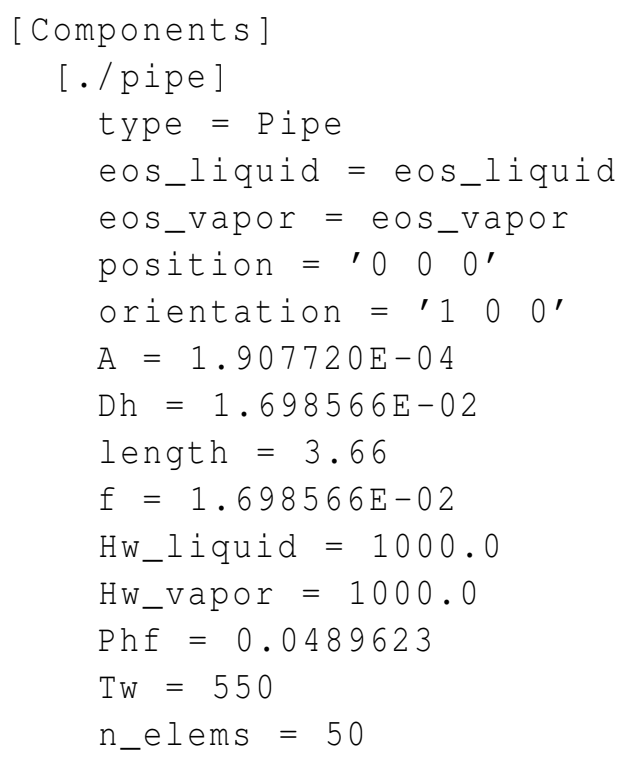




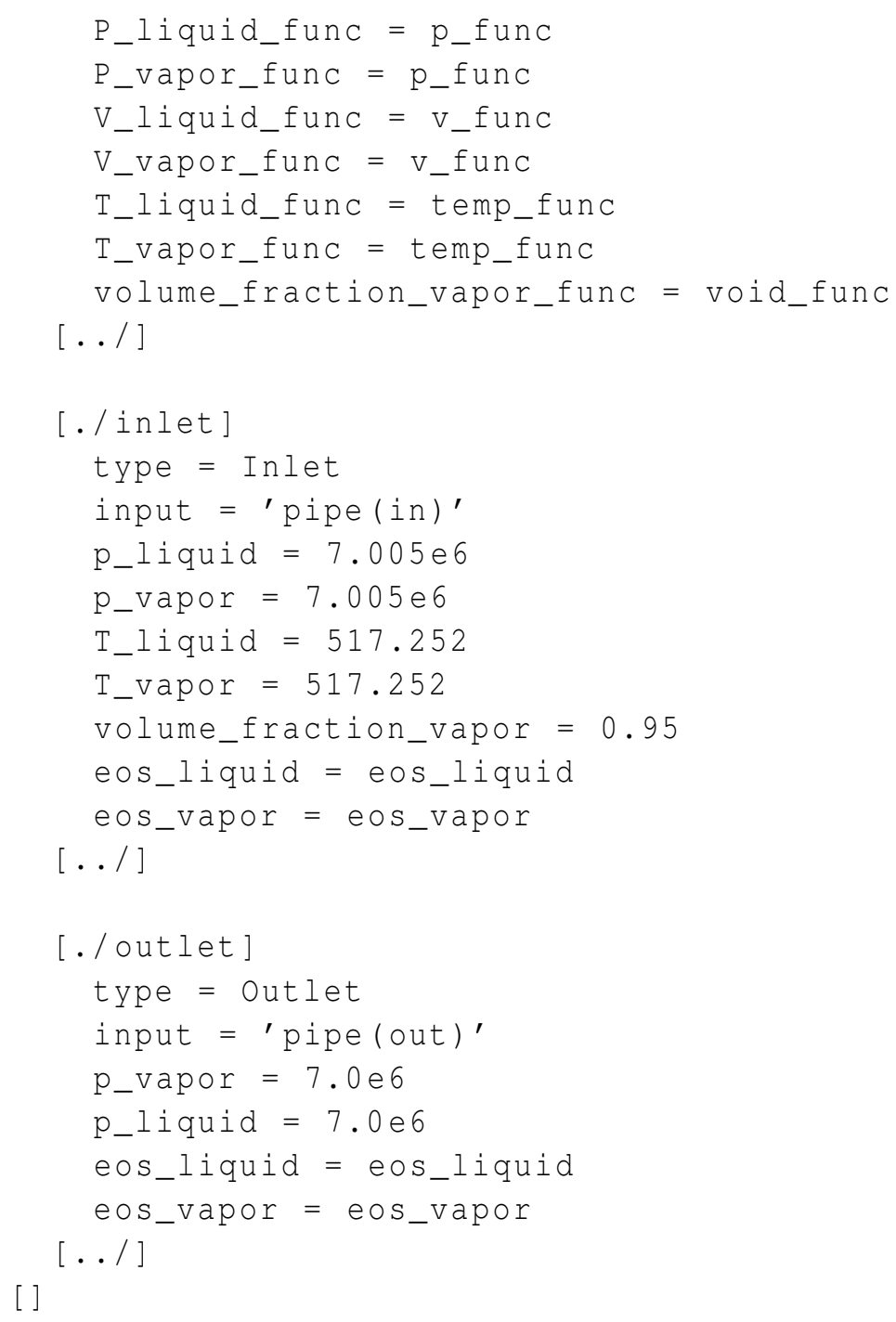

Defines the name of the equation of state to use for the liquid phase.

eos_vapor

Defines the name of the equation of state to use for the vapor phase.

Hw_liquid

Convective heat transfer coefficient for the liquid phase.

Hw_vapor

Convective heat transfer coefficient for the vapor phase. 
P_liquid_func

P_vapor_func

T_liquid_func

T_vapor_func

volume_fraction_vapor_func

p_liquid

p_vapor

T_liquid

T_vapor

volume_fraction_vapor
Function which defines liquid phase pressure as a function of $\mathrm{x}$.

Function which defines vapor phase pressure as a function of $\mathrm{x}$.

Function which defines liquid phase temperature as a function of $x$.

Function which defines vapor phase temperature as a function of $x$.

Function which defines vapor phase volume fraction as a function of $x$.

Prescribed pressure value at the pipe boundary for the liquid phase.

Prescribed pressure value at the pipe boundary for the vapor phase.

Prescribed temperature value at the pipe boundary for the liquid phase.

Prescribed temperatur value at the pipe boundary for the vapor phase.

Prescribed vapor phase volume fraction. 


\subsection{Example 5: A Core Channel Problem}

\subsubsection{Problem Description}

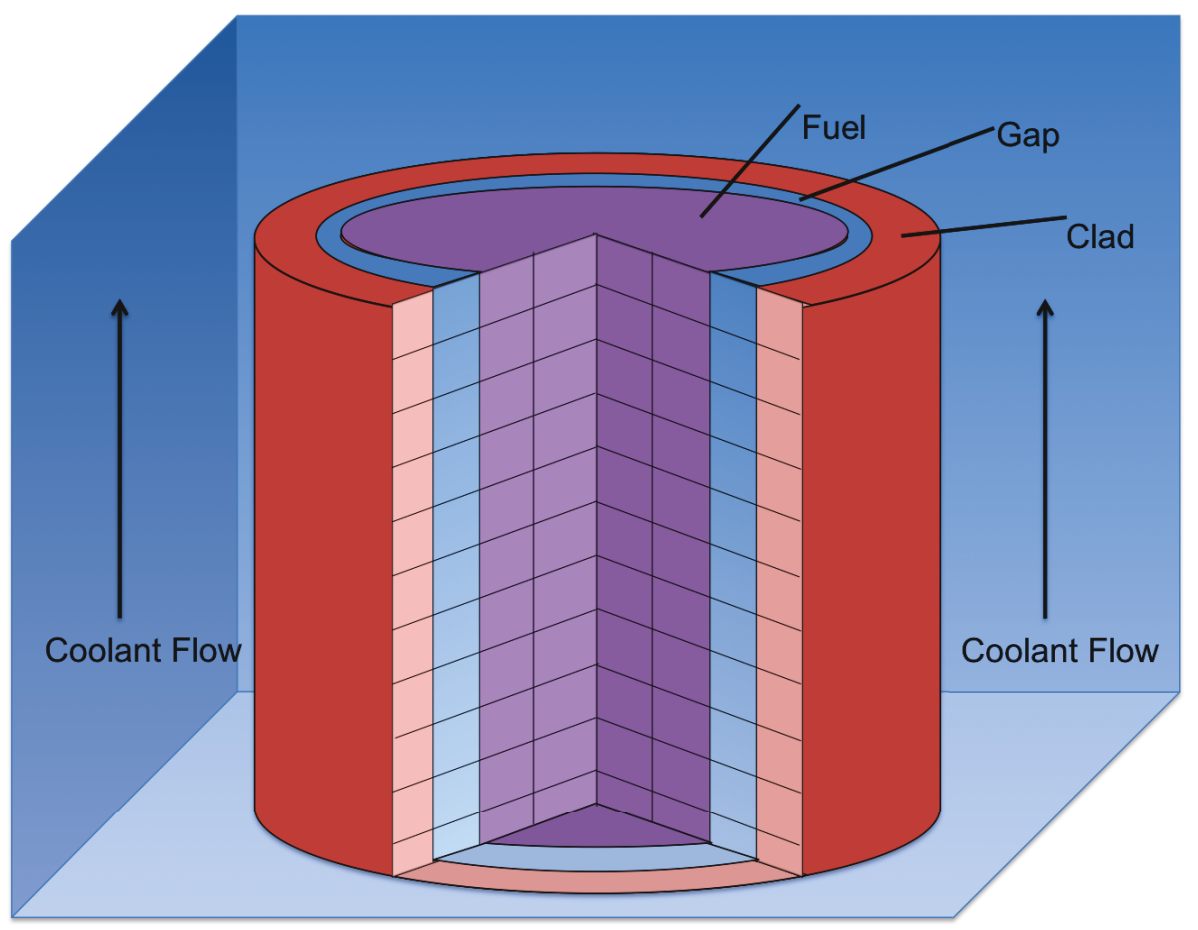

Figure 4. Diagram of a core channel problem

This example simulates single phase fluids flow and heat transfer in a core channel, as schematically shown in Fig. 4. This problem similates fluids flow in one subchannel with a single fuel rod as the heat source. The boundary conditions are applied to the ends of the core channel. The reactor power $=77337.69407 \mathrm{~W}$ is used for the core channel. Other parameters used are the following:

Fluids Flow:

Cross section flow area $=8.7878 \mathrm{e}-5 \mathrm{~m}^{2}$

Hydraulic Diameter $=0.01179 \mathrm{~m}$

Length $=4 \mathrm{~m}$

Friction coefficient $=0.01$

Convective heat transfer coefficient $=5.33 \mathrm{e} 4 \mathrm{~W} / \mathrm{m}^{2} * K$ 
Heat flux perimeter $=0.029832559676 \mathrm{~m}$

Number of elements $=20$

Heat Structure:

Dimension of mesh used for heat conduction $=1$

Geometry type of fuel $=$ cylinder

Heat structure names = 'fuel gap clad'

Number of heat structures $=3$

Width of each heat structure $=$ '0.0040960.00010.000552' $\mathrm{m}$

Number of elements of each heat structure $=$ '10 12 ',

Name of materials used in the heat structure = 'fuel-mat gap-mat clad-mat'

Fraction of reactor power goes into heat structure $=$ ' 1.00 .00 .0 '

Initial Solid Temperature $=559.15 \mathrm{~K}$

The boundary conditions are the following:

Inlet:

Pressure $=155.483 \mathrm{e} 5 \mathrm{~Pa}$

Temperature $=559.15 \mathrm{~K}$

Outlet:

Pressure $=155 . \mathrm{e} 5 \mathrm{~Pa}$

Temperature $=559.15 \mathrm{~K}$

The solid material properties $(\mathrm{k}=$ Thermal conductivity, $\mathrm{Cp}=$ heat capacity, rho $=$ Density $)$ are:

Fuel:

$\mathrm{k}=2.5$

$\mathrm{Cp}=300$

rho $=1.032 \mathrm{e} 4$

Gap:

$\mathrm{k}=0.6$

$\mathrm{Cp}=1$.

rho $=1$.

Clad:

$\mathrm{k}=21.5$

$\mathrm{Cp}=350$. 
rho $=6.55 \mathrm{e} 3$

\subsubsection{Input File}

The follwoing shows the input file for the Core Channel example. Please note that the mesh used for this problem does not correspond to what is shown in Fig. 4.

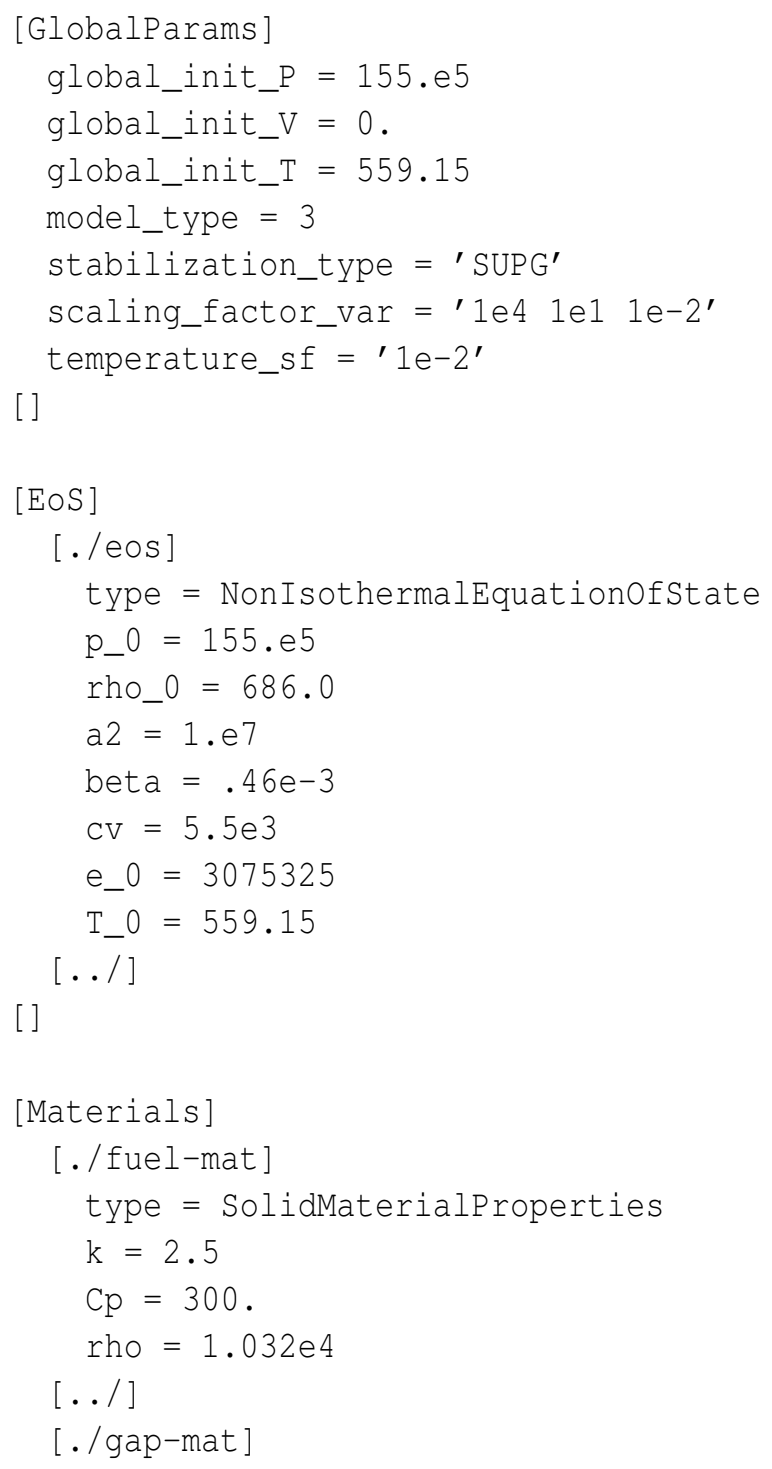




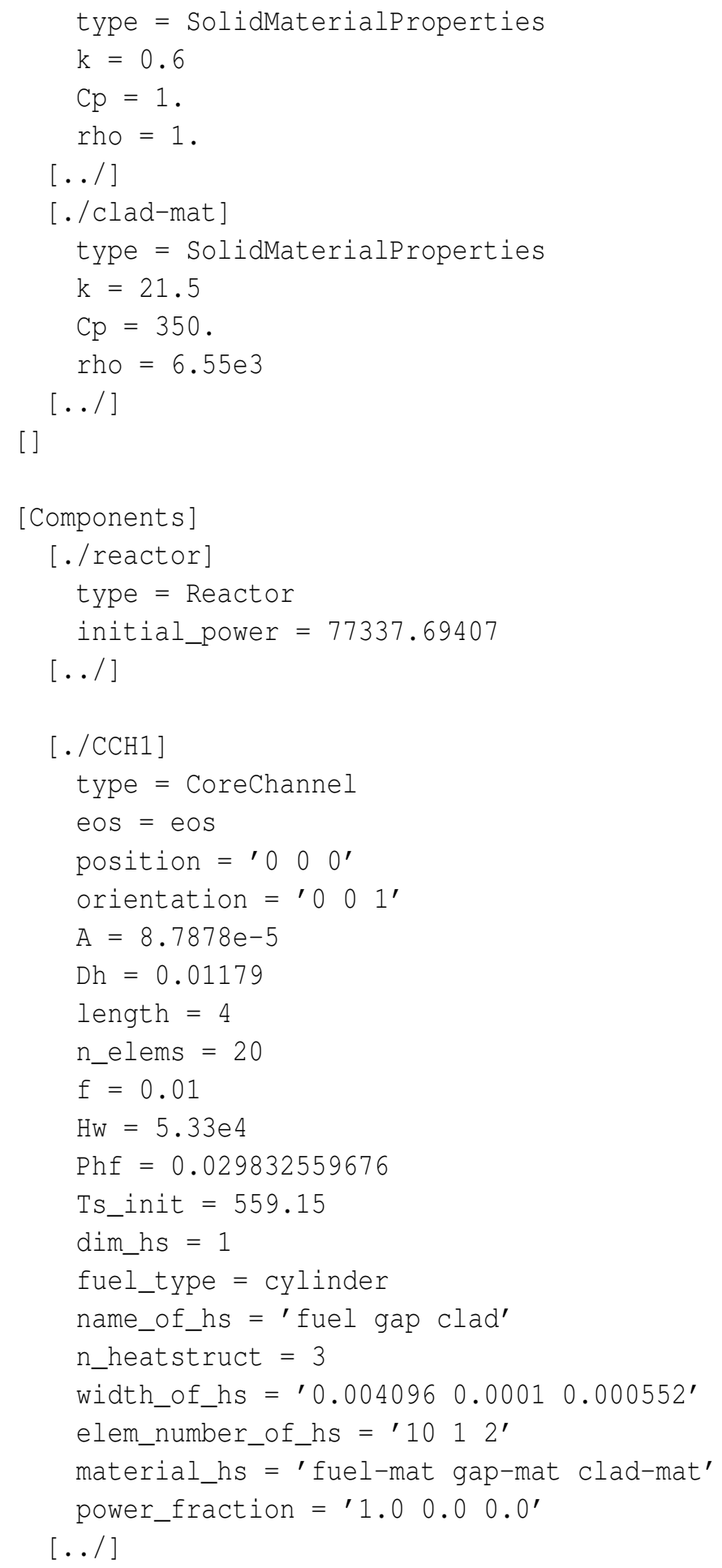




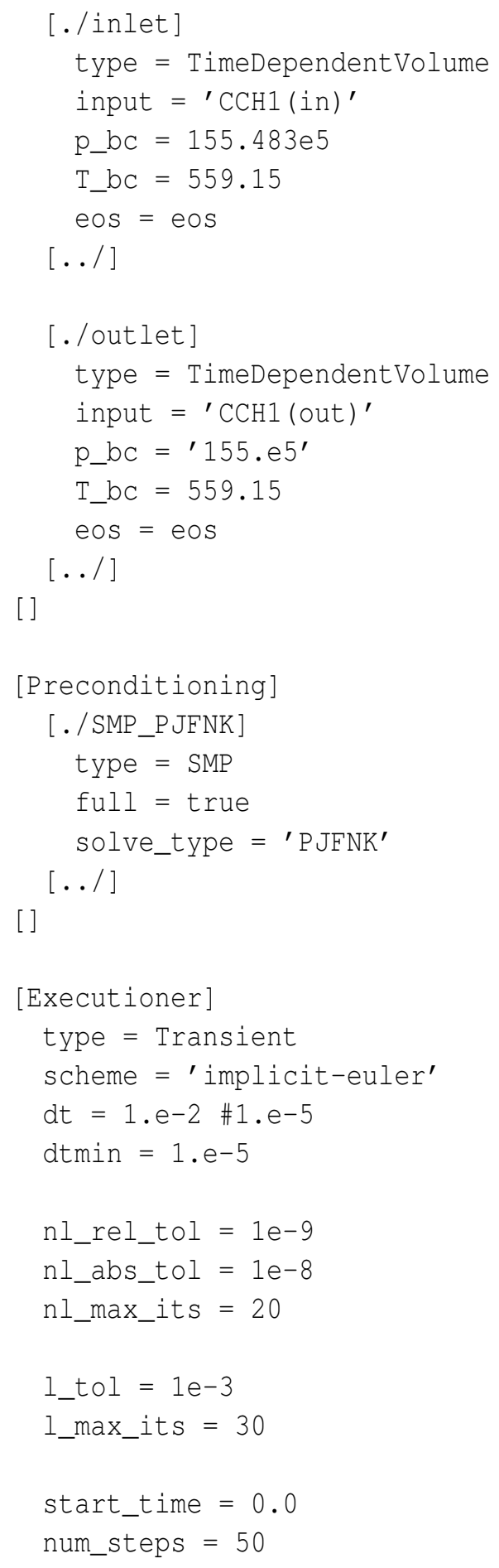




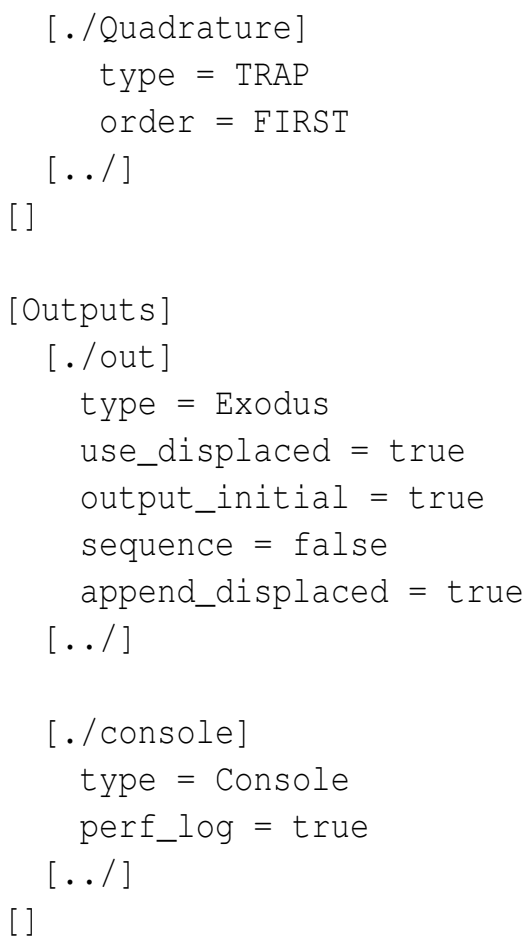

\subsubsection{Description of the Input File}

\section{The Global Parameter Block:}

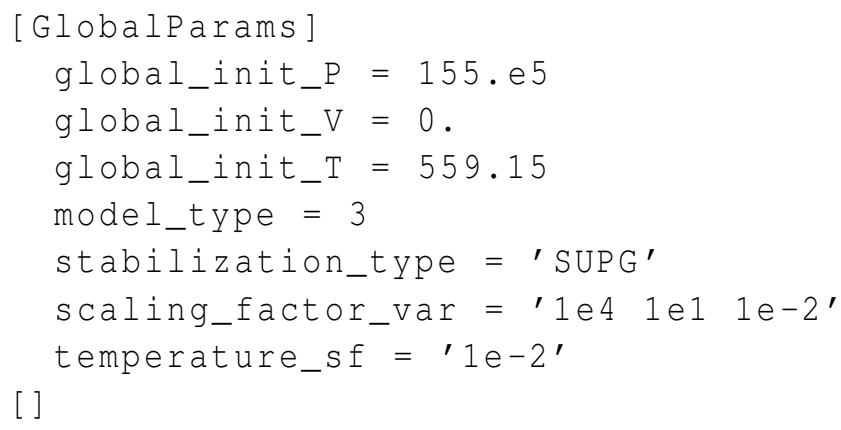

temperature_sf Scaling factor for the temperature variable in solid materials.

\section{The Materials Block:}




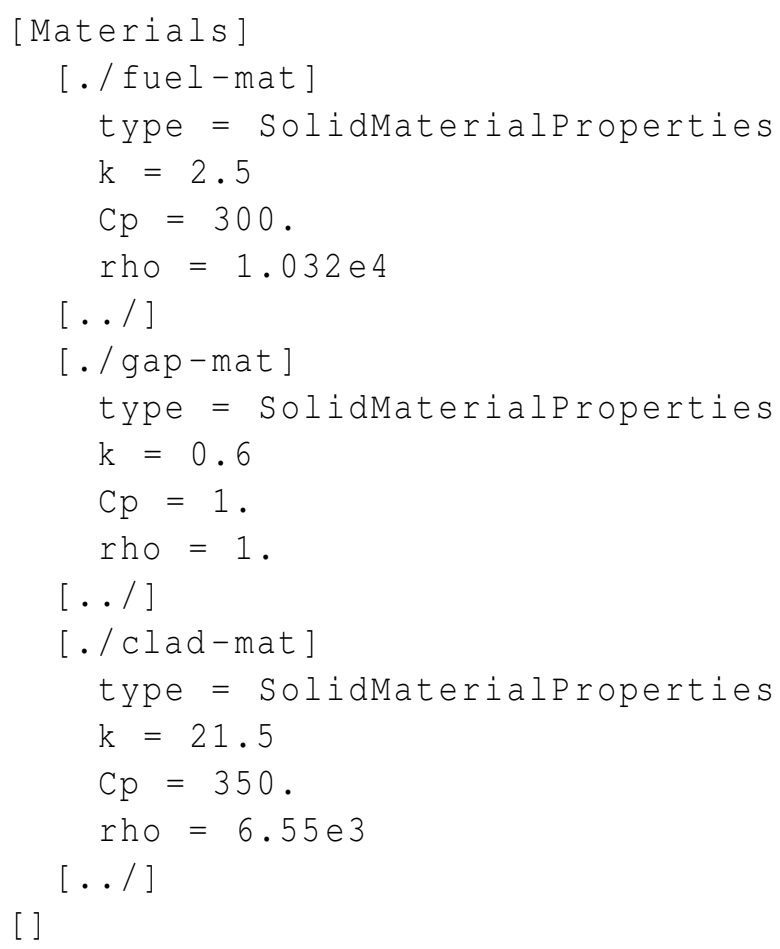

k Thermal conductivity.

$\mathrm{Cp} \quad$ Specific heat.

rho Density of solid materials.

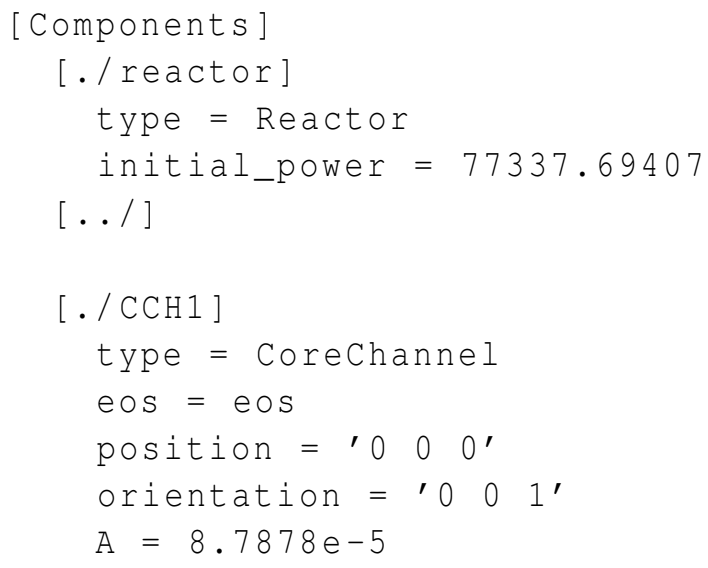




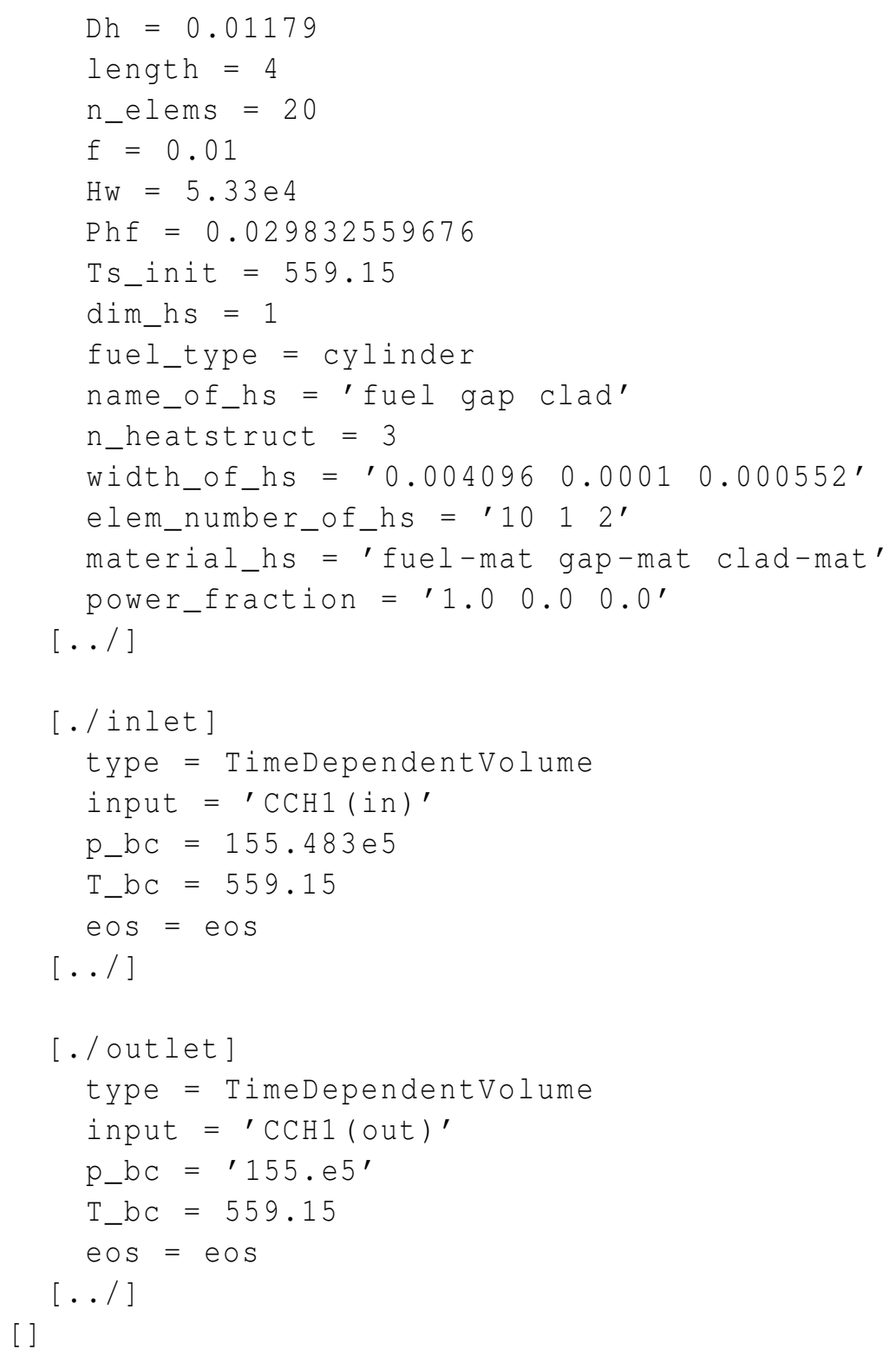


$\mathrm{HW}$

Ts_init

dim_hs

fuel_type

name_of_hs

n_heatstruct

width_of_hs

elem_number_of_hs

material_hs

power_fraction
Wall heat transfer coefficient.

Prescribed initial temperature of the solid materials.

The dimension of the mesh used for the heat conduction calculations in the heat structure. The options are dim_hs=1 for 1D heat conduction calculations or dim_hs $=2$ for $2 \mathrm{D}$ heat conduction calculations.

Geometry type of the fuel. The available options are fuel_type = cylinder or fuel_type = plate.

Prescribed heat structure names.

Prescribed number of heat structures.

Width of each heat structure.

Number of elements of each heat structure.

Name of the materials (defined in the Materials block) used in the heat structure.

The fraction of reactor power that goes into each heat structure. 


\subsection{Example 6: A Two Pipes Flow Problem}

\subsubsection{Problem Description}

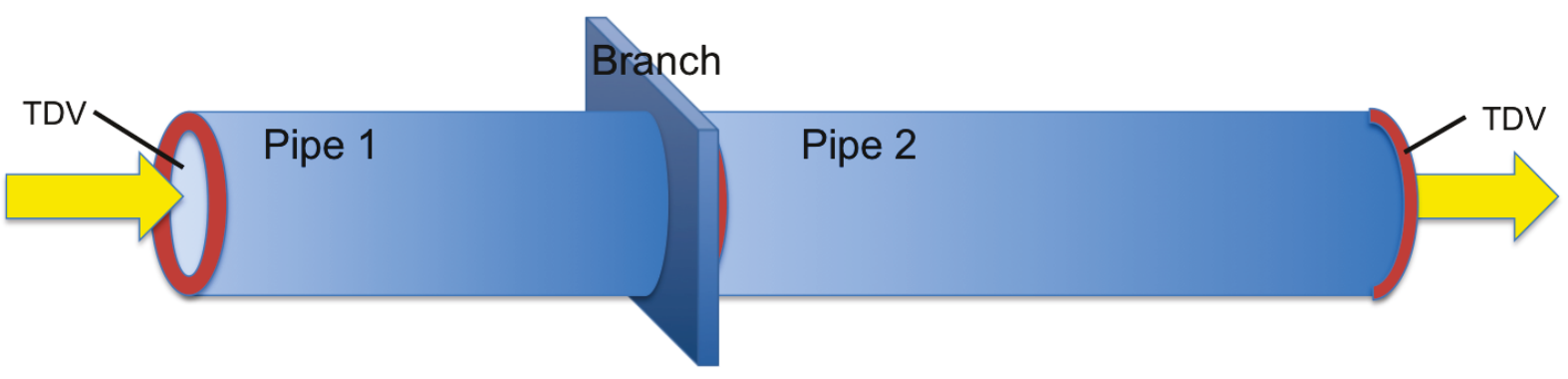

Figure 5. Diagram of a two pipes flow problem

This example demonstrates flow through two pipes with different parameters connected by a branch. There is no heat exchange through the pipe walls for this case. The following are the input parameters for each component:

Pipe 1:

Hydraulic Diameter $=.01 \mathrm{~m}$

Area $=1.0 \mathrm{e}-4 \mathrm{~m}^{2}$

Length $=1 \mathrm{~m}$

Friction coefficient $=.01$

Convective heat transfer coefficient $=0$

Pipe 2:

Hydraulic Diameter $=.02 \mathrm{~m}$

Area $=4 \mathrm{e}-4 \mathrm{~m}^{2}$

Length $=1.5 \mathrm{~m}$

Friction $=.02$

Convective Heat Transfer Coefficient $=0$

Branch:

Form loss coefficients $=$ ' 5 5'

Area $=1.5 \mathrm{e}-4 m^{2}$ 
Initial pressure $=1 \mathrm{e} 5 \mathrm{~Pa}$

Inlet:

Pressure $=1.01 \mathrm{e} 5 \mathrm{~Pa}$

Temperature $=300 \mathrm{~K}$

Outlet:

Pressure $=1 \mathrm{e} 5 \mathrm{~Pa}$

The following figure shows the results of the pressure versus pipes length for this case.

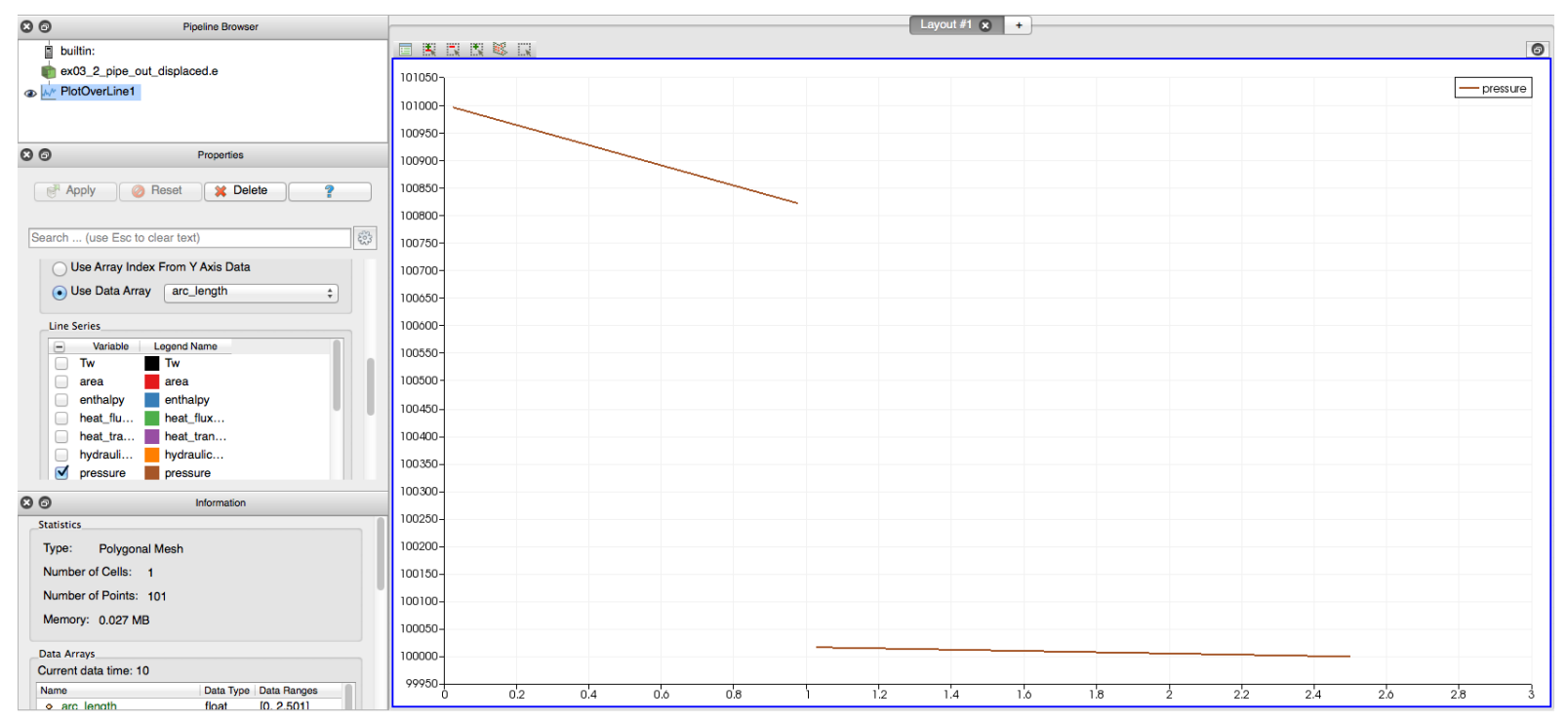

Figure 6. Pressure vs. length for the two pipes flow problem

The results of the pressure difference can be checked by doing hand calculations by summing $\Delta P$ using:

$$
\Delta P=\frac{f L \rho u^{2}}{2 D_{h}}
$$

and:

$$
\Delta P=\frac{k \rho u^{2}}{2}
$$




\subsubsection{Input File}

The input file for this example problem is listed as follows:

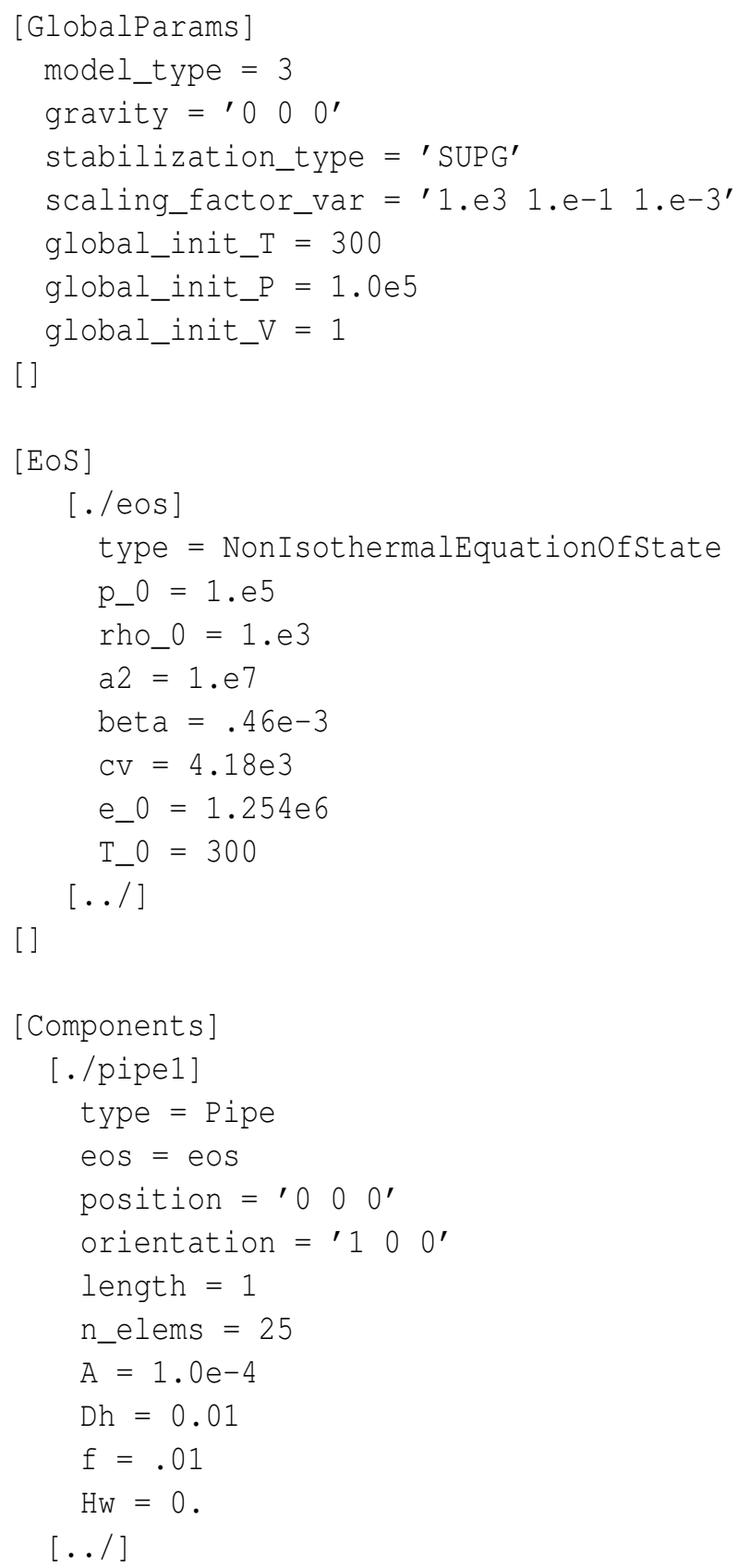




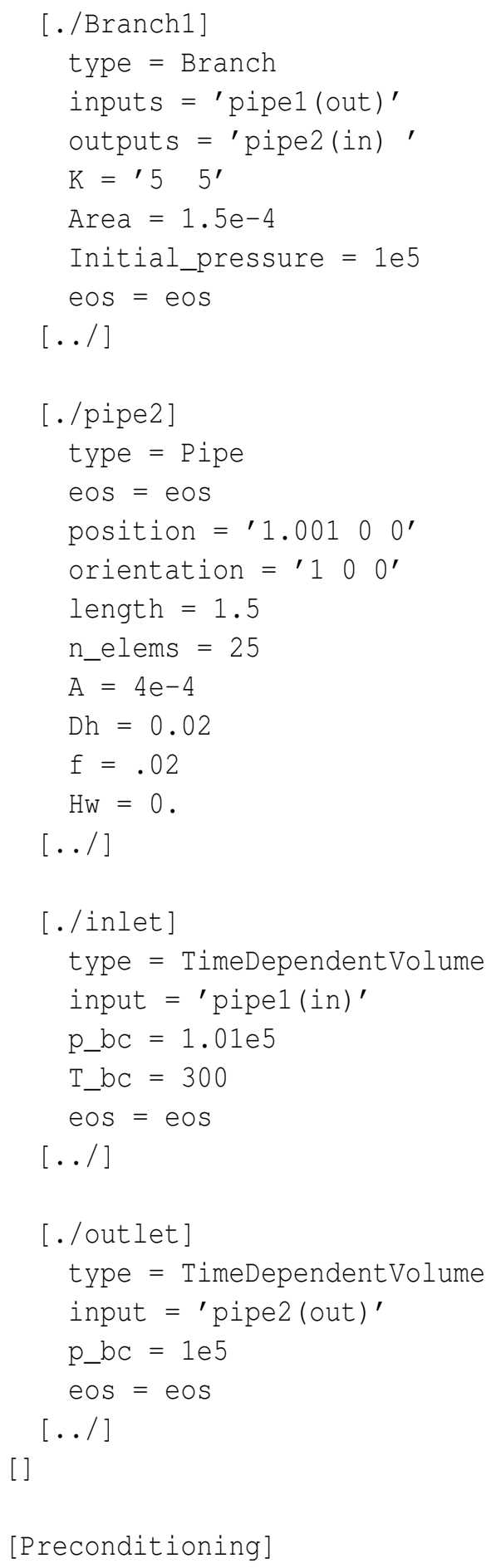




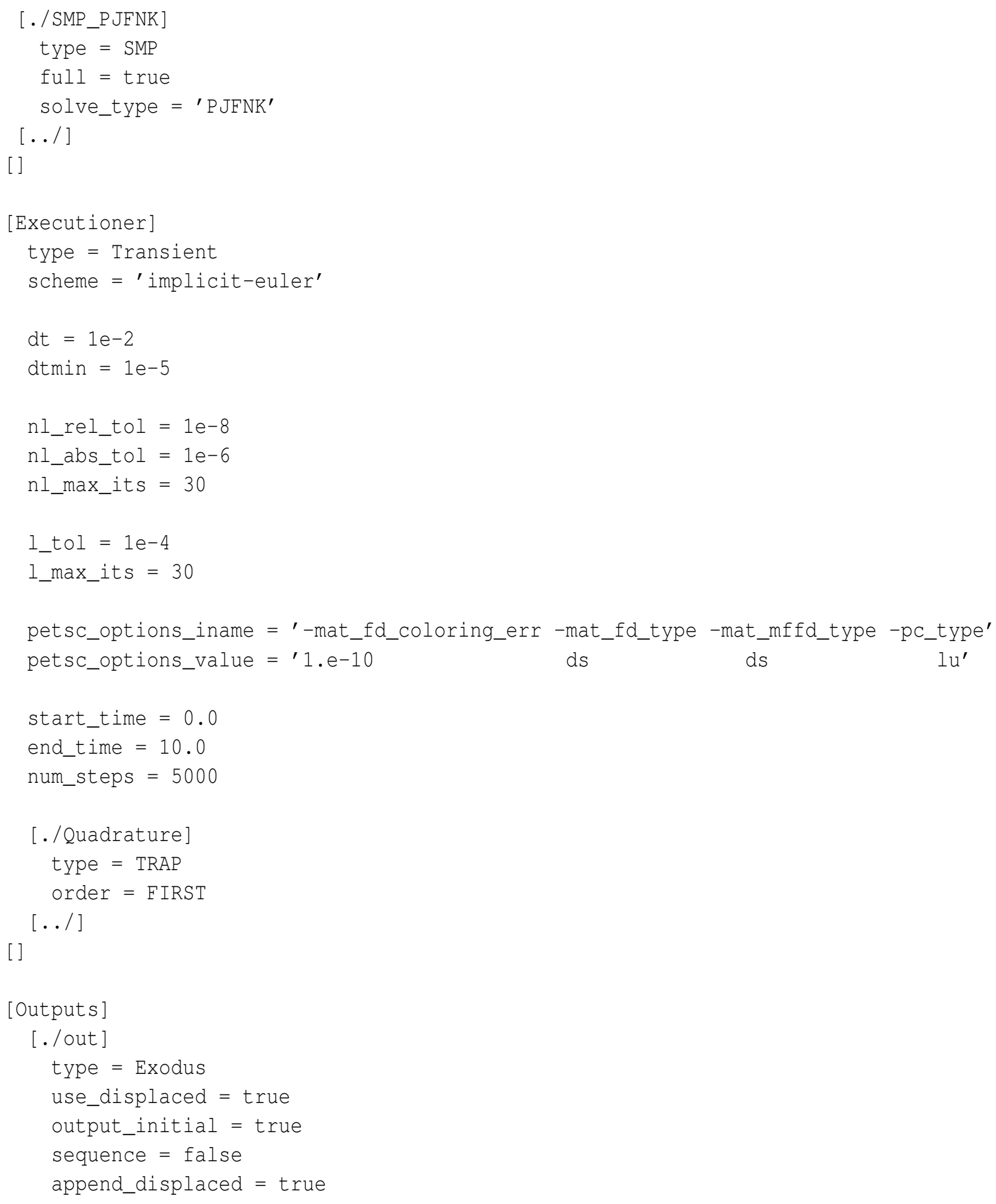




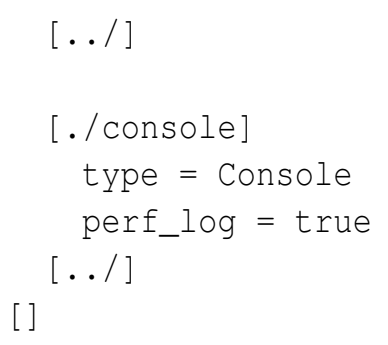

\subsubsection{Description of the Input File}

The only input parameters that need explanations are those in the Branch subblock.

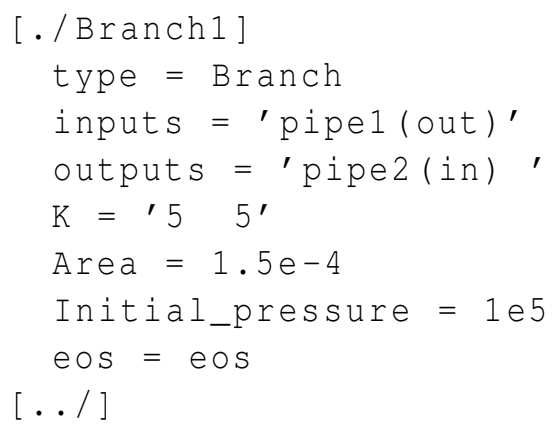

[./Branch $\quad$ Subblock for the Branch component.

type The component type is Branch.

inputs Inputs of this branch connects to the outlet end of pipe1.

outputs Outputs of this branch connects to the inlet end of pipe2.

K Form loss coefficients at the branch inputs and outputs.

Area The reference area of this branch.

Initial_pressure Prescribed initial pressure for the branch.

eos The name of the equation of state to be used for this branch. 


\subsection{Example 7: A Volume Branch Case - Three Pipes In and Two Pipes Out}

\subsubsection{Problem Description}

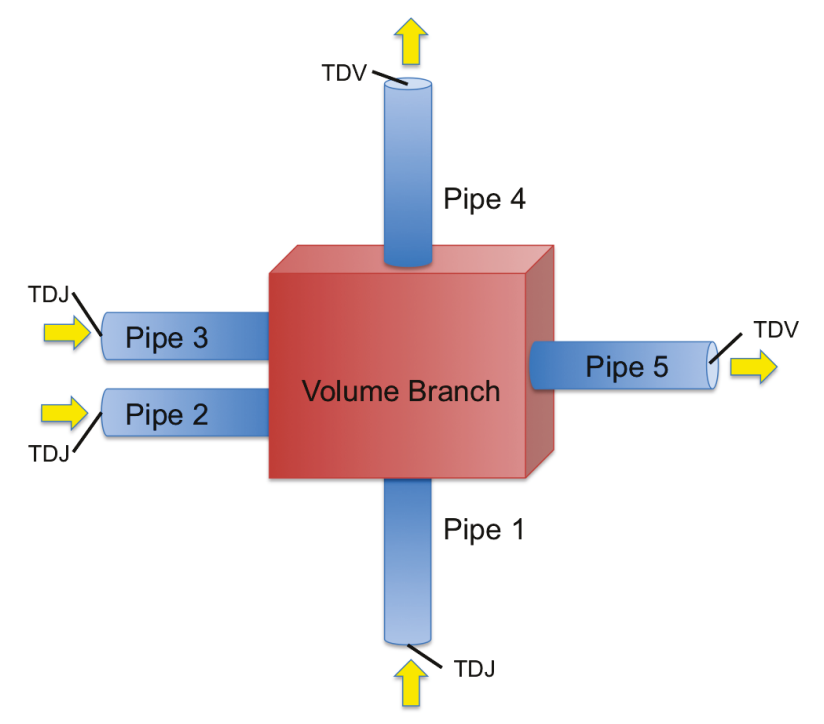

Figure 7. Diagram of a volume branch case with three pipes flowing in and two pipes flowing out

This example shows a volume branch case which connects three pipes with water flowing into them and two pipes with water flowing out of them. The pipe sizes are all the same, but the boundary conditions vary. The volume branch model includes the volume effects of a junction. The input parameters used in this example are listed in the following:

Pipes:

Area $=3.14 \mathrm{e}-4 m^{2}$

Diameter $=0.02 \mathrm{~m}$

Length $=1 \mathrm{~m}$

Number of elements in pipe $=10$

Friction coefficient $=0.01$ 
Branch1:

Type $=$ VolumeBranch

inputs = 'pipe1(out) pipe2(out) pipe3(out)'

outputs = 'pipe4(in) pipe5(in)'

Form loss coefficients $=$ '0.010.010.010.01 100'

Area $=3.14 e-2 m^{2}$

volume $=3.14 e-2 m^{3}$

Initial Temperature $=628.15 \mathrm{~K}$

Inlet 1:

type $=$ TimeDependentJunction

Velocity $=1.0 \mathrm{~m} / \mathrm{s}$

Temperature $=628.15 \mathrm{~K}$

Inlet 2:

type $=$ TimeDependentJunction

Velocity $=1.0 \mathrm{~m} / \mathrm{s}$

Temperature $=628.15 \mathrm{~K}$

Inlet 3:

type $=$ TimeDependentJunction

Velocity $=10.0 \mathrm{~m} / \mathrm{s}$

Temperature $=528.15 \mathrm{~K}$

Outlet 1:

type $=$ TimeDependent Volume

Pressure $=1.0 \mathrm{e} 5 \mathrm{~Pa}$

Temperature $=628.15 \mathrm{~K}$

Outlet 2:

type $=$ TimeDependentVolume

Pressure $=1.5 \mathrm{e} 5 \mathrm{~Pa}$

Temperature $=628.15 \mathrm{~K}$ 


\subsubsection{Input File}

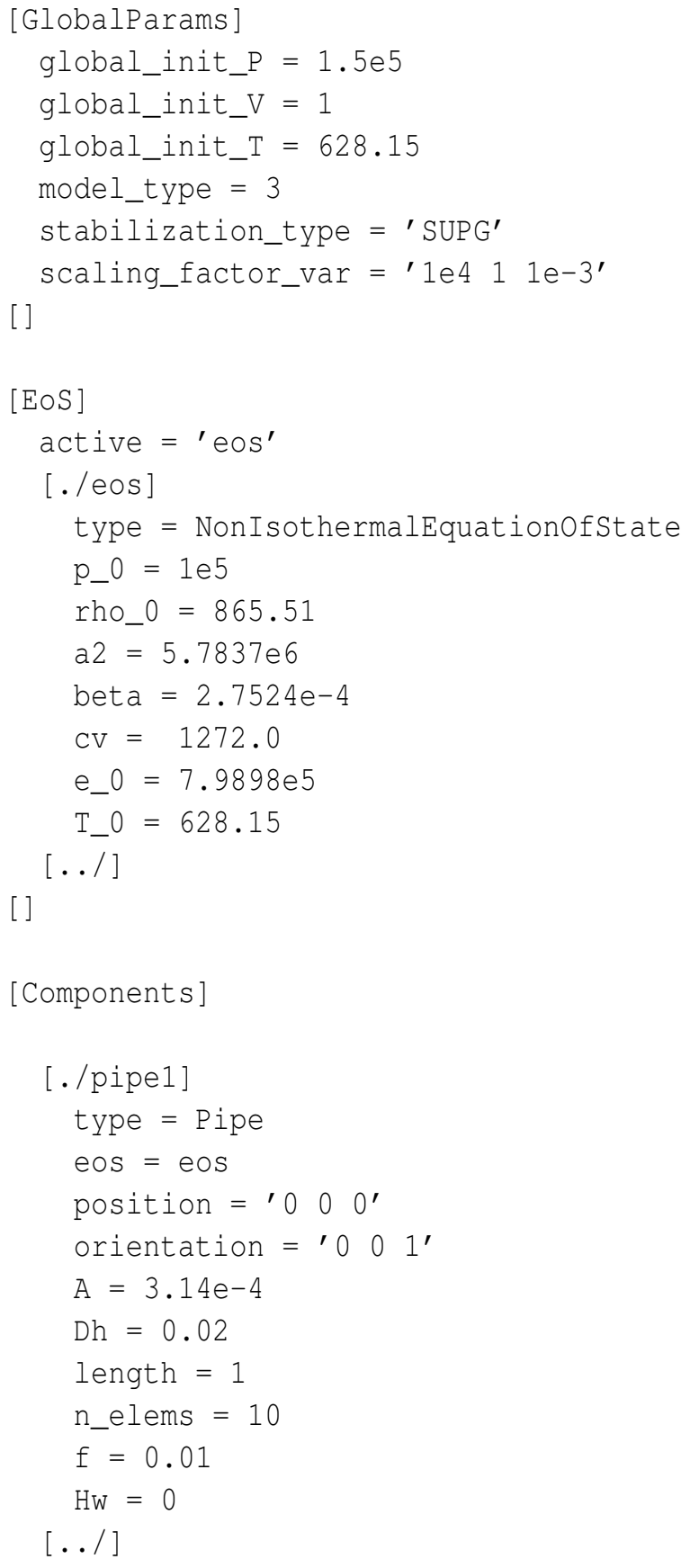




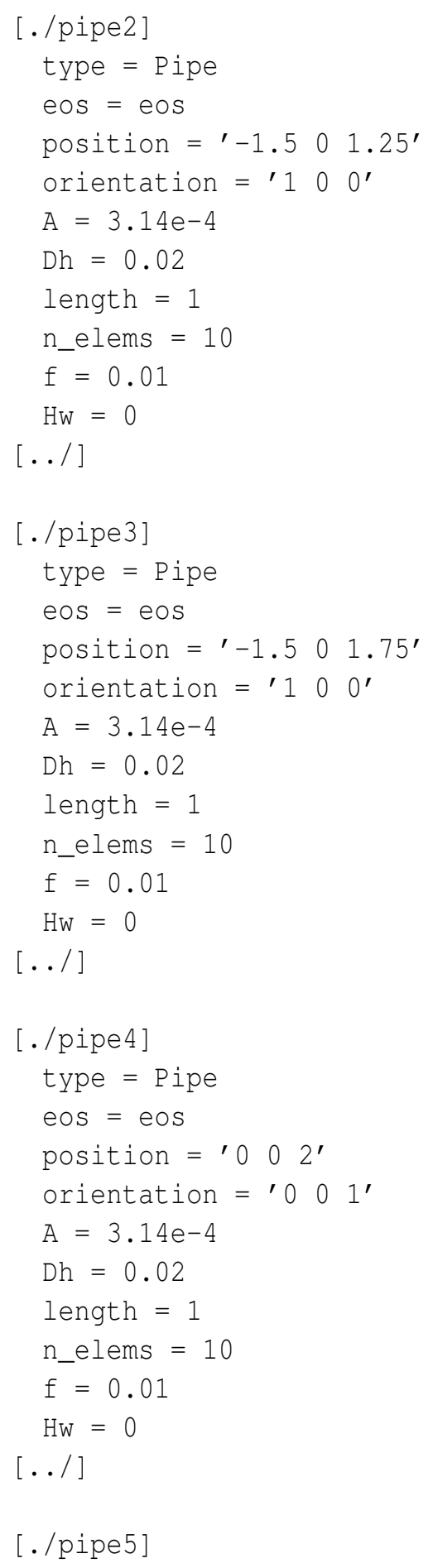




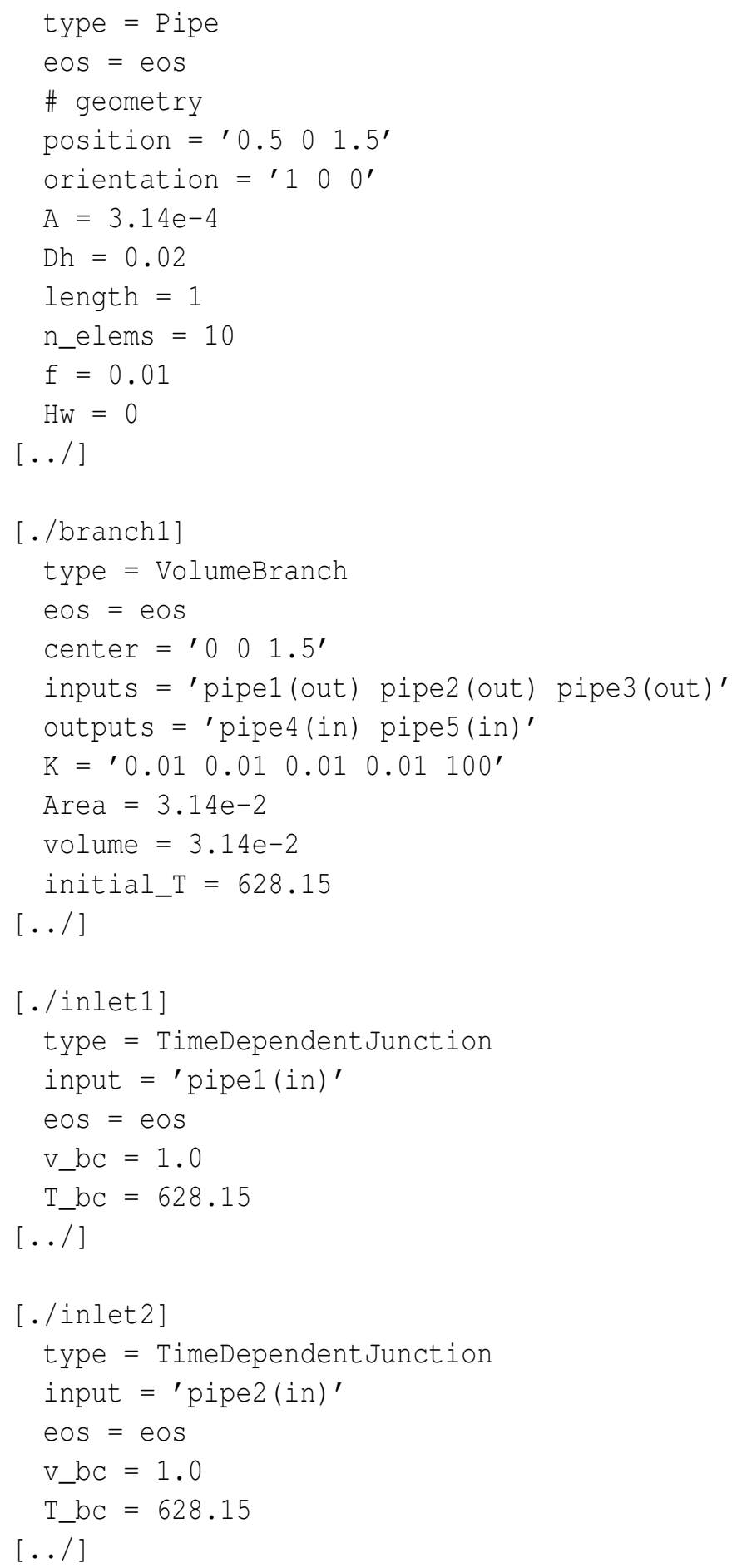




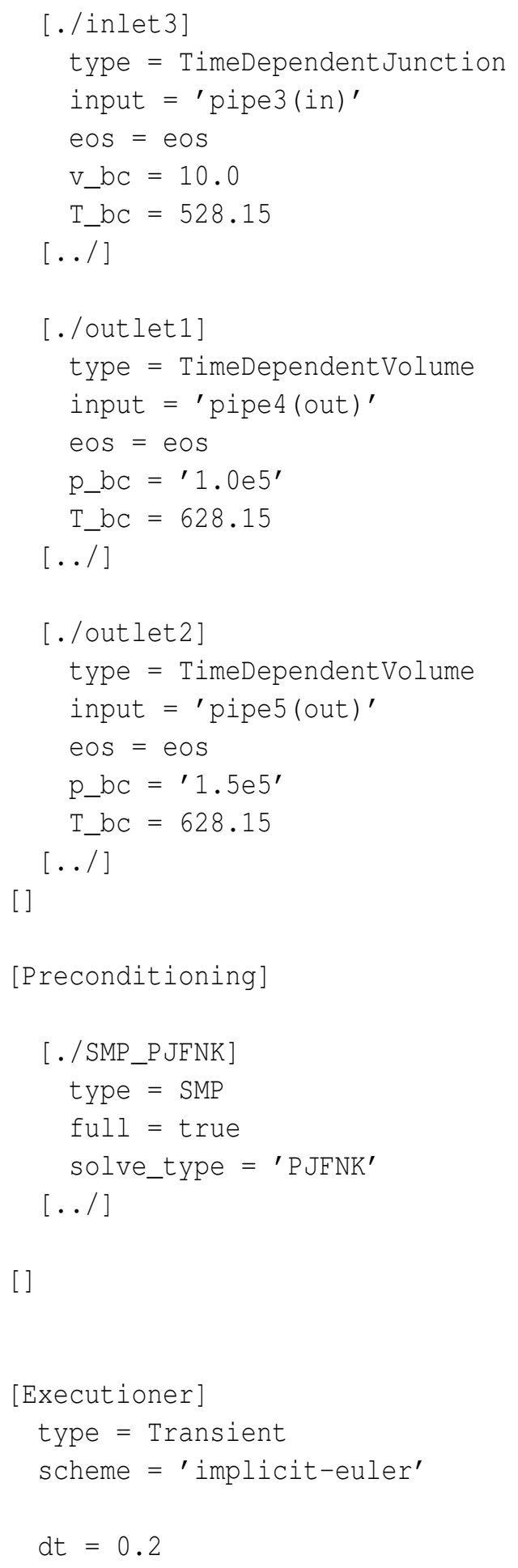




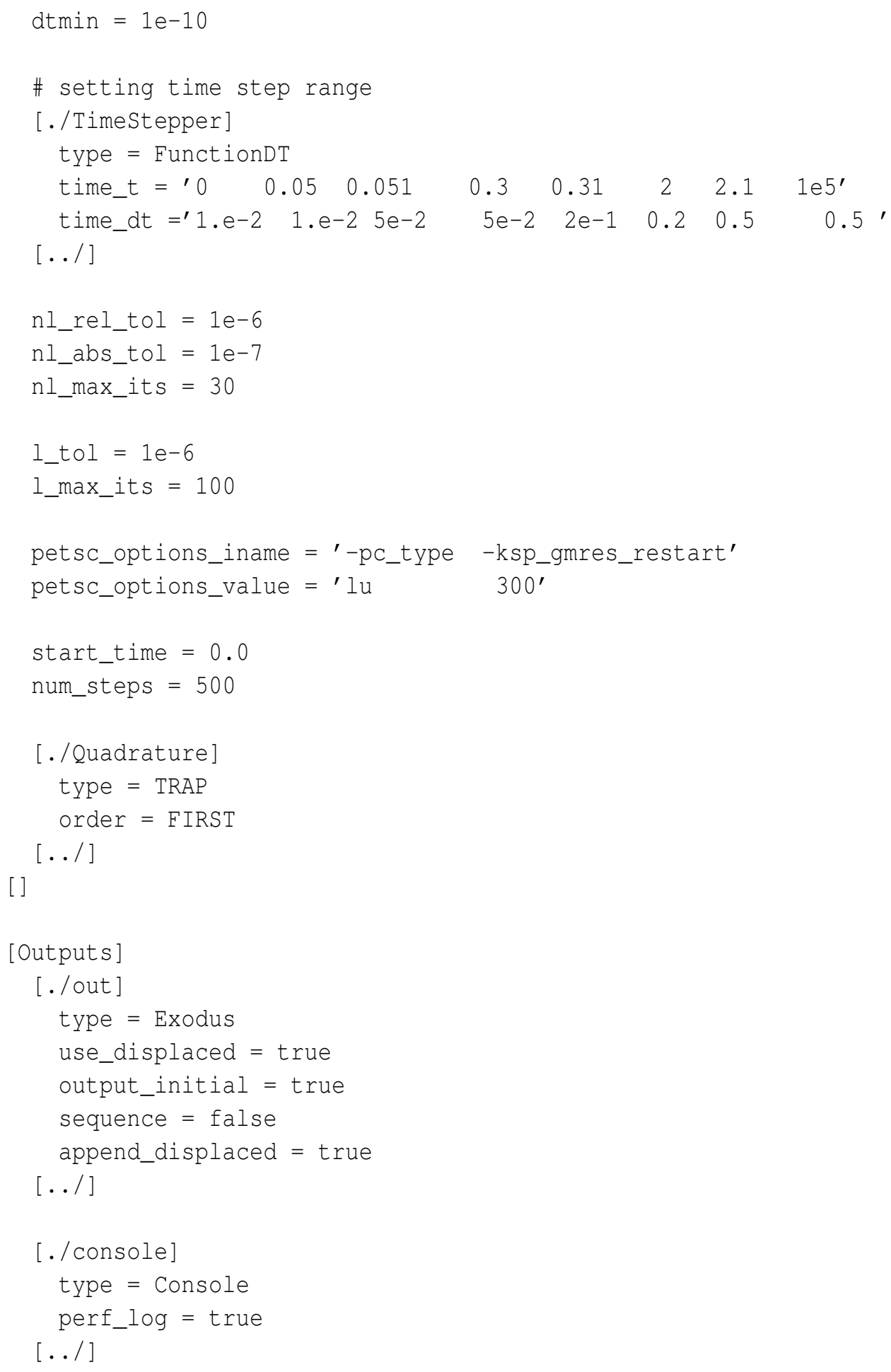




\subsubsection{Description of the Input File}

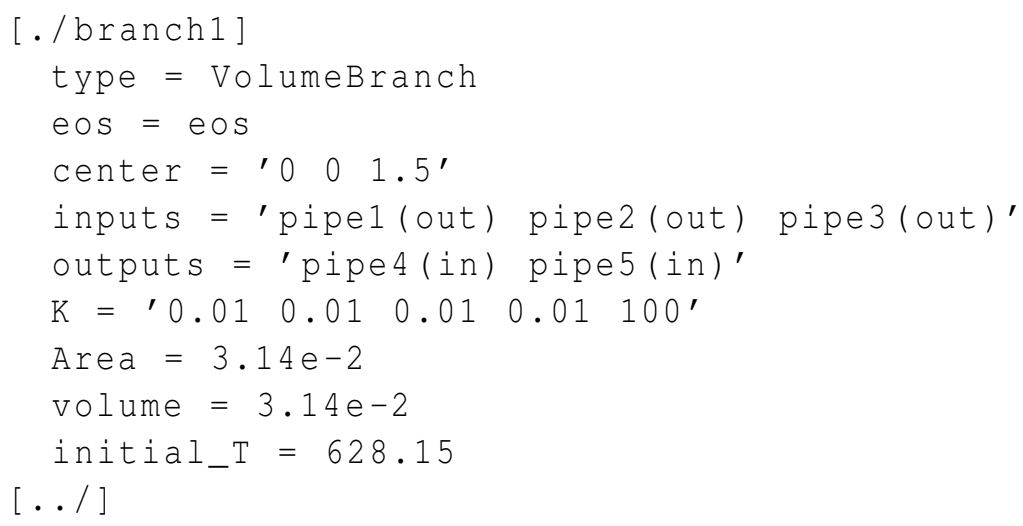

center The $(x, y, z)$ coordinate of the center of the volume branch.

volume The volume of VolumeBranch component.

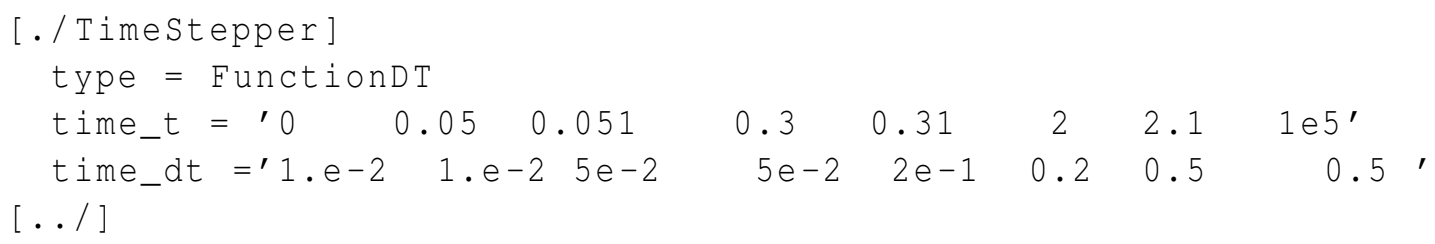

[./TimeStepper] Subblock to define time step size as a function of simulation time.

type $\quad$ FunctionDT type to be used.

time_t The value of time $t$.

time_dt The value of time step. 


\subsection{Example 8: A Simple Pipe Loop with Pump}

\subsubsection{Problem Description}

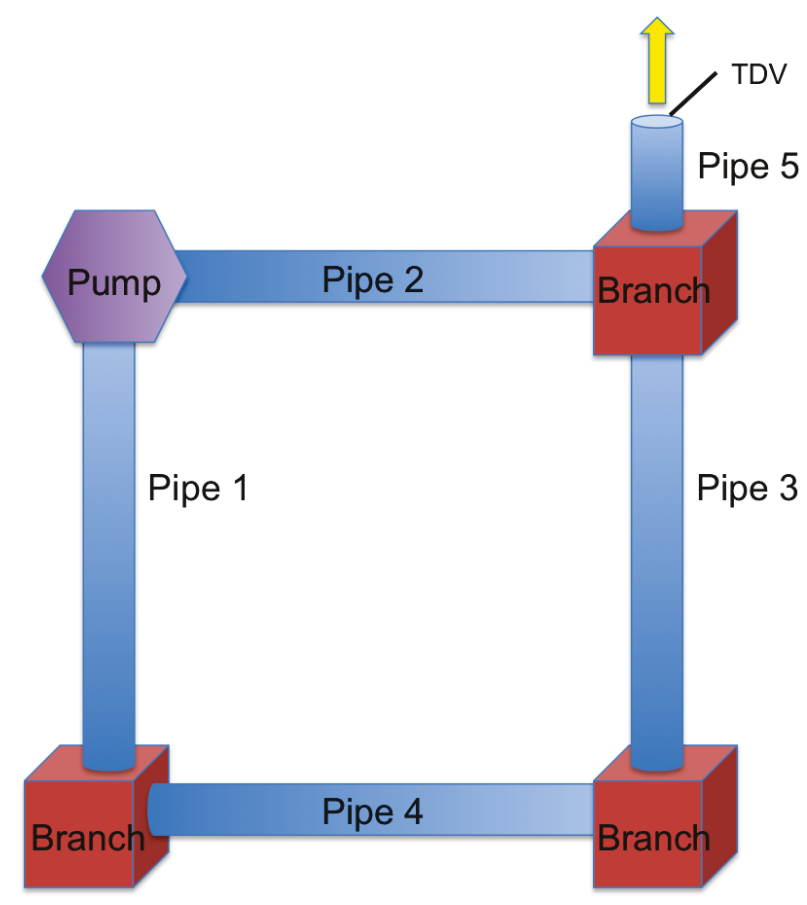

Figure 8. Diagram of a simple loop of pipes connected by branches and a pump

This example shows a loop made up of 5 pipes, 3 branches and a pump. The pump acts as a junction, connecting pipes 1 and 2, and pipe 5 is used as an outlet to help control the pressure. The input parameters used for the Pump model are the following:

Pump head $=1.0$

Form loss coefficient (in the reverse direction $)=$ '10. 10.'

Area $=0.785398163 e-3 m^{2}$

Initial pressure $=1 . \mathrm{e} 5 \mathrm{~Pa}$ 


\subsubsection{Input File}

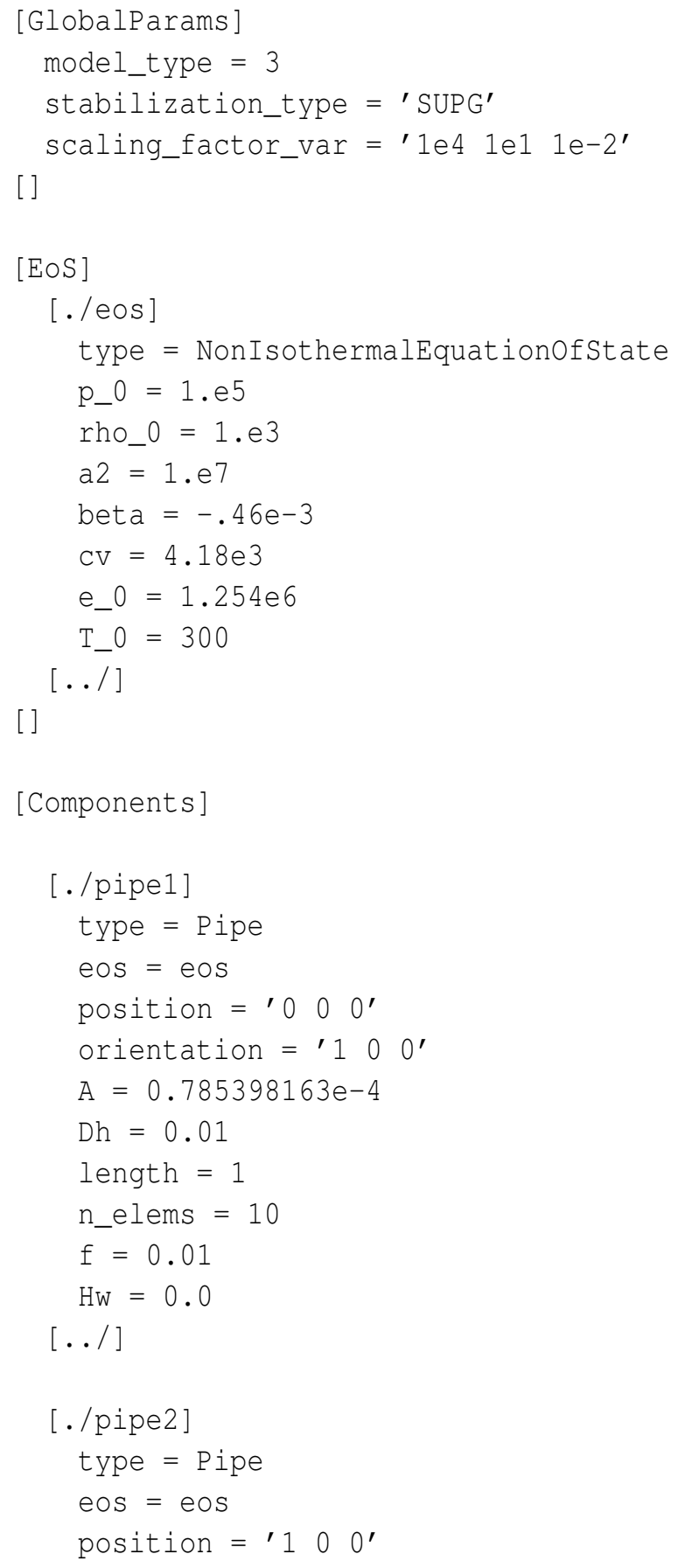




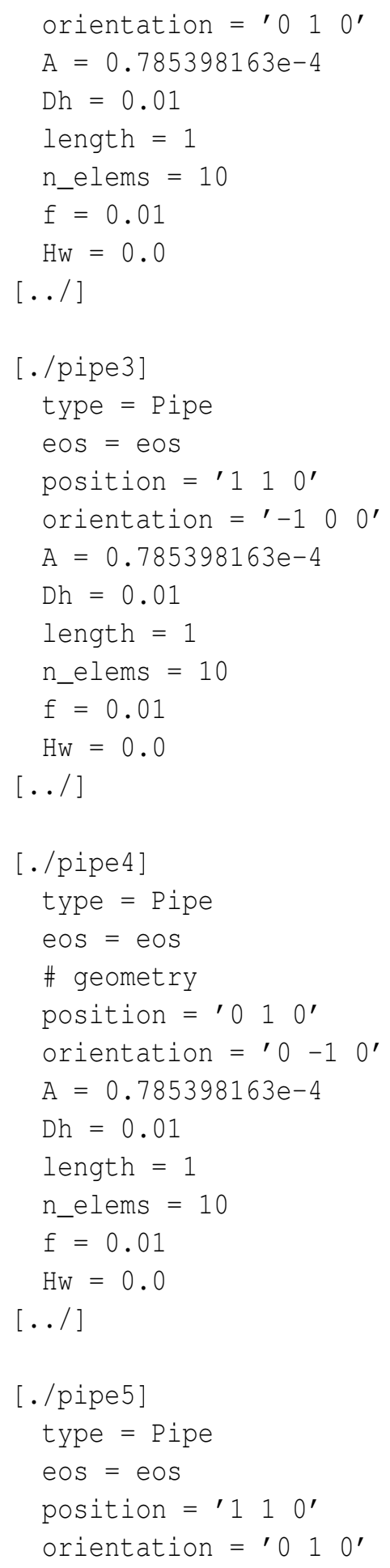




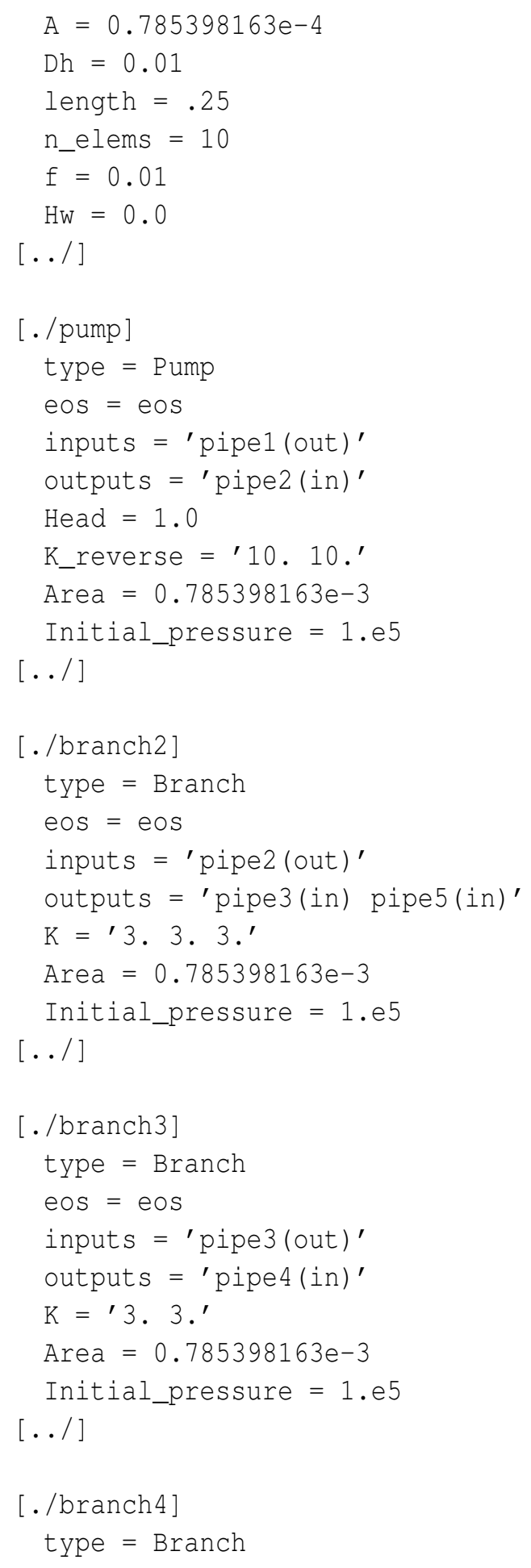




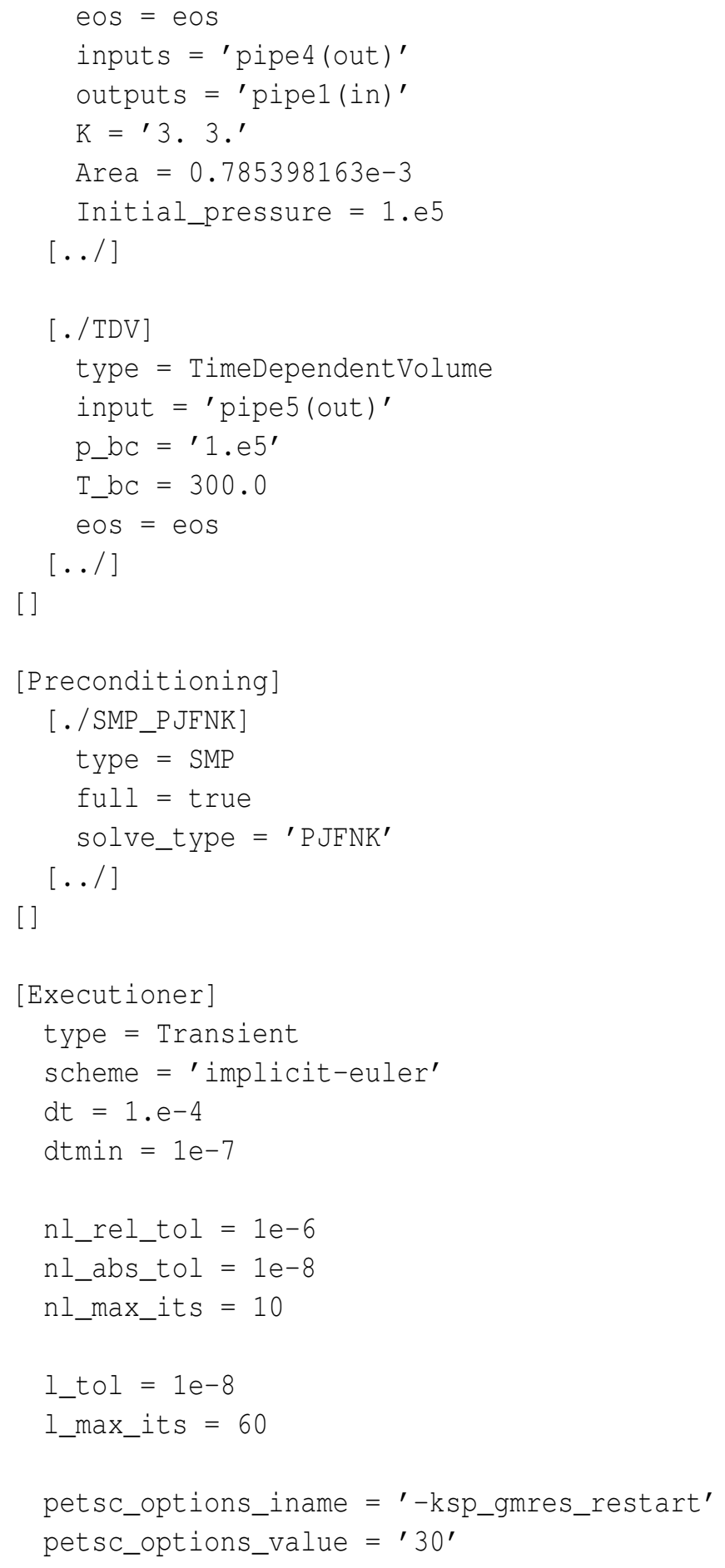




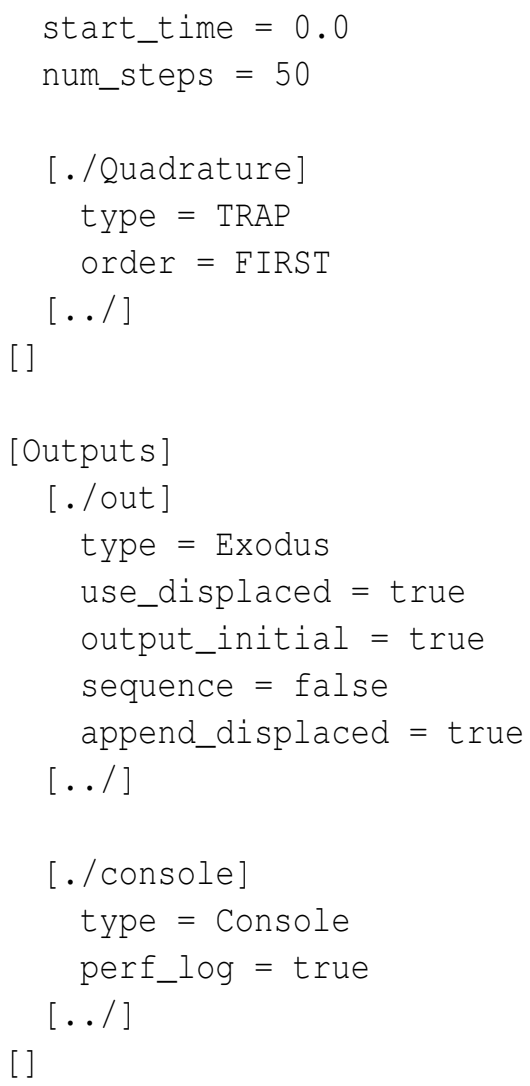

\subsubsection{Description of the Input File}

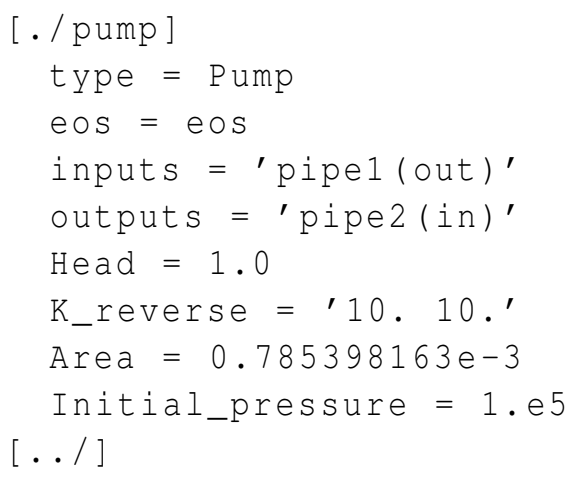

[./pump] Subblock for the pump component. 


Component type is a pump.
eos
Name of equation of state to be used by this pump component.
The inlet of the pump connects to outlet of pipe1.
outputs
Head




\subsection{Example 9: A Heat Exchanger Problem}

\subsubsection{Problem Description}

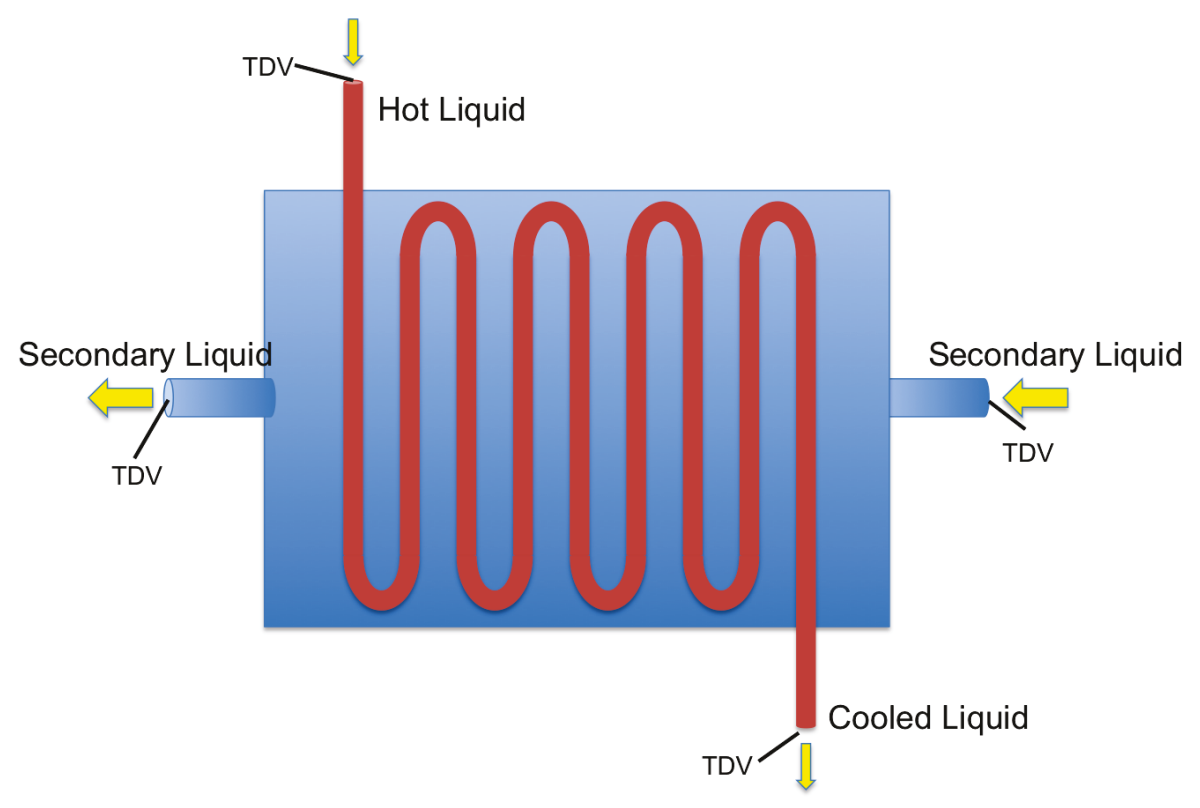

Figure 9. Diagram of a heat exchanger problem

This examples illustrates how to run a case with the heat exchanger component. The heat exchanger has two inlets and two outlets, primary and secondary respectively. The primary side inlet lets in hot water, while the secondary side inlet lets in liquid at a cooler temperature. This helps cool the primary side liquid, so the primary side outlet temperature is somewhat lower while the secondary side outlet temperature is somewhat higher. This example problem is for subcooled water only. The input parameters used for this case are listed in the following:

Materials:

Thermal Conductivity $=100.0$

Density $=100.0$

Specific Heat $=100.0$

Heat Exchanger: 
Area of primary pipe $=0.785398163 e-4 m^{2}$

Area of secondary pipe $=0.785398163 e-4 m^{2}$

Diameter of primary pipe $=0.01 \mathrm{~m}$

Diameter of secondary pipe $=0.01 \mathrm{~m}$

Length $=1 \mathrm{~m}$

Primary convective heat transfer coefficient $=1$.e4

Secondary convective heat transfer coefficient $=1$.e 4

Primary heat flux perimeter $=0.031415926520$

Secondary heat flux perimeter $=0.031415926520$

Friction coefficient $=0.01$

Initial wall temperature $=300.0 \mathrm{~K}$

Dimension of mesh used for wall $=1$

Thickness of the wall between primary and secondary loop $=0.001 \mathrm{~m}$

Primary inlet TDV:

Pressure $=1.05 \mathrm{e} 5 \mathrm{~Pa}$

Temperature $=400.0 \mathrm{~K}$

Primary outlet TDV:

Pressure $=1 . \mathrm{e} 5 \mathrm{~Pa}$

Temperature $=300.0 \mathrm{~K}$

Secondary inlet TDV:

Pressure $=1.05 \mathrm{e} 5 \mathrm{~Pa}$

Temperature $=300.0 \mathrm{~K}$

Secondary outlet TDV:

Pressure $=1 . e 5 \mathrm{~Pa}$

Temperature $=300.0 \mathrm{~K}$

\title{
5.9.2 Input File
}

\author{
[GlobalParams] \\ global_init_P $=1 . e 5$
}




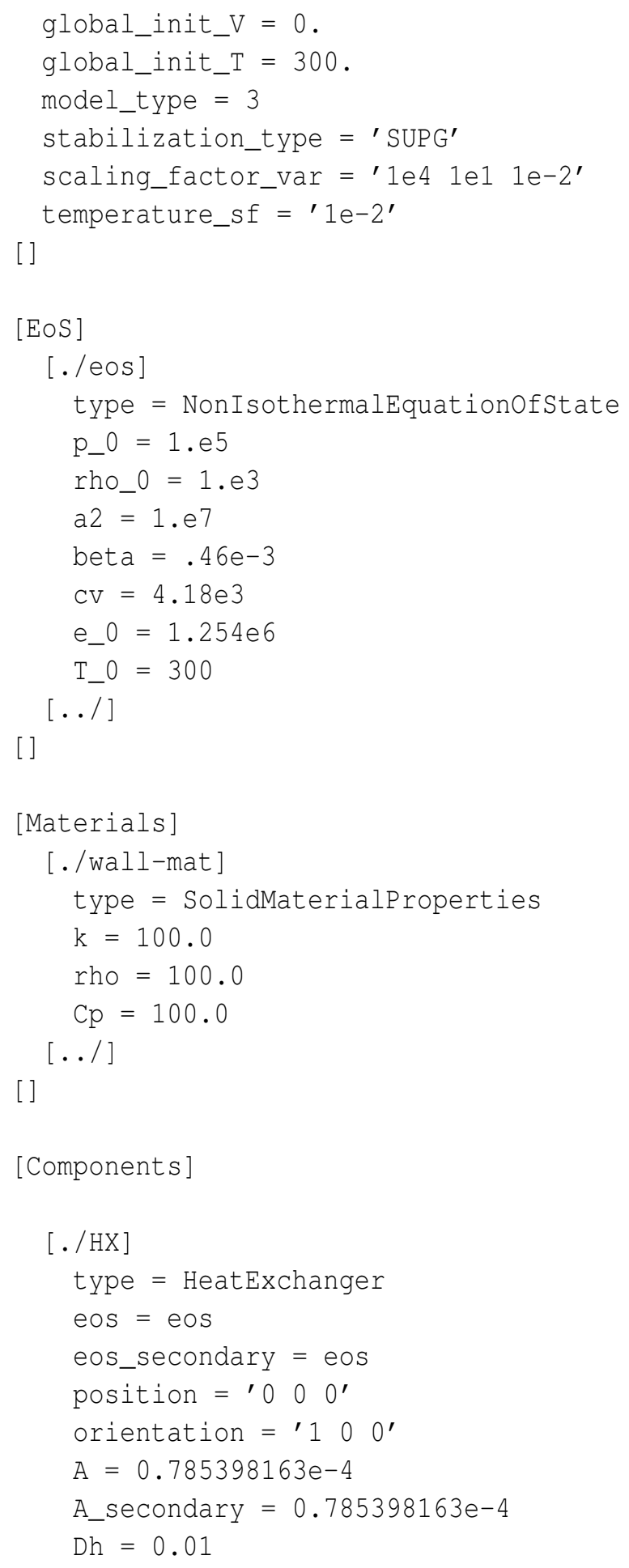




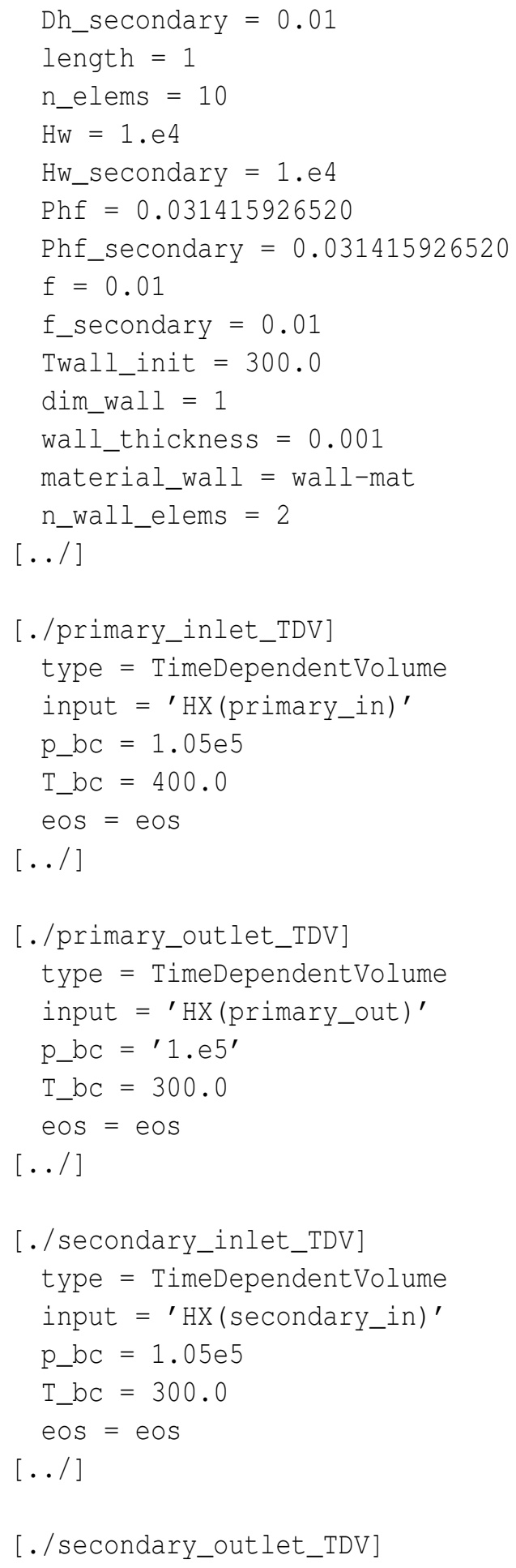




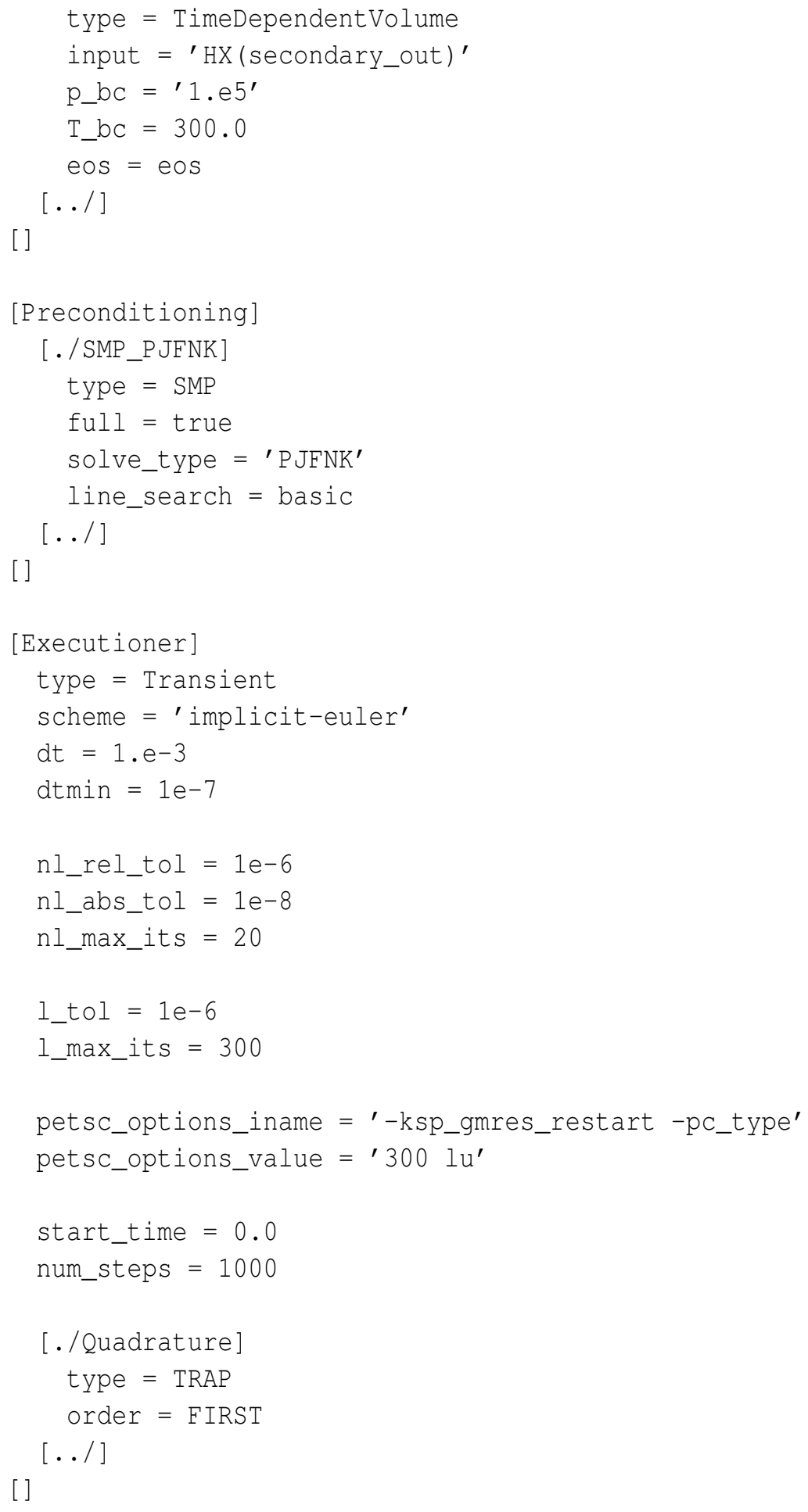




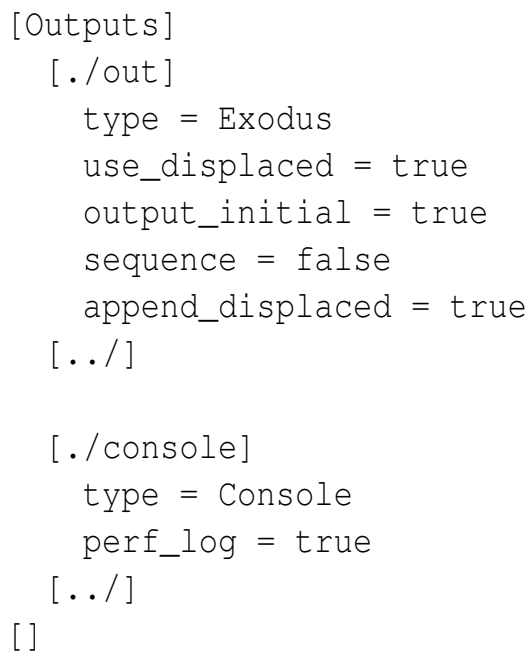

\subsubsection{Description of the Input File}

The input parameters for the heat exchanger component needs explanantions.

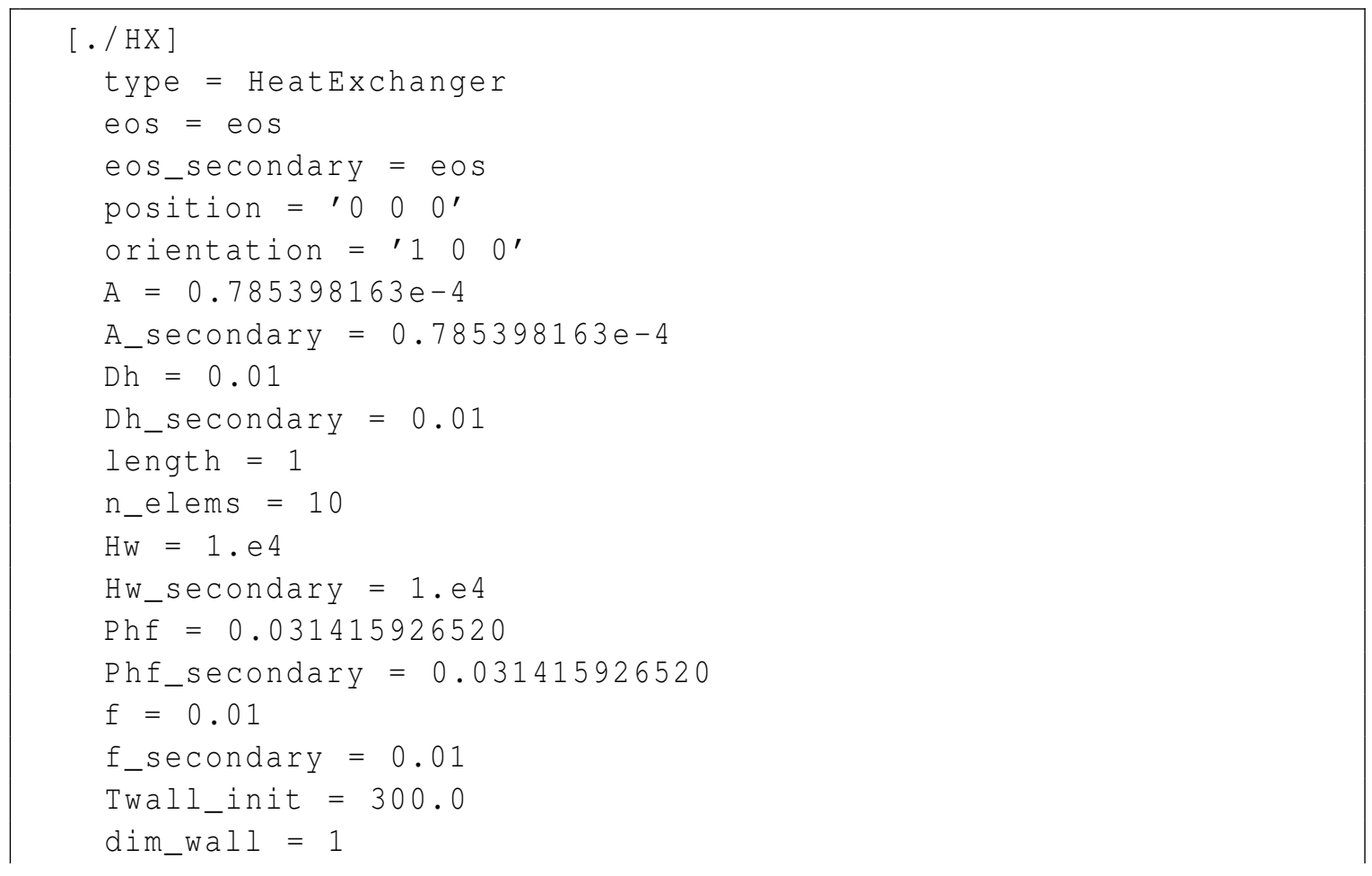




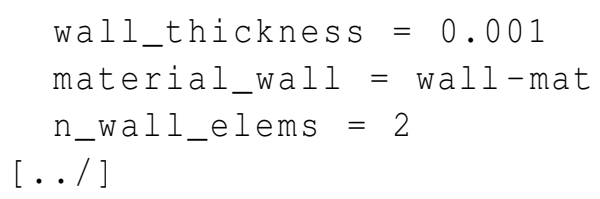

[./HX]

eos

eos_secondary

position

orientation

A

A_secondary

$\mathrm{Dh}$

Dh_secondary

length

n_elems

$\mathrm{HW}$

hw_secondary

Phf

Phf_secondary

f
Subblock for the heat exchanger component.

Name of the equation of state to be used by the fluids in the primary side of the heat exchanger.

Name of the equation of state to be used by the fluids in the secondary side of the heat exchanger.

The $x, y, z$ coordinate of the start of heat exchanger.

The orientation vector of heat exchanger.

The flow area of the primary side of the heat exchanger.

The flow area of the secondary side of the heat exchanger.

Hydraulic dimater of the primary side of the heat exchanger.

Hydraulic diameter of the secondary side of the heat exchanger.

Length of heat exchanger.

The number of elements in the heat exchanger.

The convective heat transfer coefficient on the primary side of the heat exchanger.

The convective heat transfer coefficient on the secondary side of the heat exchanger.

Heat flux perimeter of the primary side of the heat exchanger.

Heat fluc perimeter of the secondary side of the heat exchanger.

Wall friction coefficient on the primary side of the heat exhanger. 
f_secondary Wall friction ceofficient on the secondary side of the heat exchanger.

Twall_init Prescribed initial temperature of the solid materials between the fluids.

dim_wall The dimension of the mesh used in the heat conduction calculations of the wall in the heat exchanger.

wall_thickness The wall thickness.

material_wall Name of the material used in the wall.

n_wall_elems Number of elements in the wall. 


\subsection{Example 10: A Loop With Core Channel and Heat Exchanger}

\subsubsection{Problem Description}

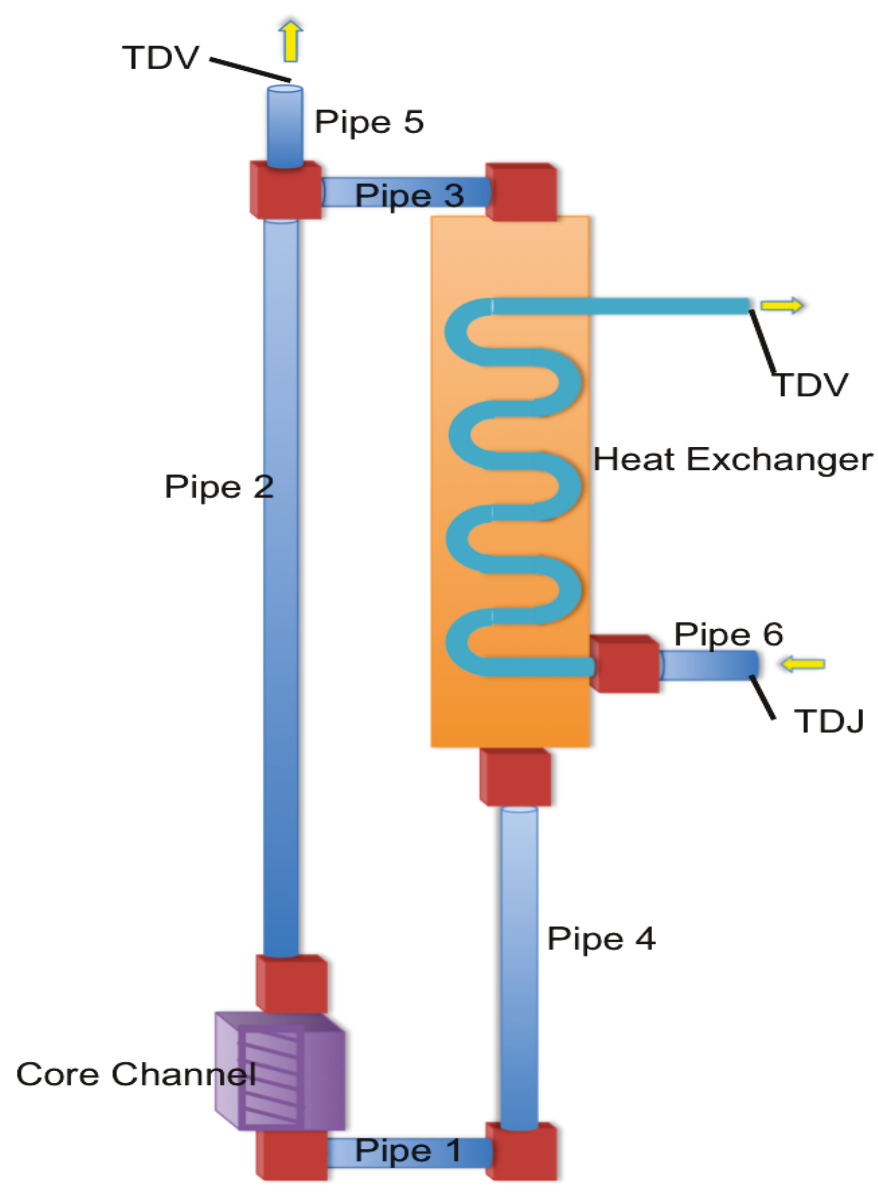

Figure 10. Diagram of a loop with core channel and heat exchanger

This example is a simple loop whcich consists of 6 pipes, a core channel, and a heat exchanger. Each component differs in length, but has the same area and diameter. The core channel is used to heat up the liquid, while the heat exchanger is used to cool it down. Pipe 5 is used to control the system pressure. The input parameters are listed in the following: 
Materials:

Fuel

Thermal conductivity $=29.3 \mathrm{~W} / \mathrm{m} \mathrm{K}$

Specific heat $=191.67 \mathrm{~J} / \mathrm{kg} \mathrm{K}$

Density $=1.4583 \mathrm{e} 4 \mathrm{~kg} / \mathrm{m}^{3}$

Clad

Thermal conductivity $=26.3 \mathrm{~W} / \mathrm{m} \mathrm{K}$

Specific heat $=638 \mathrm{~J} / \mathrm{kg} \mathrm{K}$

Density $=7.646 e 3 \mathrm{~kg} / \mathrm{m}^{3}$

Wall

Thermal conductivity $=26.3 \mathrm{~W} / \mathrm{m} \mathrm{K}$

Specific heat $=638 \mathrm{~J} / \mathrm{kg} \mathrm{K}$

Density $=7.646 \mathrm{e} 3 \mathrm{~kg} / \mathrm{m}^{3}$

Reactor's initial power $=5.129 \mathrm{e} 7$

Pipe1:

Area $=0.44934 m^{2}$

Diameter $=2.972 \mathrm{e}-3 \mathrm{~m}$

Length $=1 \mathrm{~m}$

Friction Coefficient $=0.001$

$\mathrm{Hw}=0.0$

Pipe 2 length $=5.18 \mathrm{~m}$

Pipe 3 length $=1 \mathrm{~m}$

Pipe 4 length $=2.27 \mathrm{~m}$

Pipe 5 length $=0.02 \mathrm{~m}$

Pipe 6 length $=0.3 \mathrm{~m}$

Core Channel:

Length $=0.8 \mathrm{~m}$

Friction coefficient $=0.022$

Convective heat transfer coefficient $=1.6129 \mathrm{e} 5$

Heat flux perimeter $=497.778$ 
Initial solid temperature $=628.15 \mathrm{~K}$

Width of each heat structure $=$ '0.00348 0.00052'

Heat Exchanger:

Area of primary pipe $=0.44934 \mathrm{~m}^{2}$

Area of secondary pipe $=0.44934 \mathrm{~m}^{2}$

Diameter of primary pipe $=0.0186 \mathrm{~m}$

Diameter of secondary pipe $=0.014 \mathrm{~m}$

Length $=3.71 \mathrm{~m}$

Primary convective heat transfer coefficient $=1.6129 \mathrm{e} 5$

Secondary convective heat transfer coefficient $=1.6129 \mathrm{e} 5$

Primary heat flux perimeter $=327.568$

Secondary heat flux perimeter $=327.568$

Friction coefficient $=0.022$

Initial wall temperature $=628.15 \mathrm{~K}$

Dimension of mesh used for wall $=1$

Thickness of the wall between primary and secondary loop $=0.0044 \mathrm{~m}$

Branches:

Form loss coefficients $=$ ' 0.50 .5 '

Area $=0.44934 m^{2}$

Initial Pressure $=1$ e $5 \mathrm{~Pa}($ Branch $1=2 \mathrm{e} 5 \mathrm{~Pa})$

Inlet:

Type $=$ TimeDependentJunction

Input = 'pipe6(in)'

Velocity $=0.5 \mathrm{~m} / \mathrm{s}$

Temperature $=628.15 \mathrm{~K}$

Outlet 1:

Type $=$ TimeDependent Volume

Input = 'IHX(secondary_out)'

Pressure $=1.0 \mathrm{e} 5 \mathrm{~Pa}$

Temperature $=761.15 \mathrm{~K}$

Outlet 2:

Type $=$ TimeDependentVolume

Input = 'pipe5(out)' Pressure $=1 \mathrm{e} 5 \mathrm{~Pa}$ 
Temperature $=783.15 \mathrm{~K}$

\subsubsection{Input File}

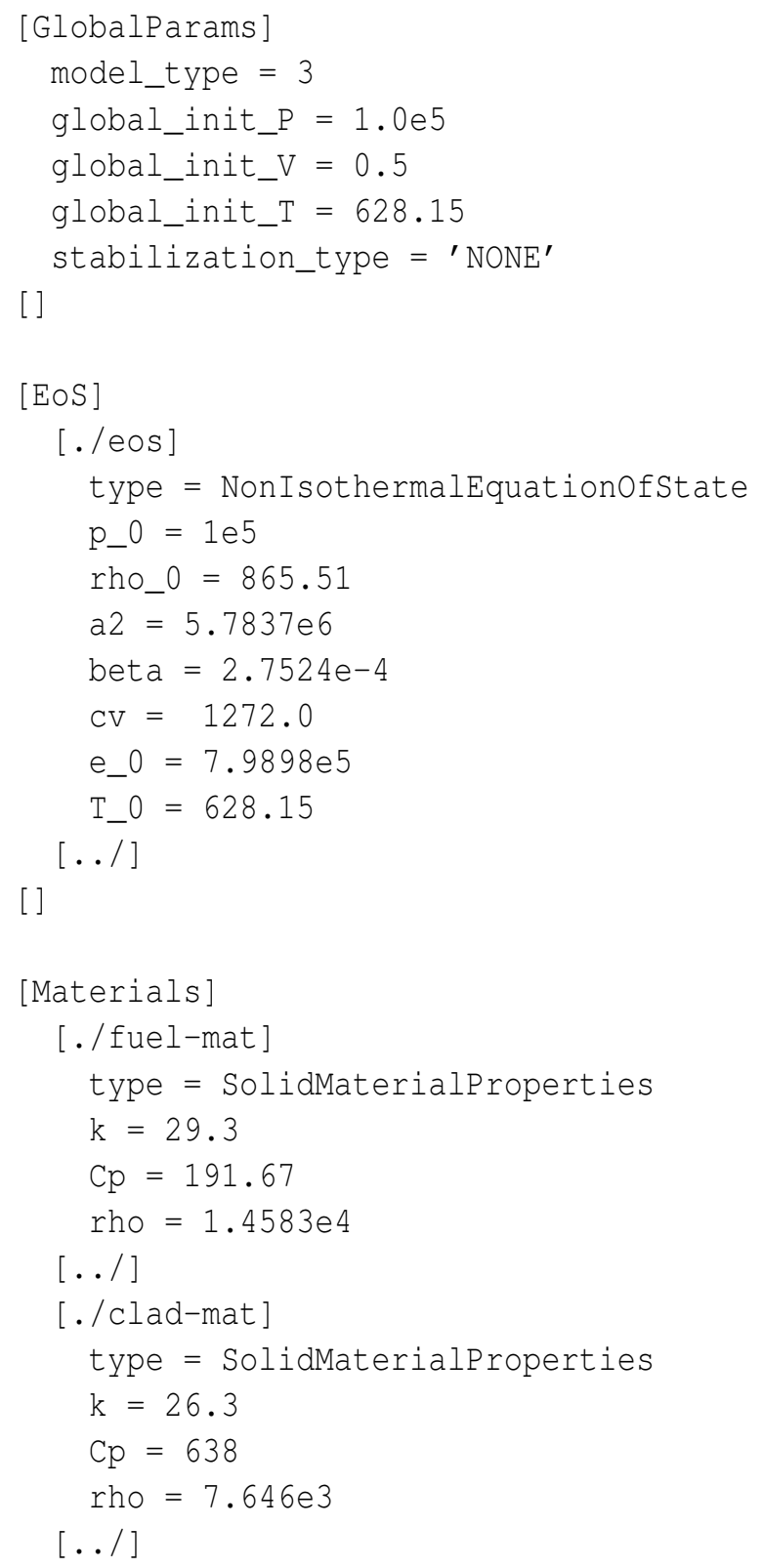




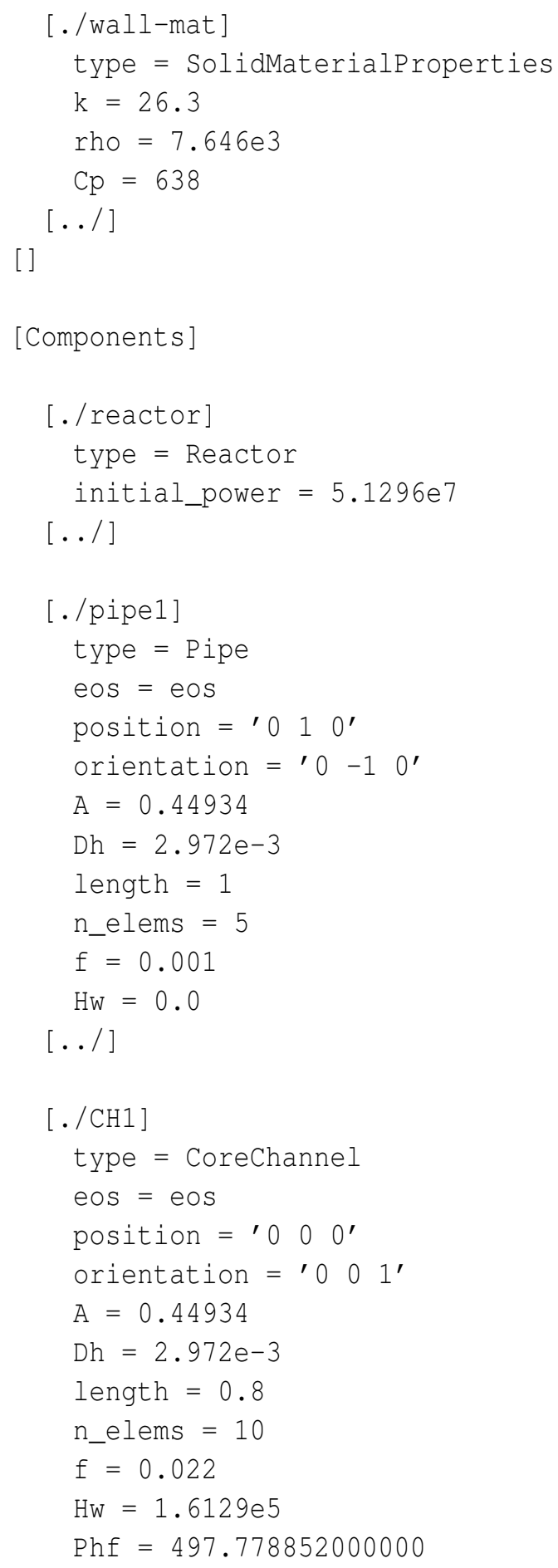




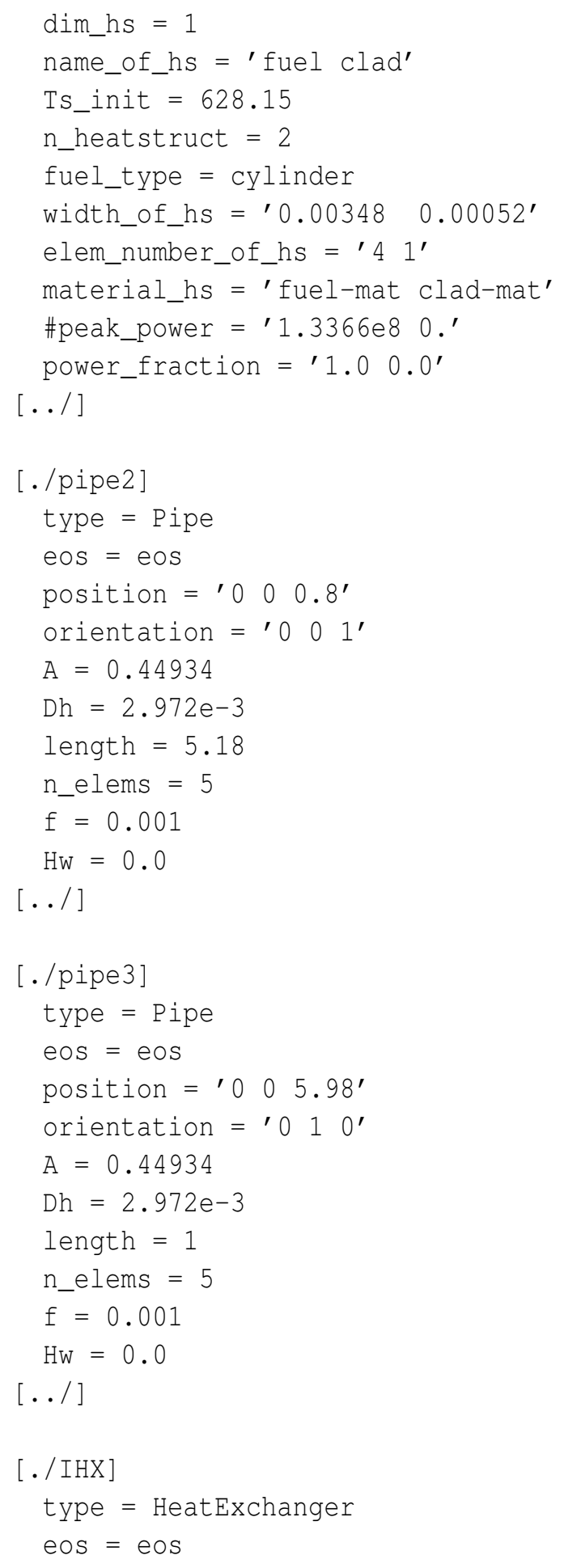




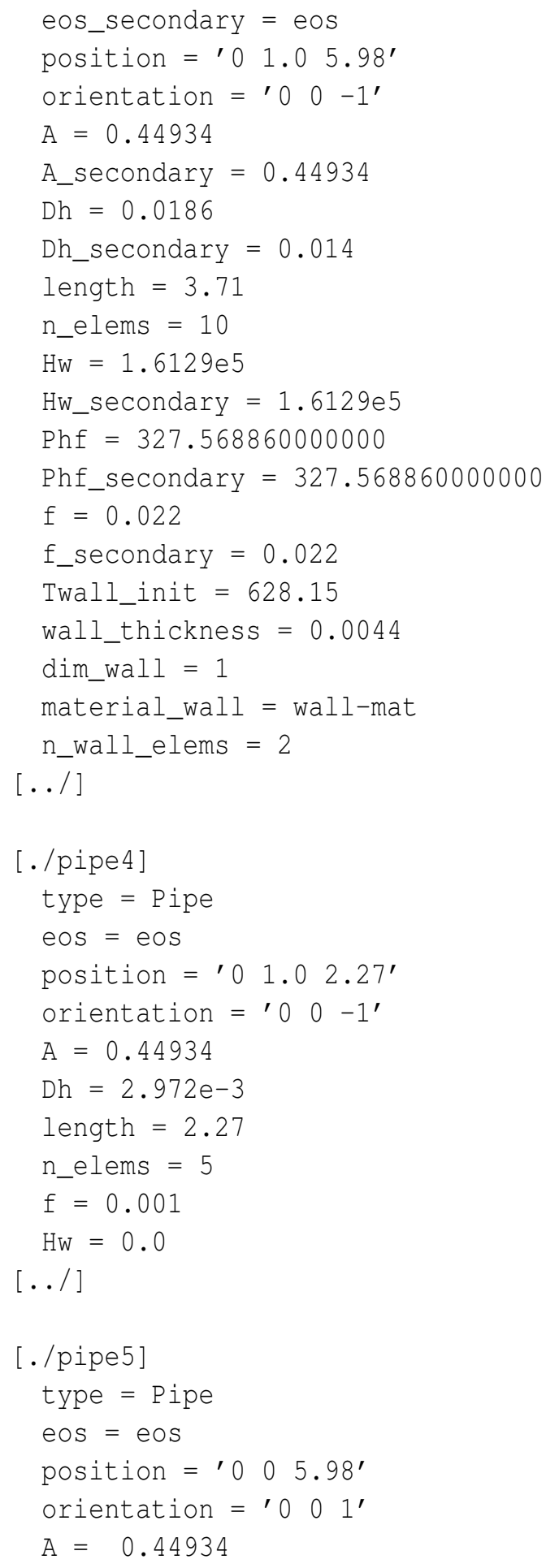




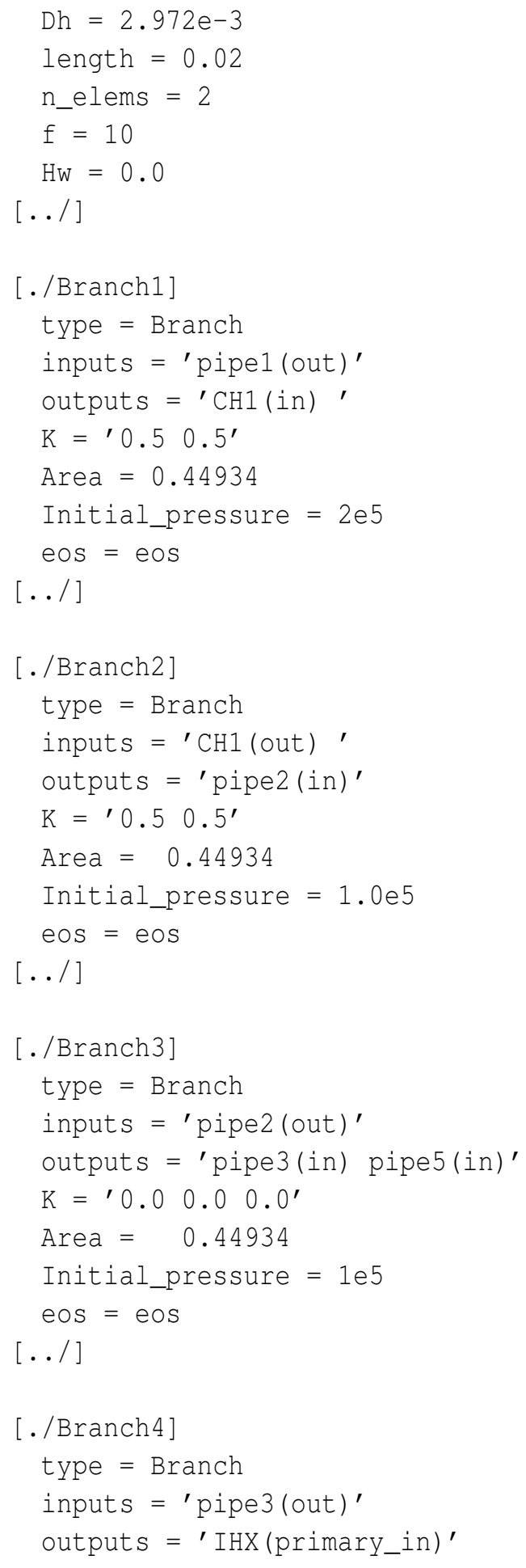




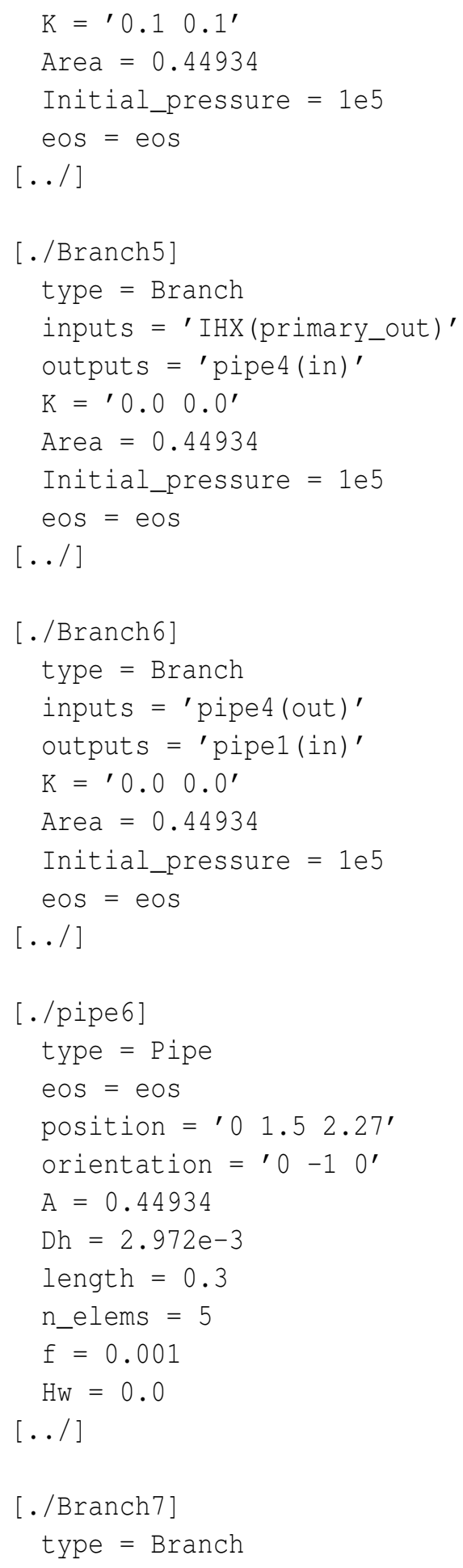




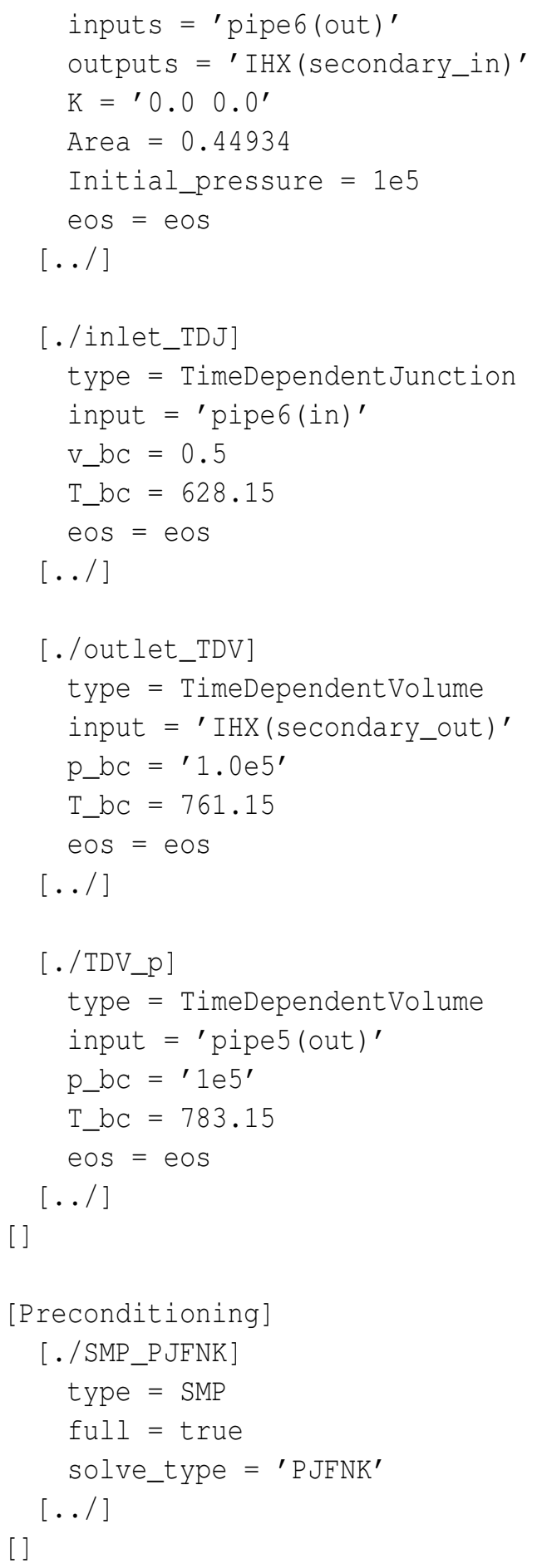




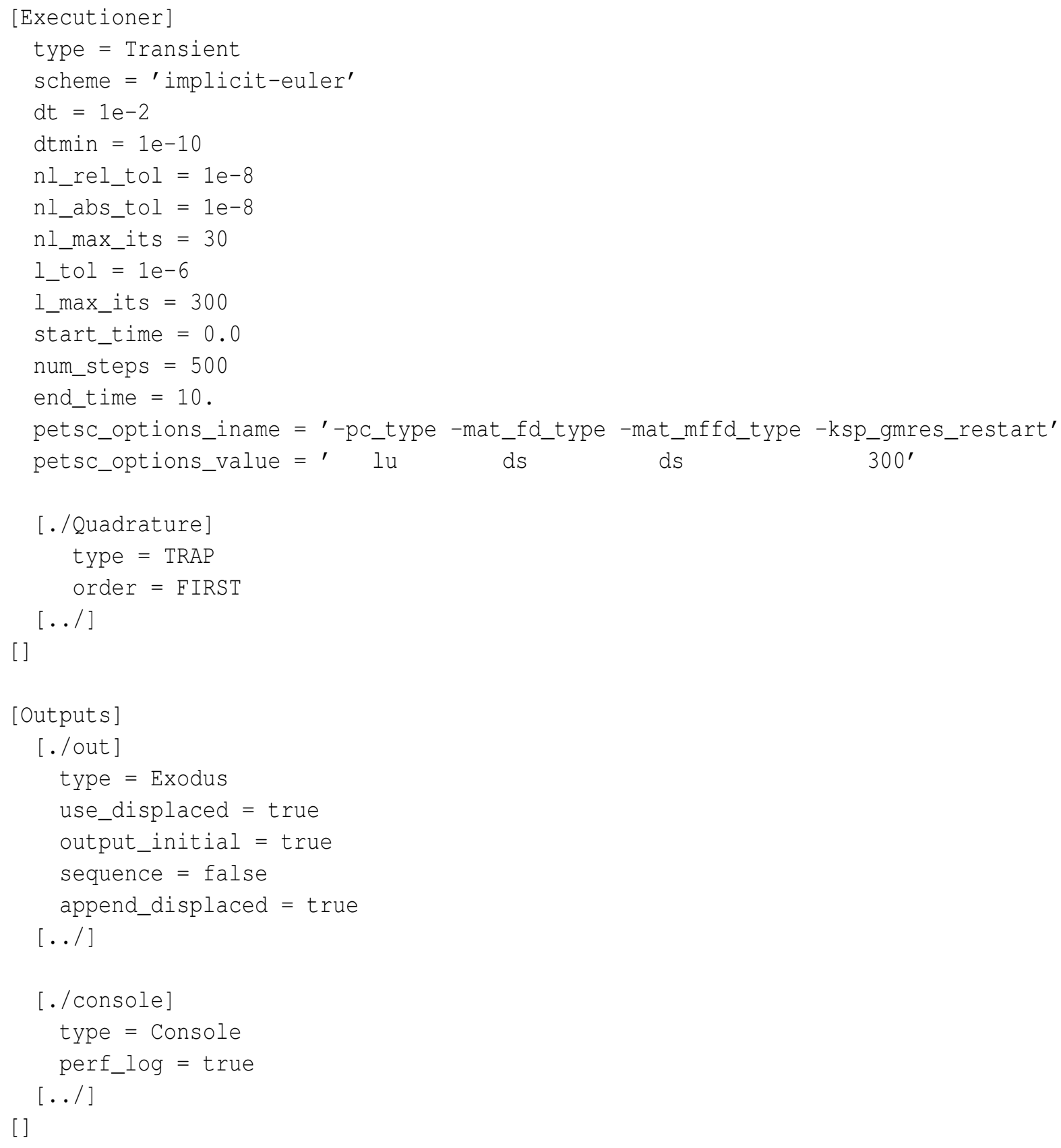




\subsection{Example 11: A Model Pressurized Water Reactor Problem}

\subsubsection{Problem Description}

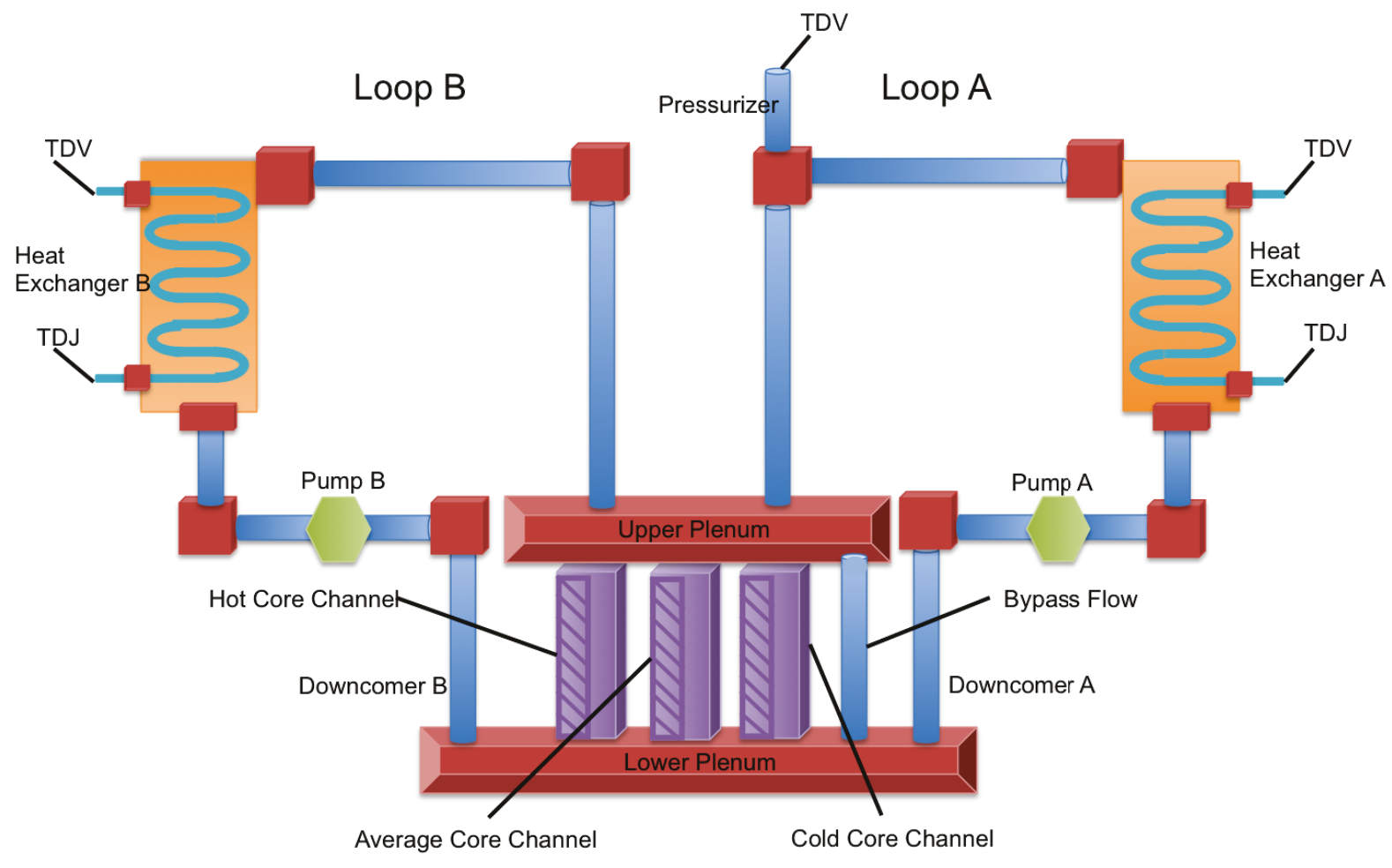

Figure 11. Diagram of a model pressurized water reactor problem

This example shows a simplified PWR plant model. It is made up of thress sections - Loop A, Loop B and a reator vessel model. The reactor vessel region contains three parallel core channels, a bypass flow channel, and an upper and lower plenum. The three core channels represent all the cooling channels and fuel rods in the high power region, average power region and low power region of the reactor core respectively. The upper and lower plenum are modeled with volume branches. The two loops have a Hot Leg, a Heat Exchanger and its secondary side pipes, the Cold Leg and a primary Pump. The heat exchanger secondary side pipes are modeled with subcooled water since the steam generator model is not available yet. Loop A contains a time dependent volume component that works as a pressurizer to help regulate the system pressure. 


\subsubsection{Input File}

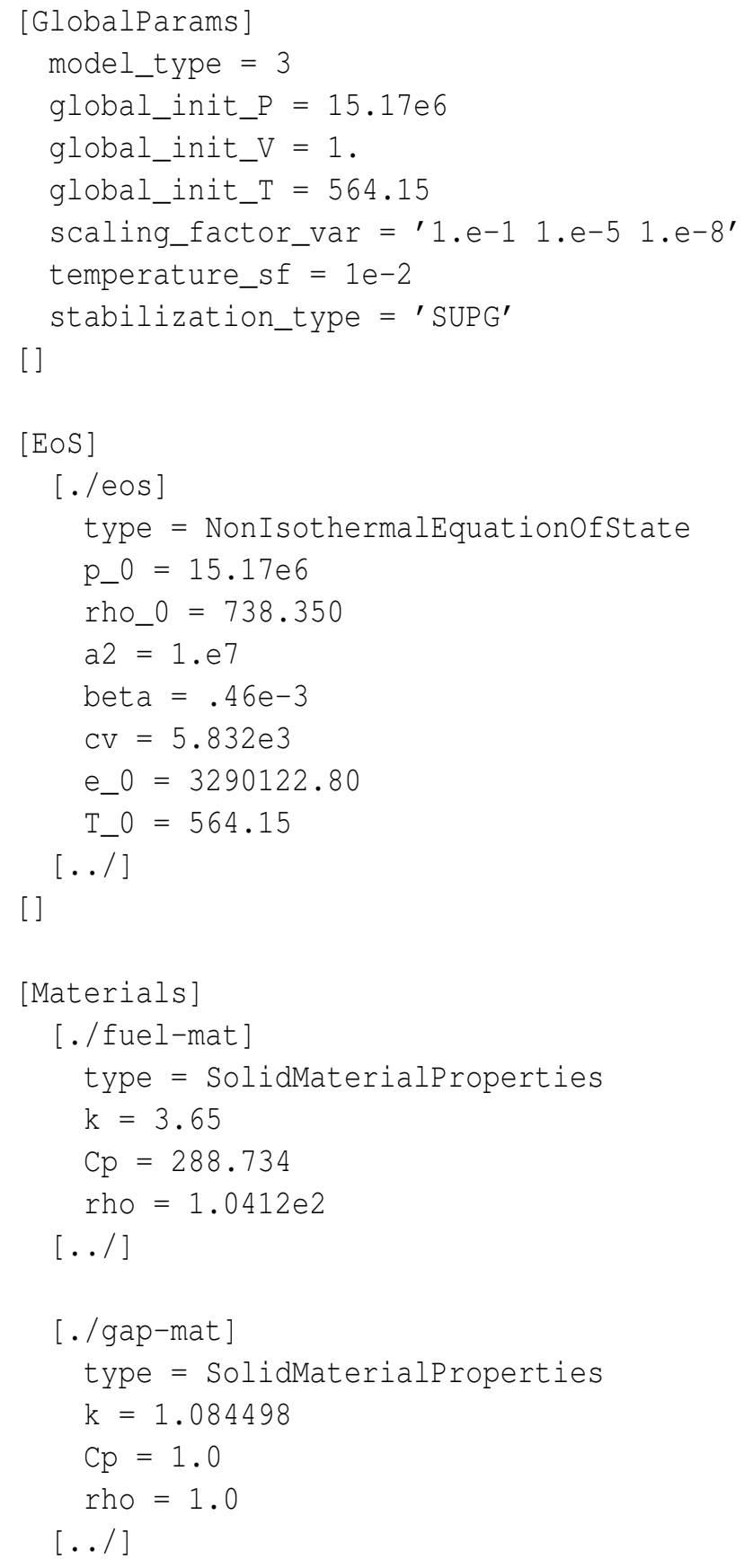




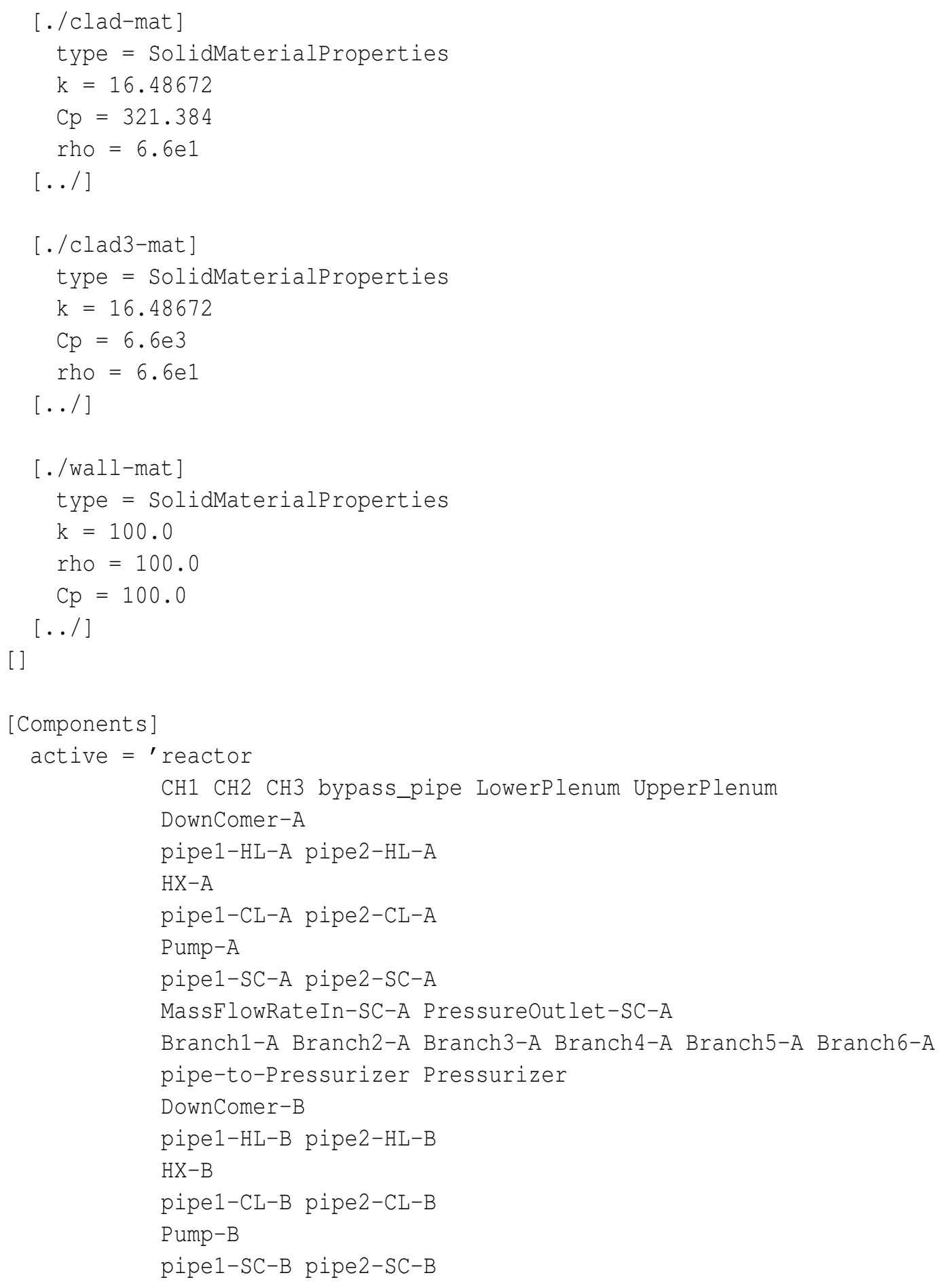




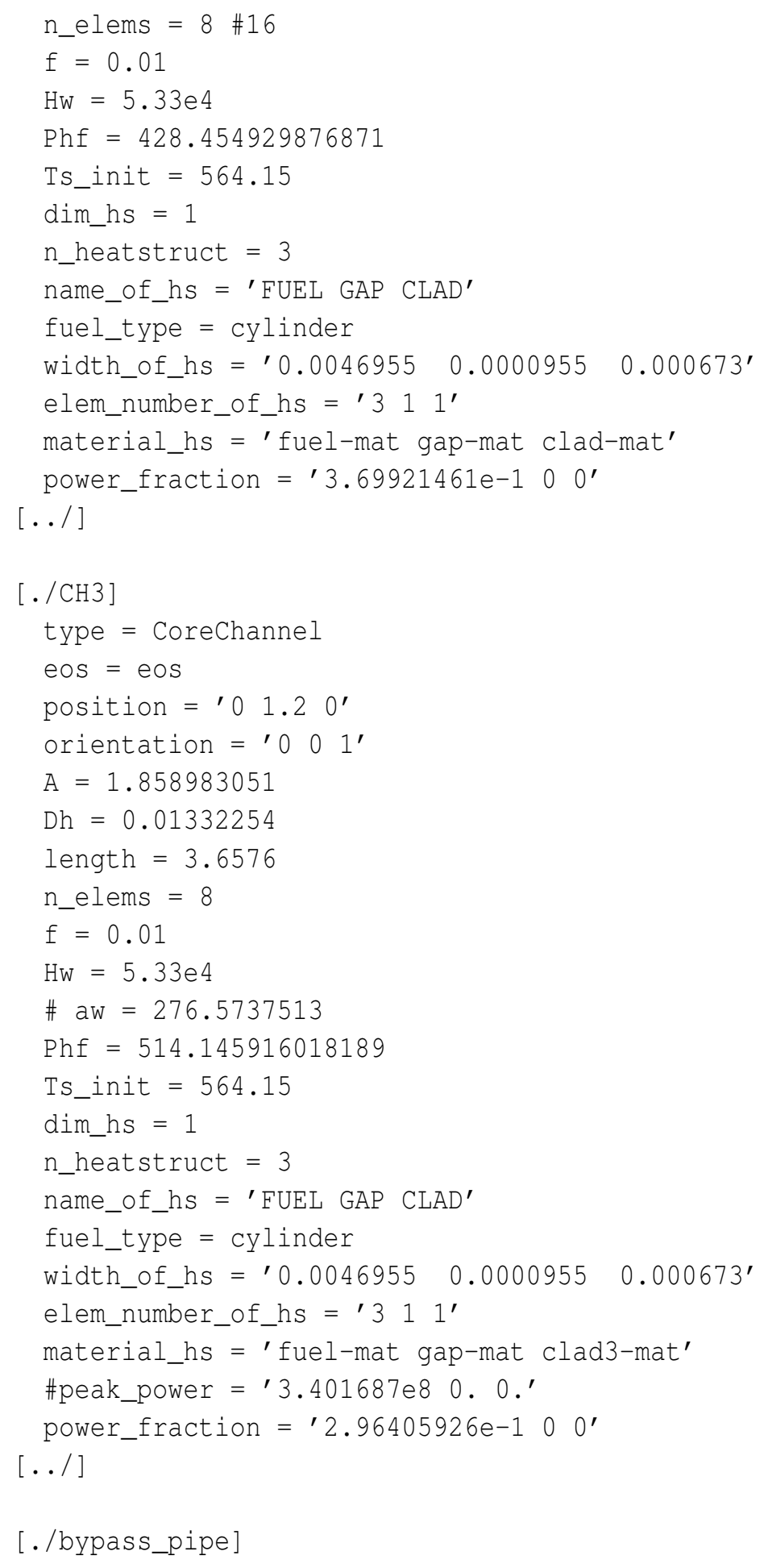




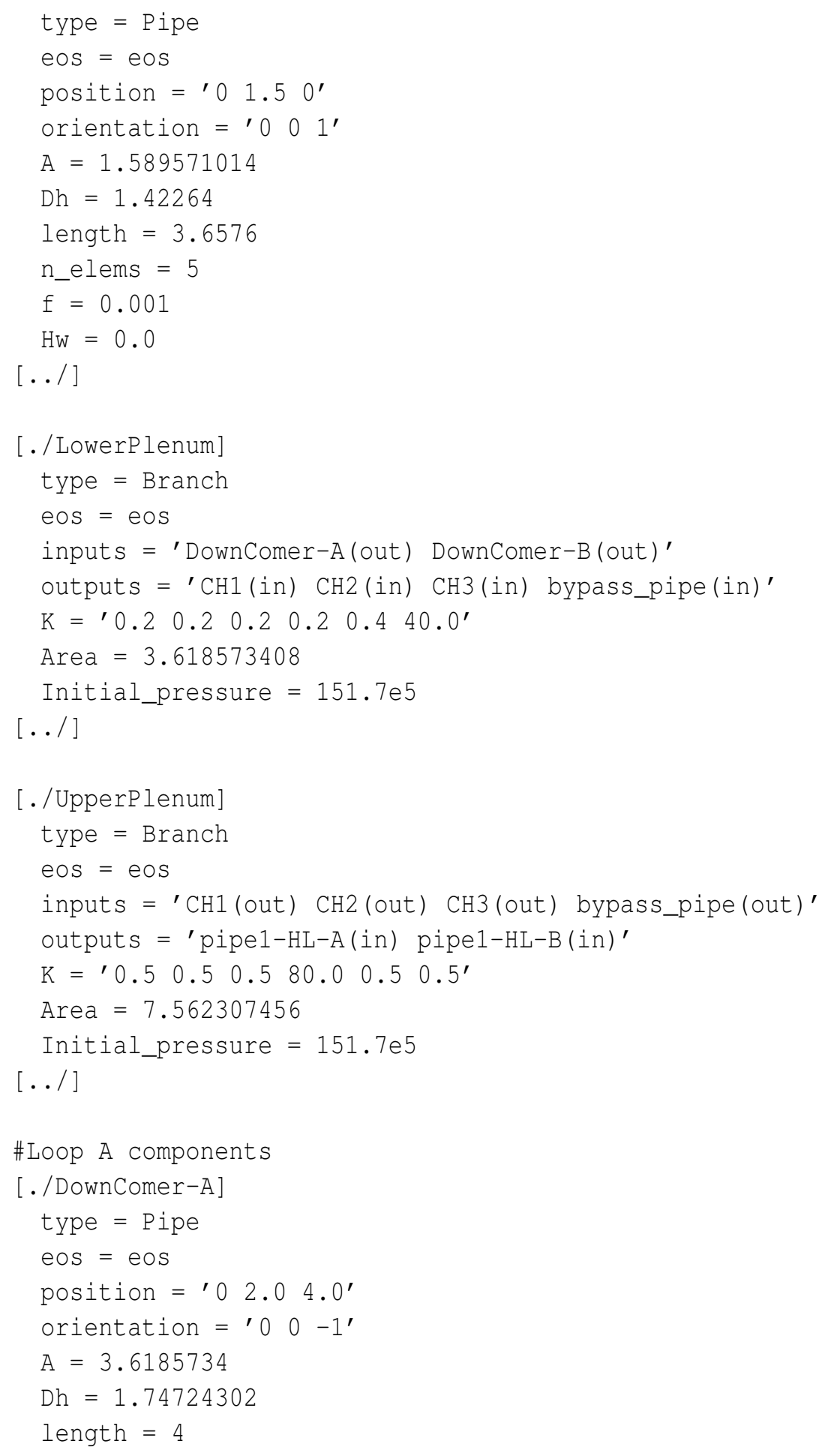




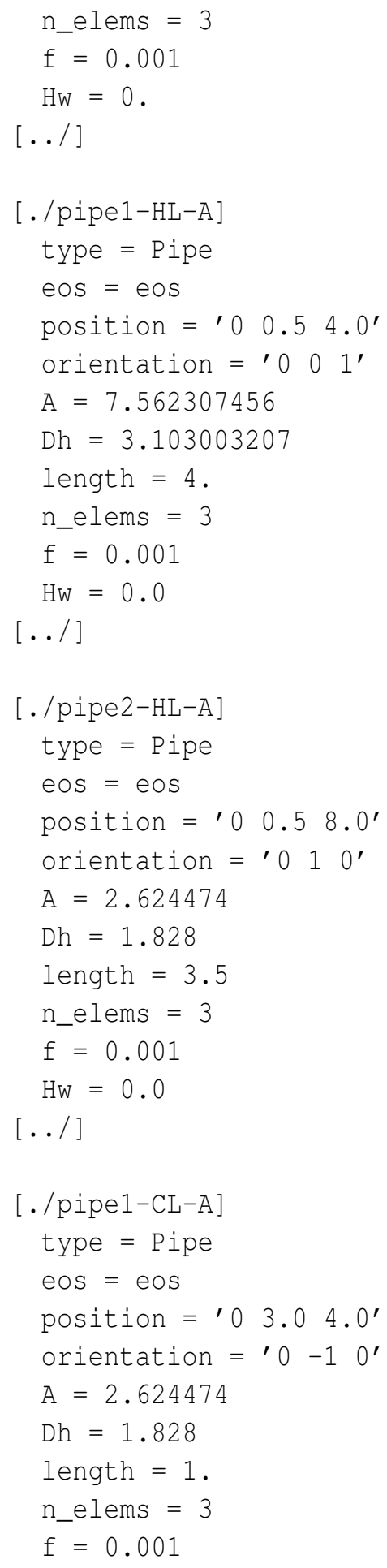




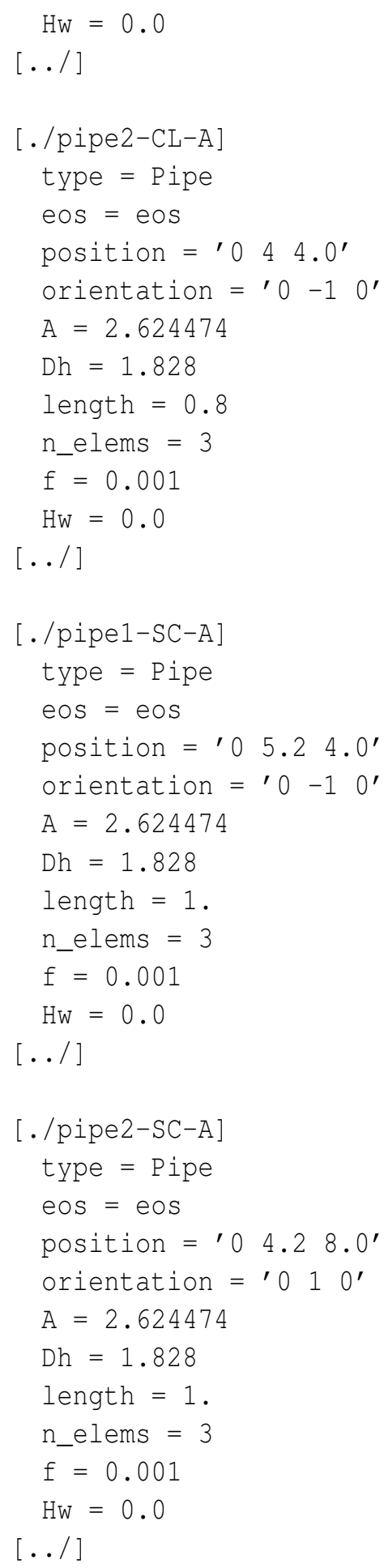




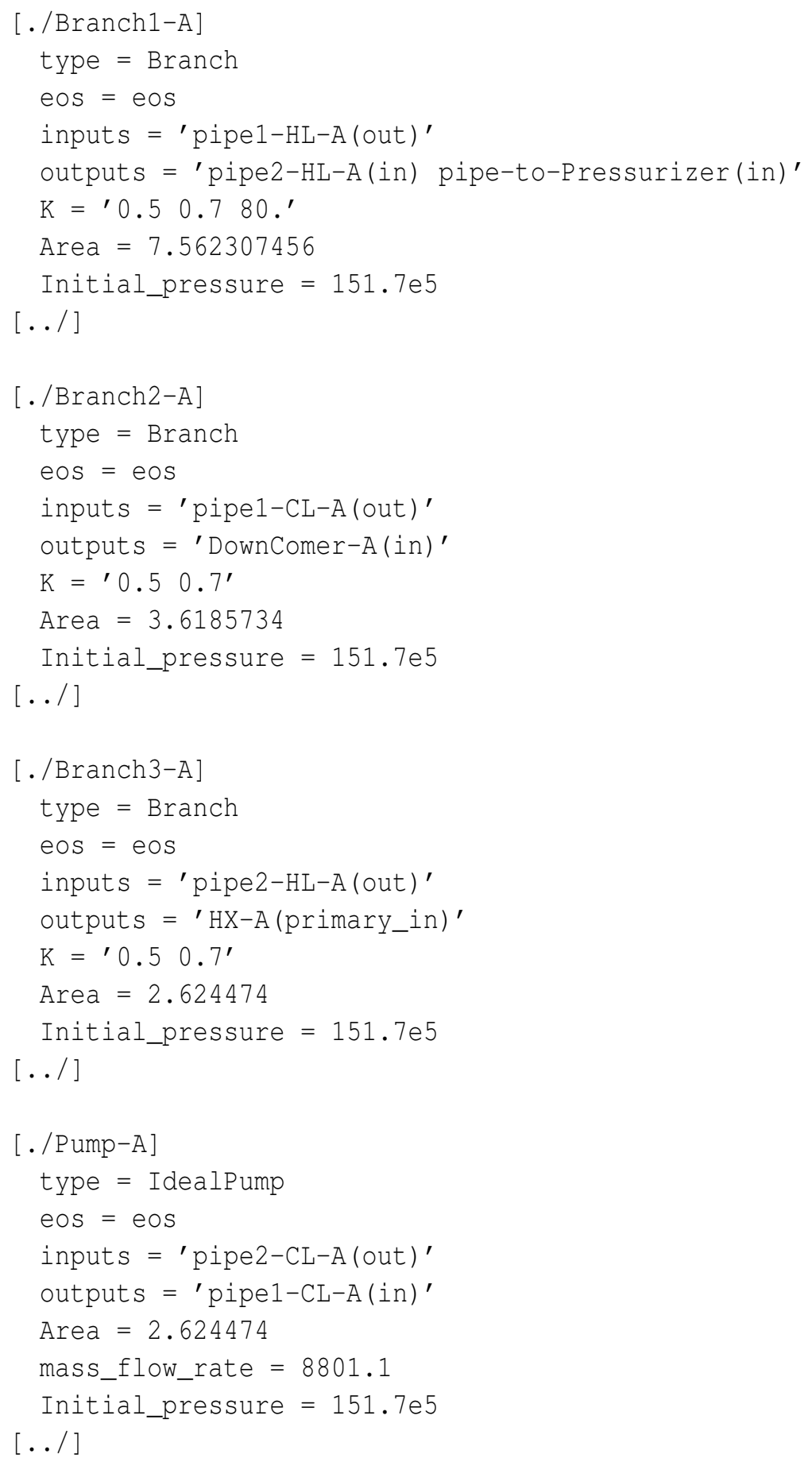




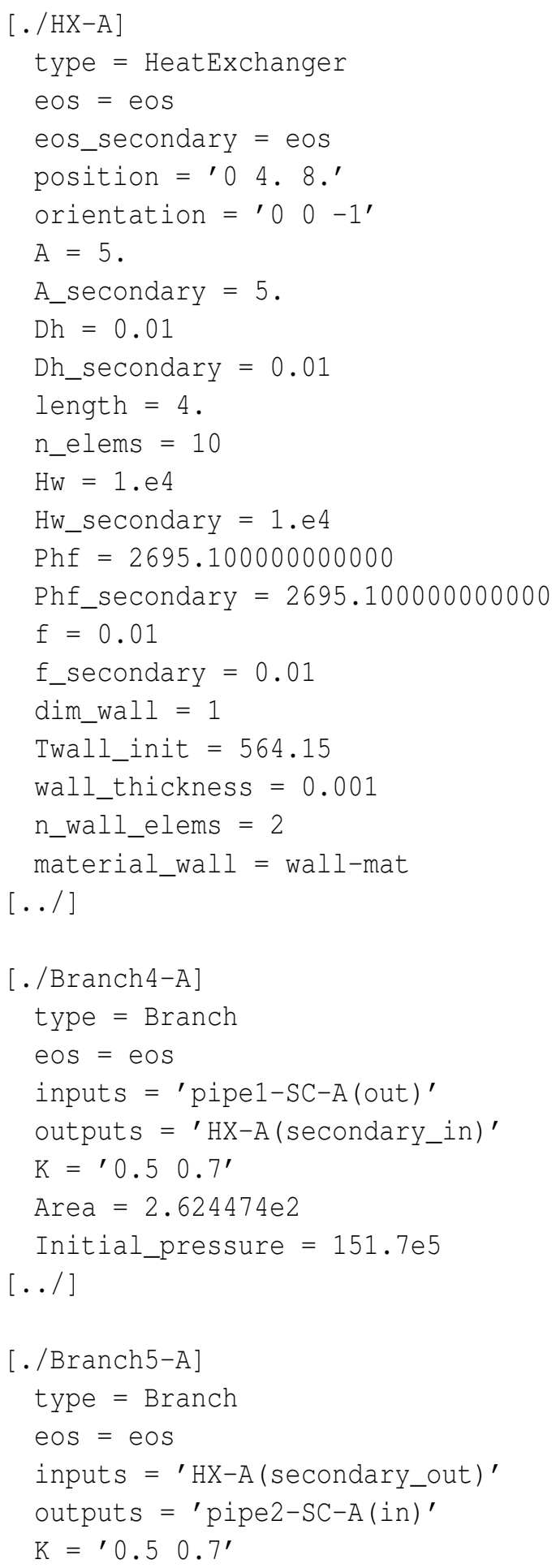




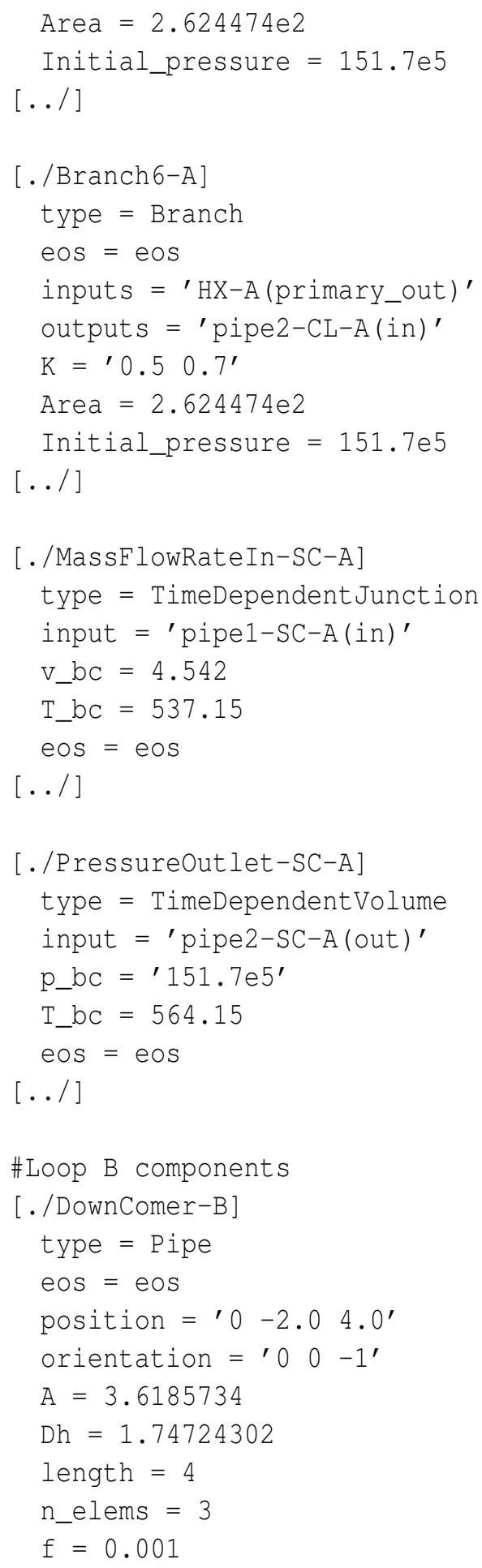




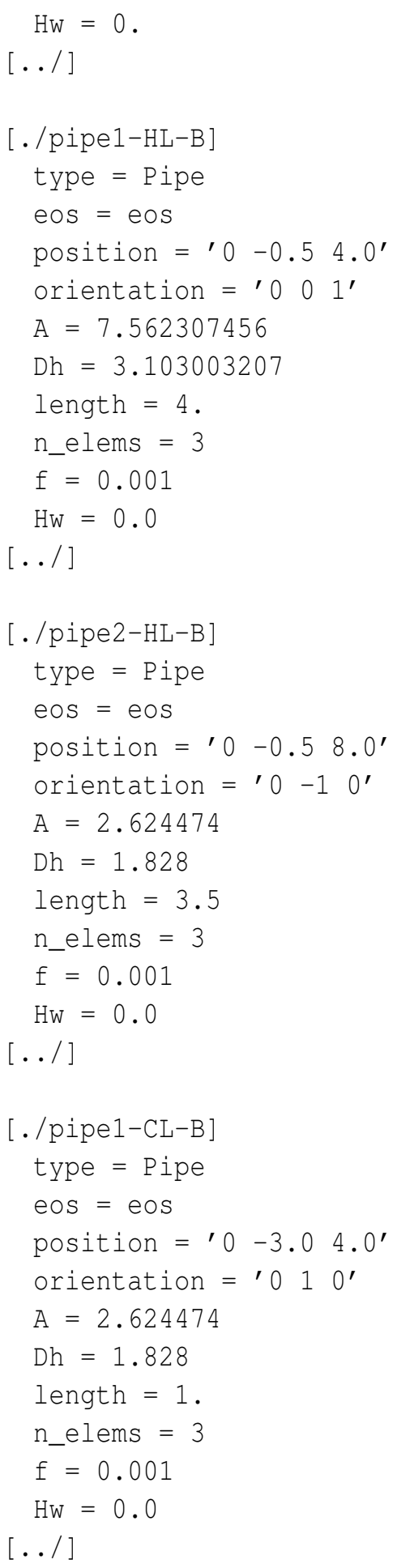




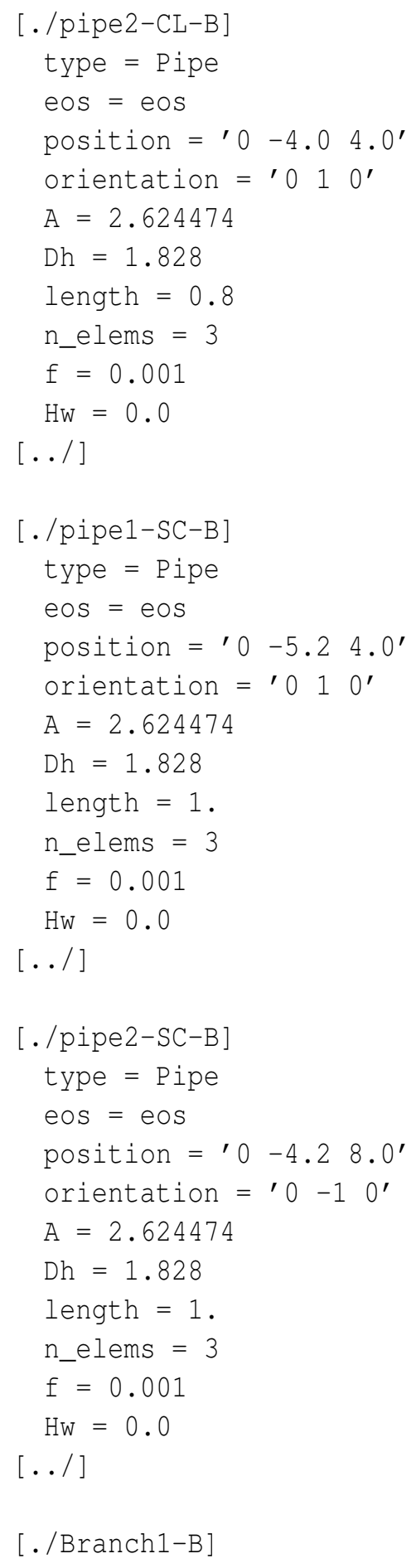




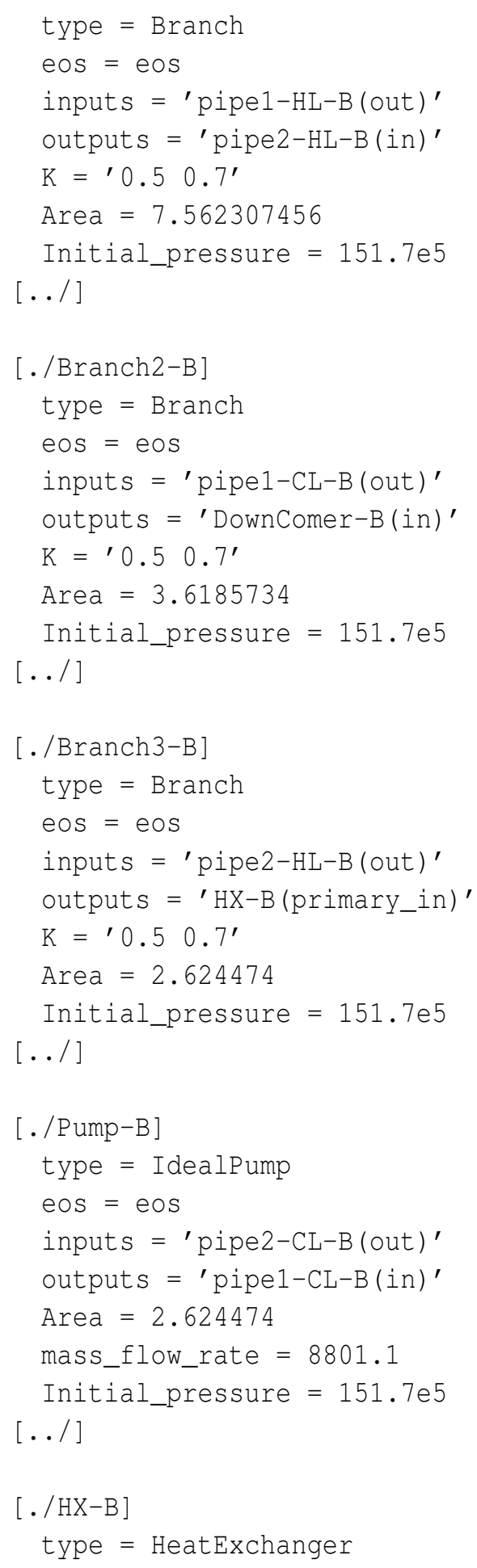




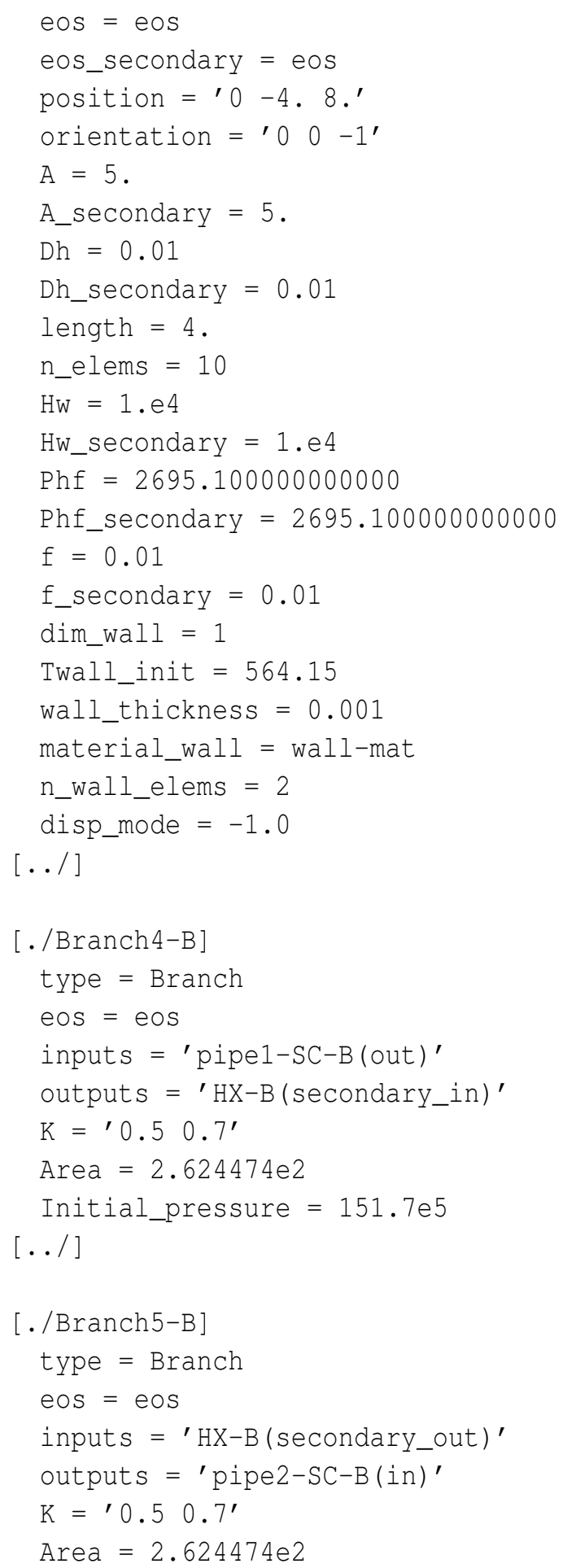




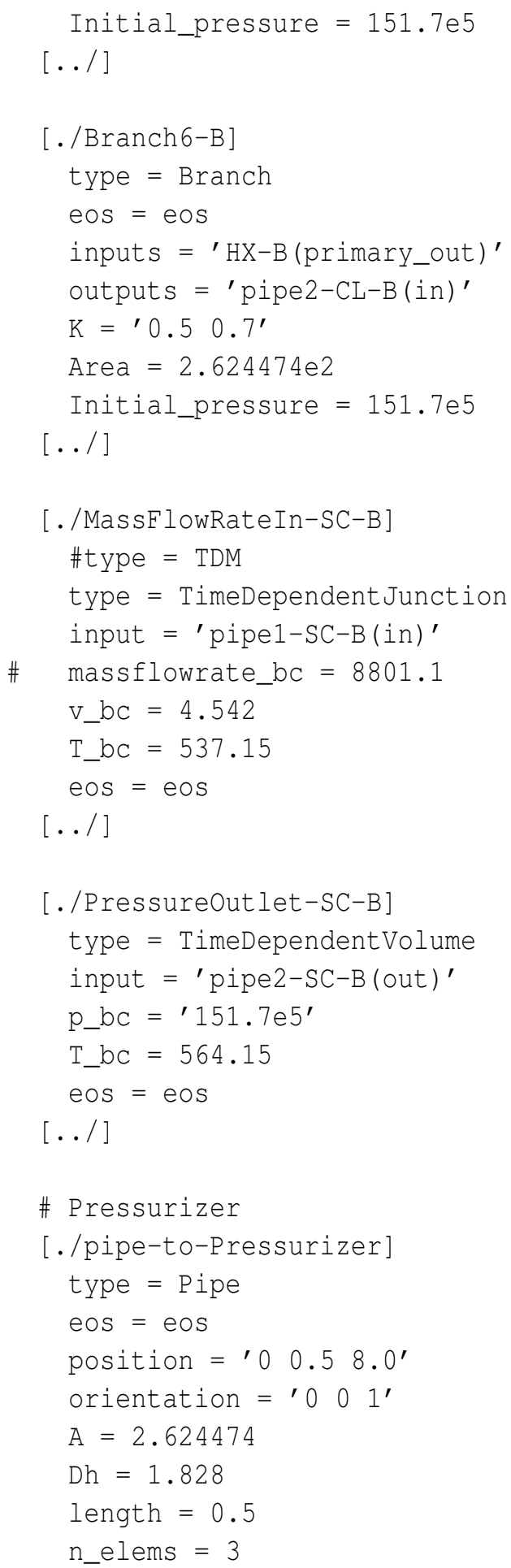




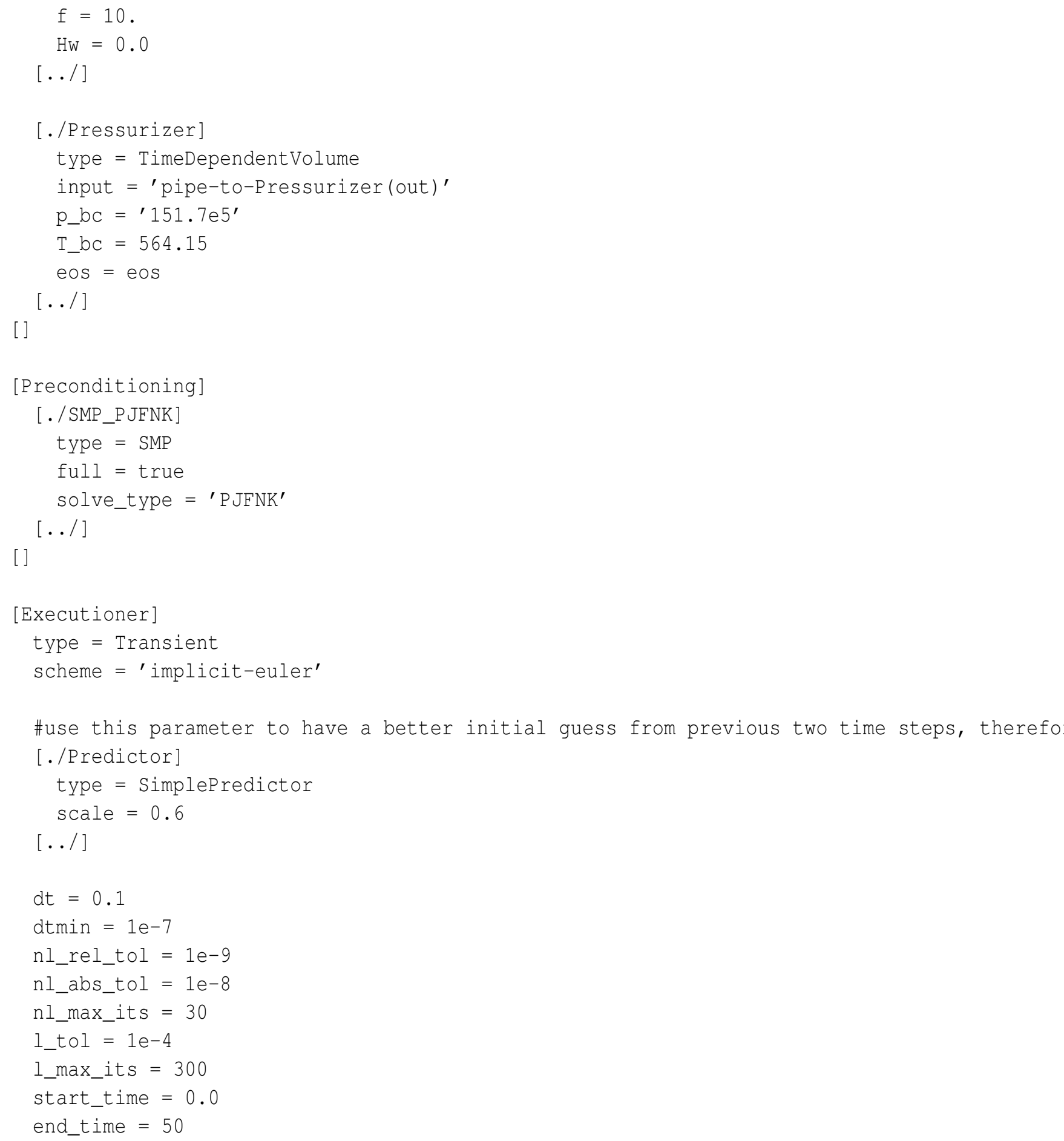




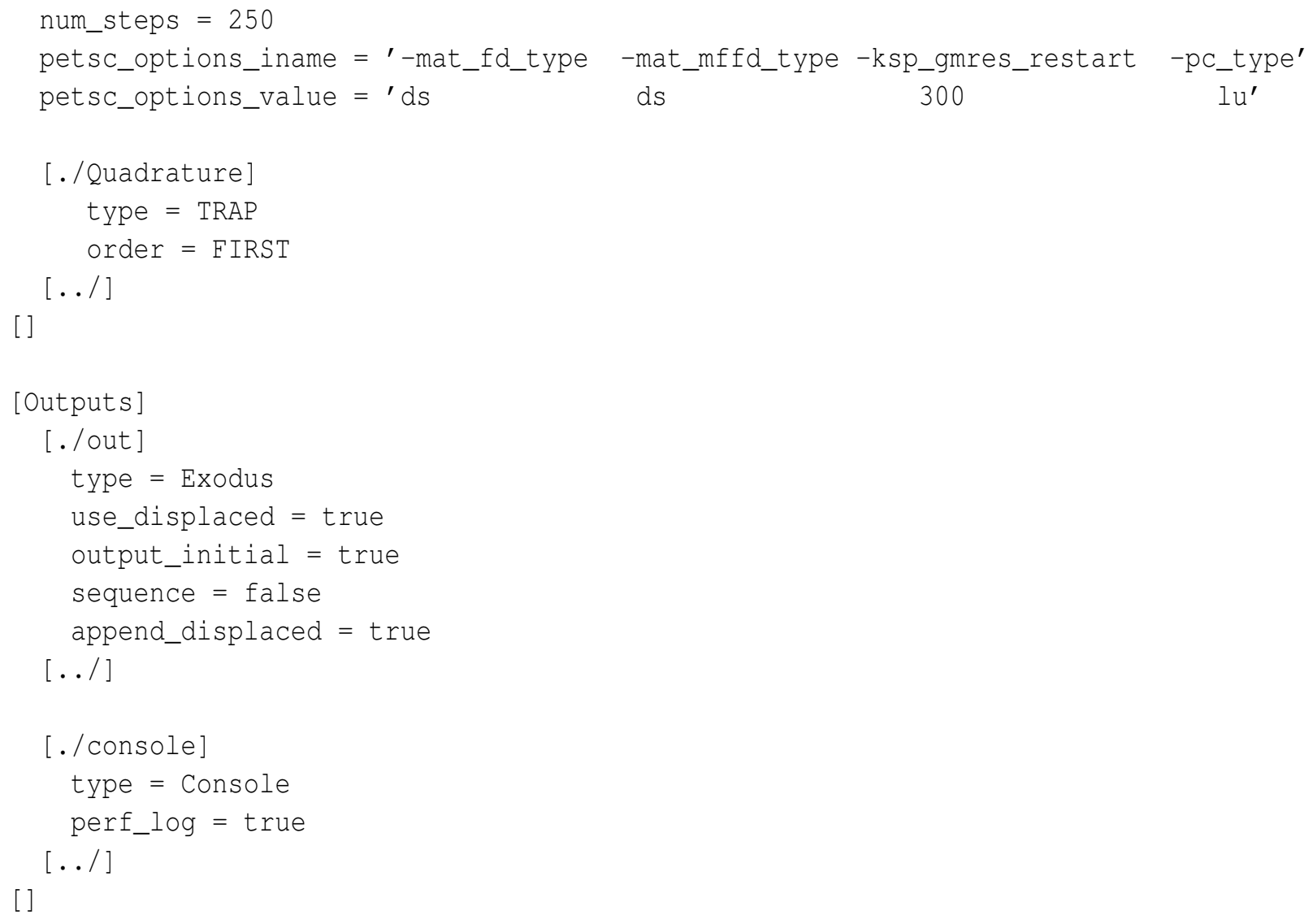

\subsubsection{Description of the Input File}

Most of the input parameters have been explained in the previous examples. Only a few need some explanations here.

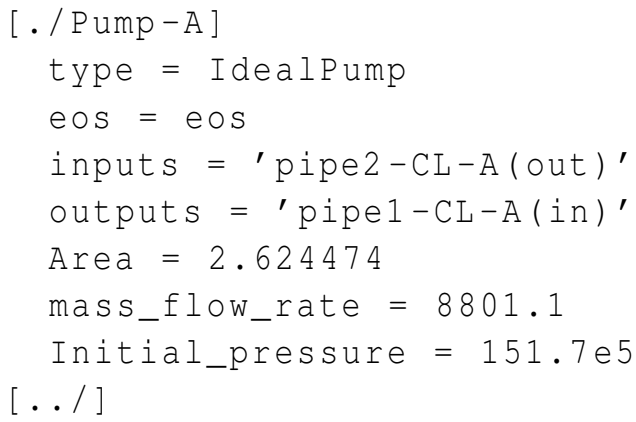


type

eos

inputs

outputs

Area

mass_flow_rate

The ideal pump component is used here. Please note that Ideal Pump component is an interim model. It merely provides a prescribed mass flow rate to the pipes with this component. This component will eventually be replaced with a realistic pump model.

Name of equation of state to be used by IdealPump.

The inlet of the ideal pump connects to the outlet of pipe2-CL-A.

The outlet of the ideal pump connects to the inlet of pipe1-CL-A.

The reference flow area of the ideal pump.

Initial_pressure The prescribed initial preesure of the ideal pump.

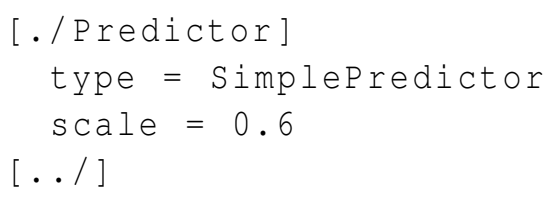

The prescribed mass flow rate generated by the ideal pump.

[./Predictor] Subblock for predictor. The predictor provides a better initil guess from the solution of previous two old time steps. It reduces the number of nonlinear iterations.

type The SimplePredictor uses an algorithm that will predict the next solution based on previous solutions. The algorithm is: $\operatorname{sol}^{n+1}=s o l^{n}+\frac{s o l^{n}-s o l^{n-1}}{\delta t^{n}} *$ $\delta t^{n+1} *$ scale.

scale The scale factor used in the SimplePredictor calculation. 


\subsection{Example 12: A Simplified Primary System Model of a Boiling Water Reactor}

\subsubsection{Problem Description}

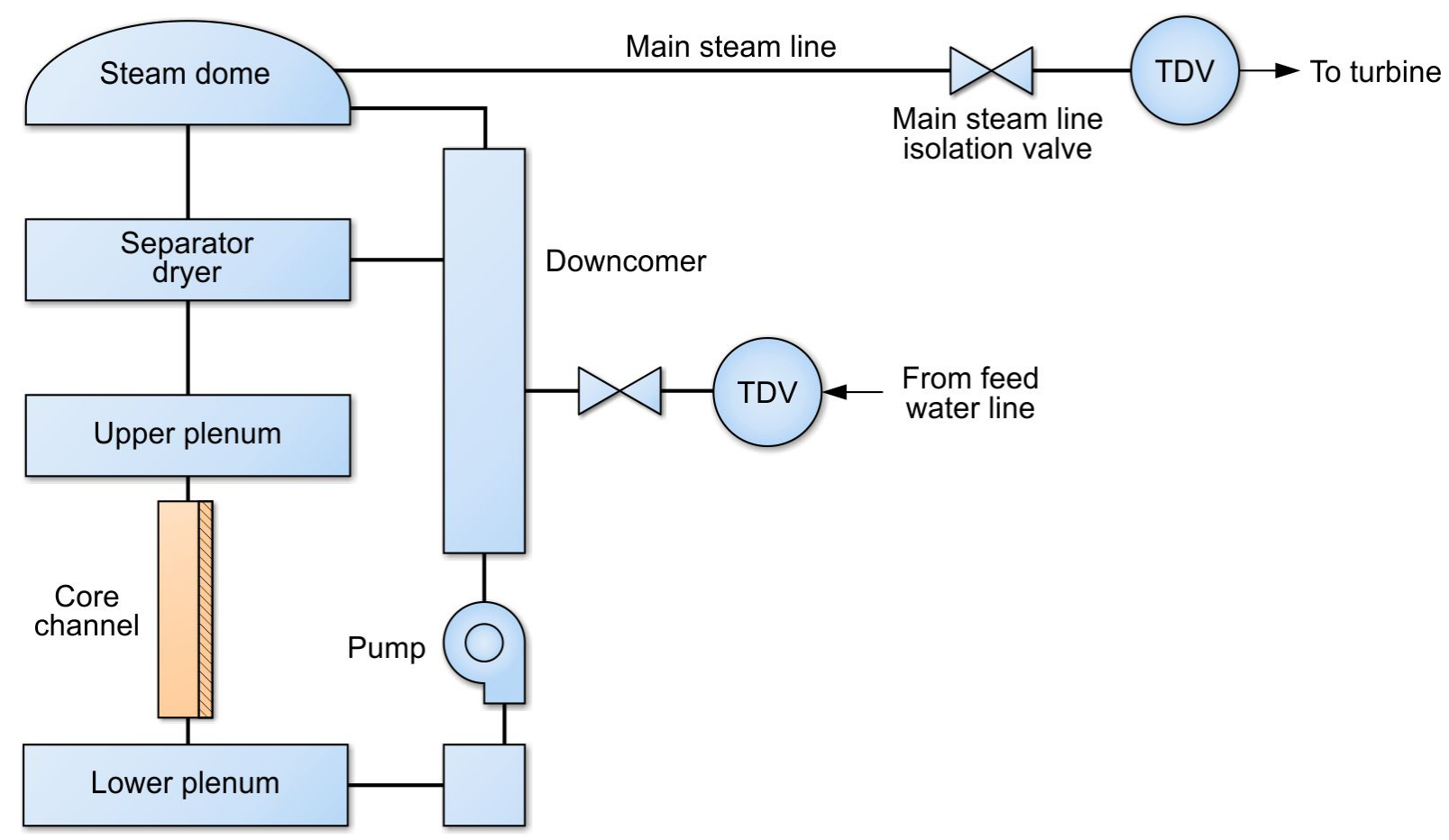

Figure 12. Diagram of a simplified primary system of a BWR model

This example is a simplified primary system of a boiling water reactor. The model consists of down comer, lower plenum, reactor core, upper plenum, separator dryer, steam dome, main steam line, feedwater line and the primary pump model. One core channel is used to represent the entire reactor core. The lower plenum, upper lenum and steam dome are modeled with volume branch models. External to the reactor vessel, the main steam line is connected to the steam dome. A time dependent volume is attached to the main steam line to provide the necessary boundary conditions for the steam flow. A feedwater line is connected to the downcomer model. A time dependent volume is attached to the feedwater line to provide the necessary boundary conditions for the feedwater. Notably missing from this simplified BWR model are the jet pumps and the recirculation loops that 
allow the operator to vary coolant flow rate through the core and change the reactor power. Instead, a pump model is used to represent the functions of the jet pumps and the recirculation loops.

\subsubsection{Input File}

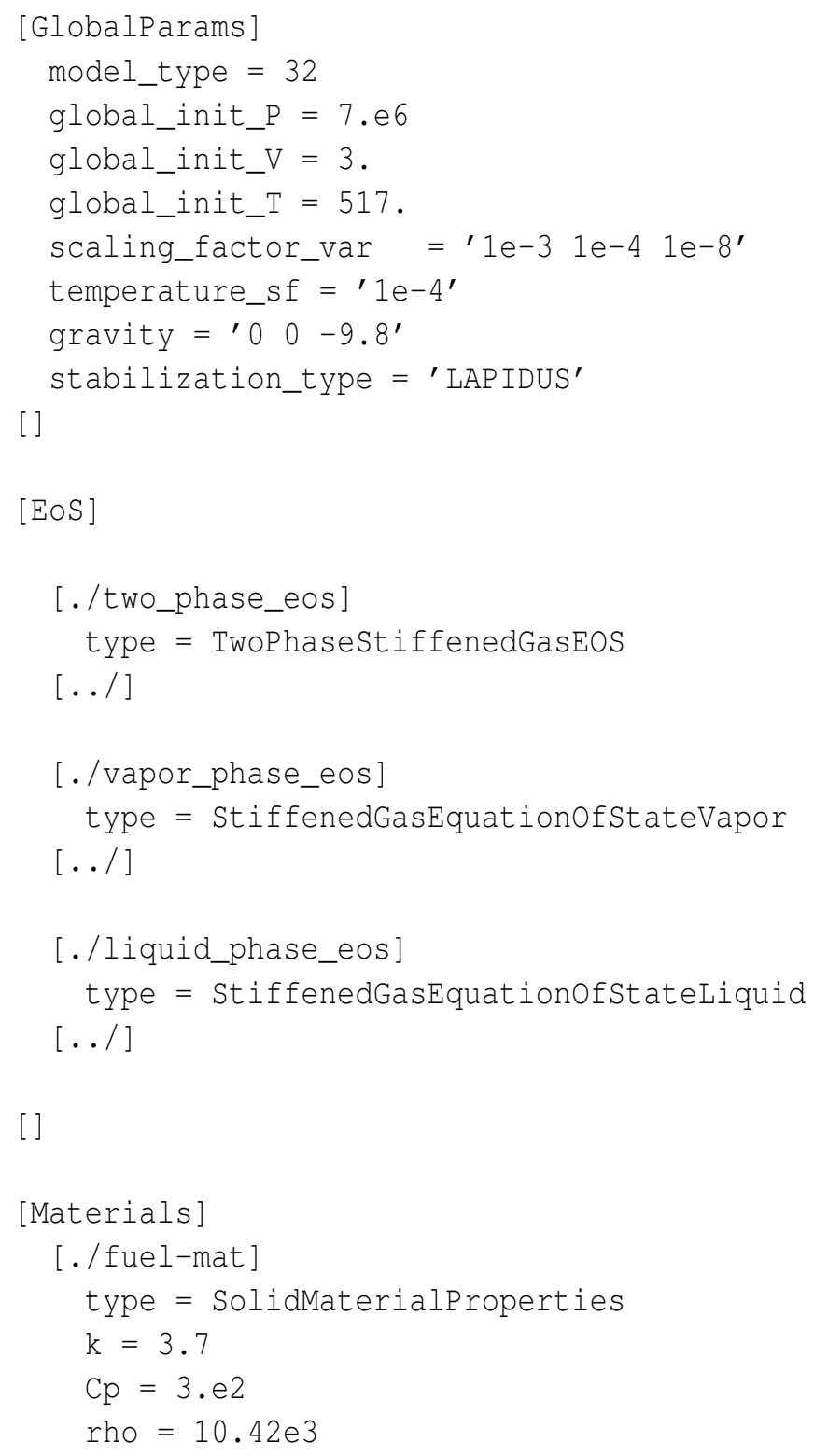




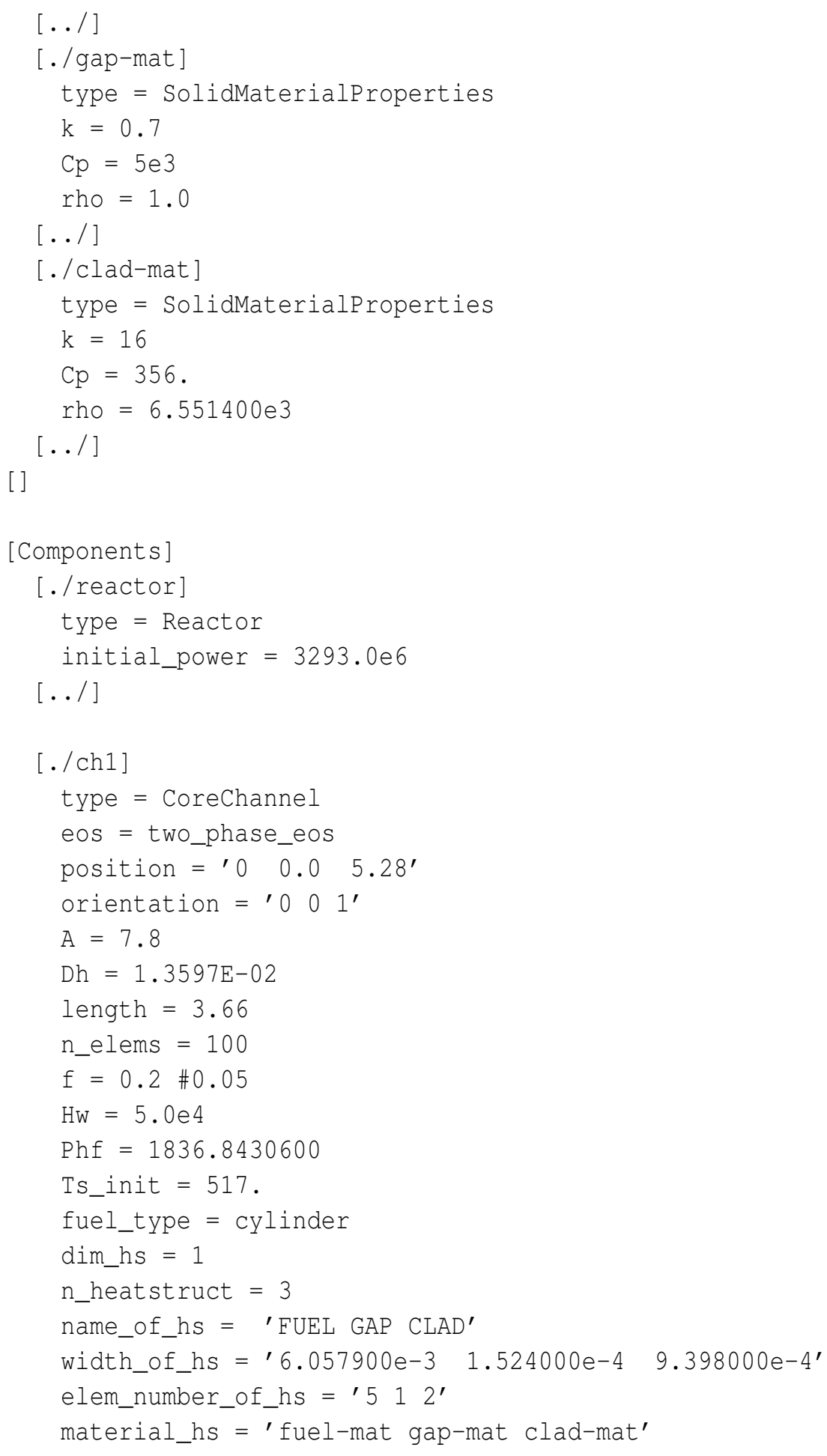




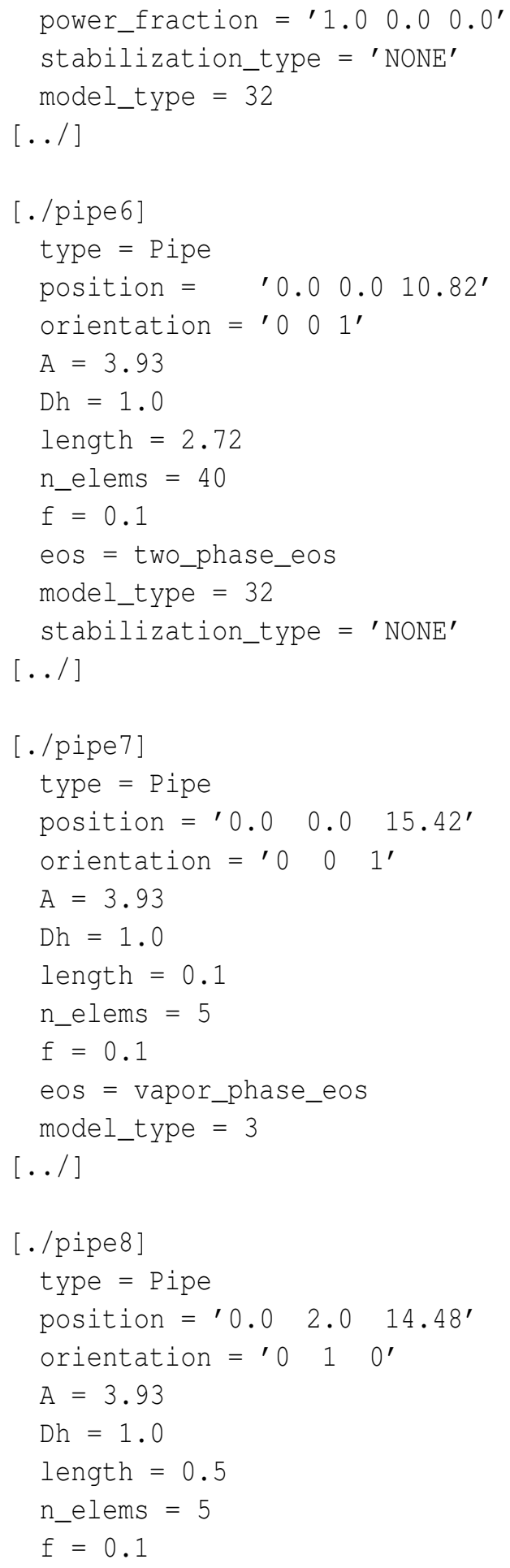




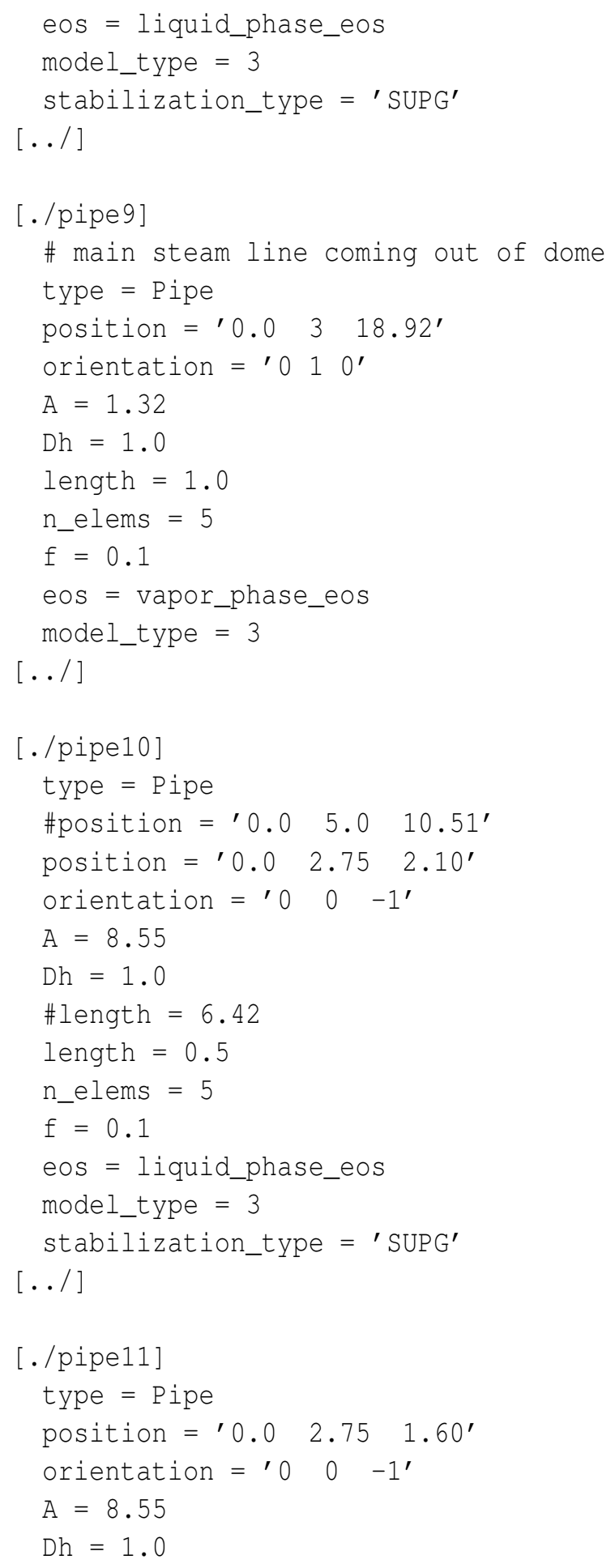




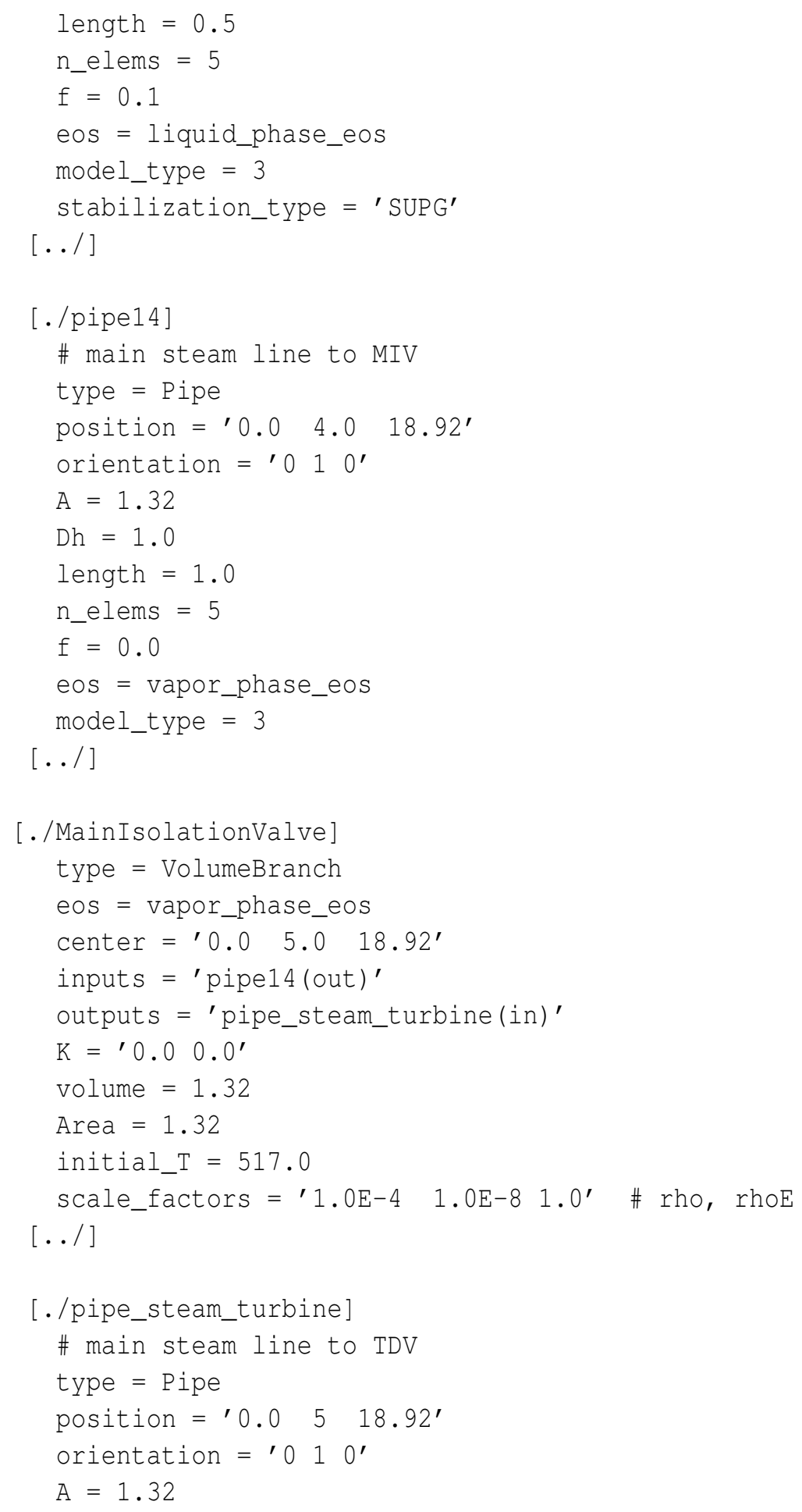




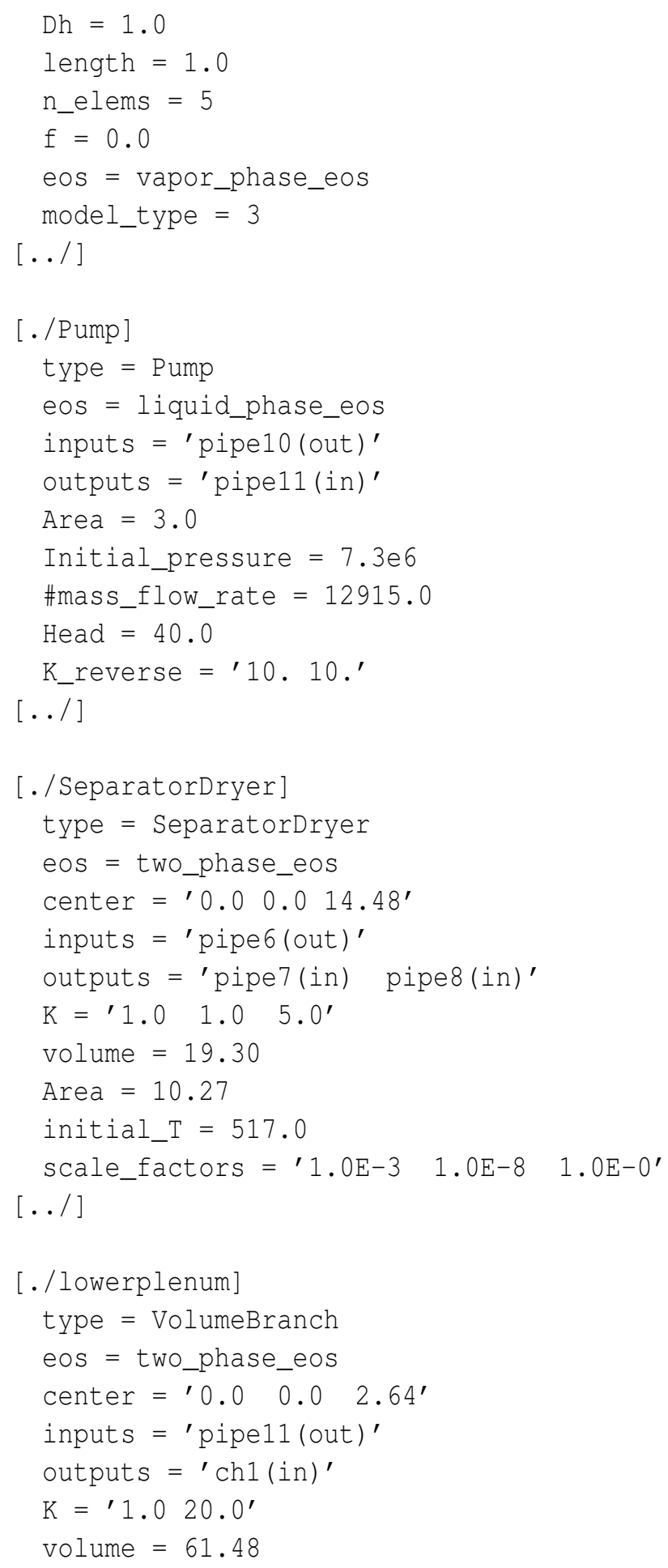




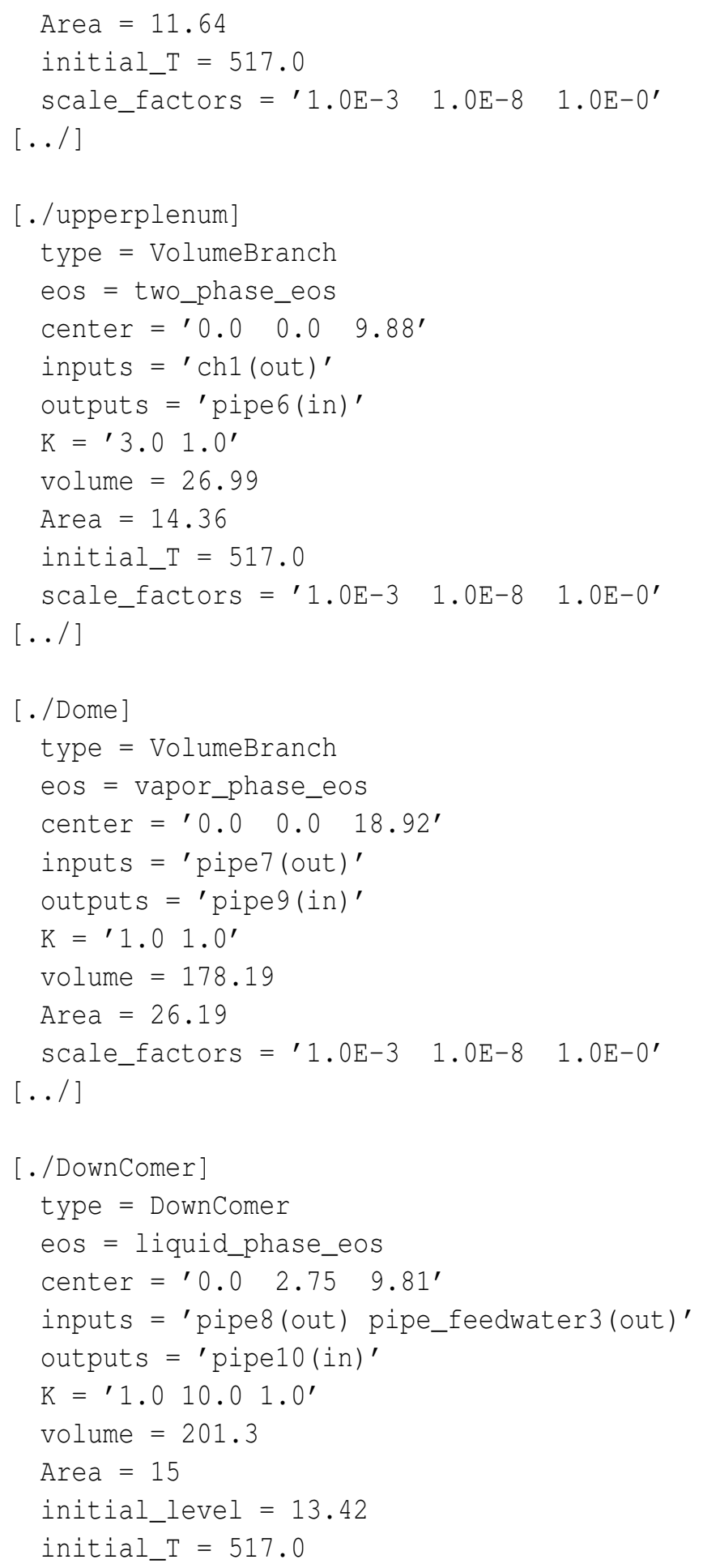




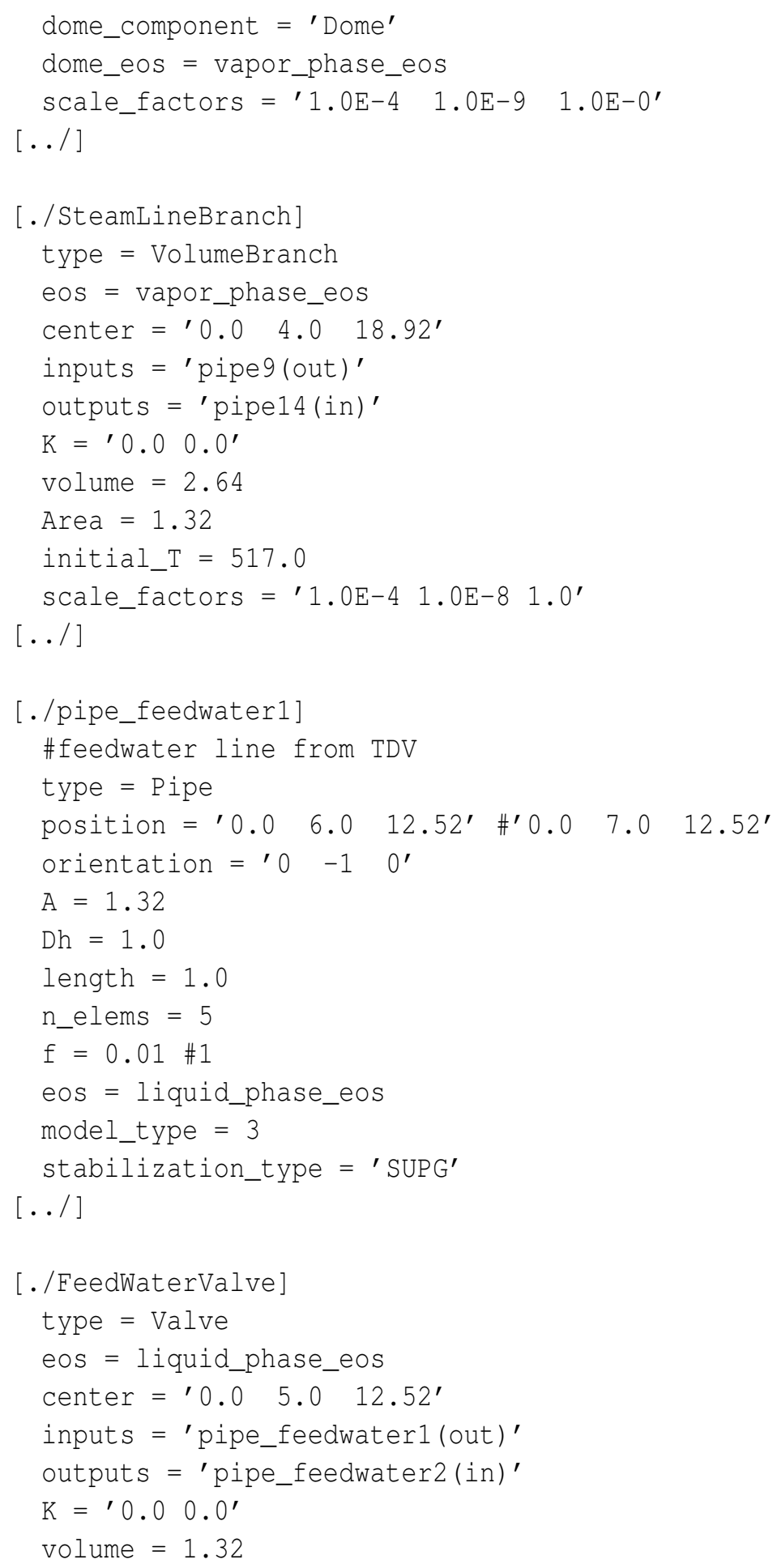




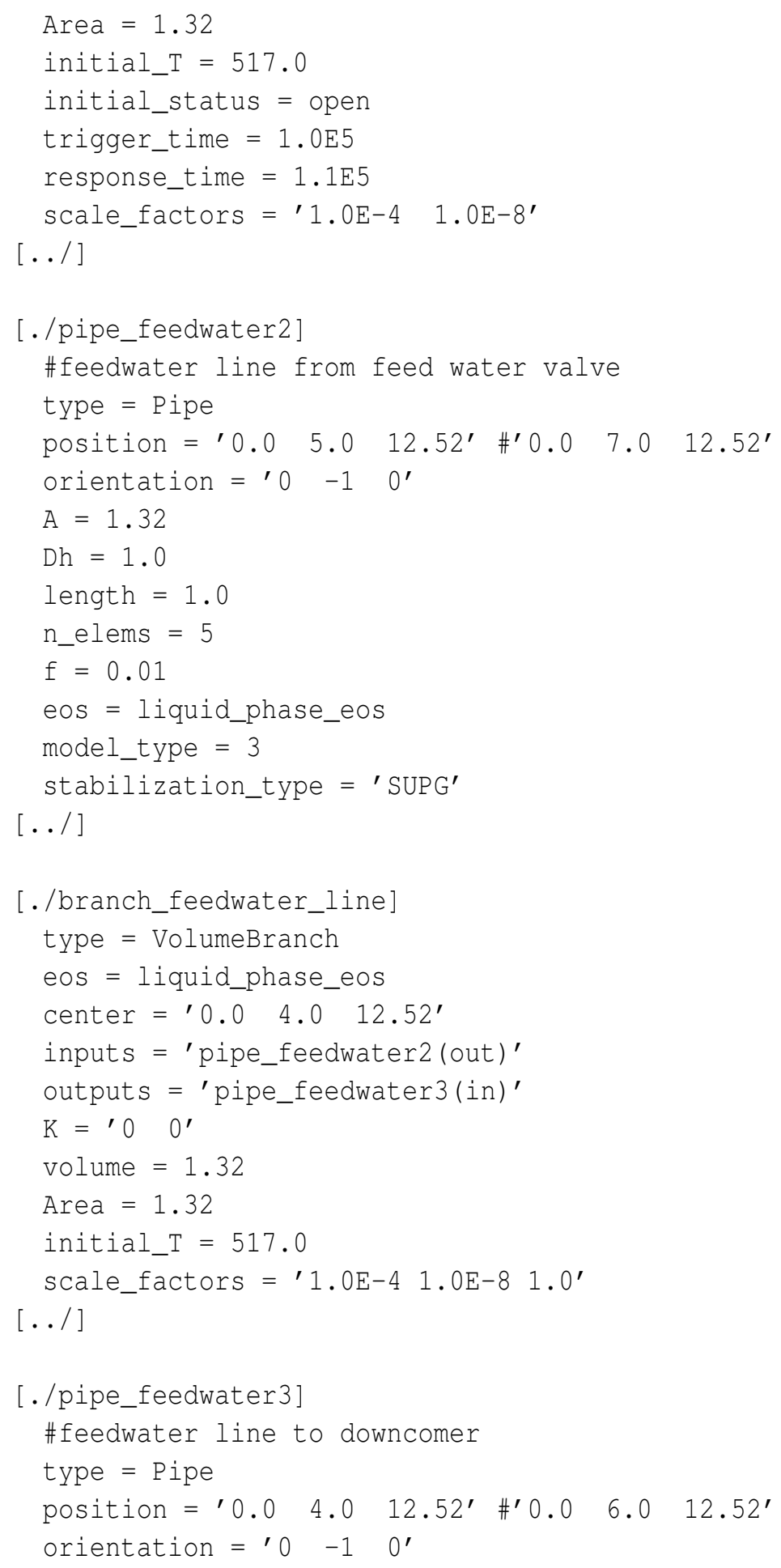




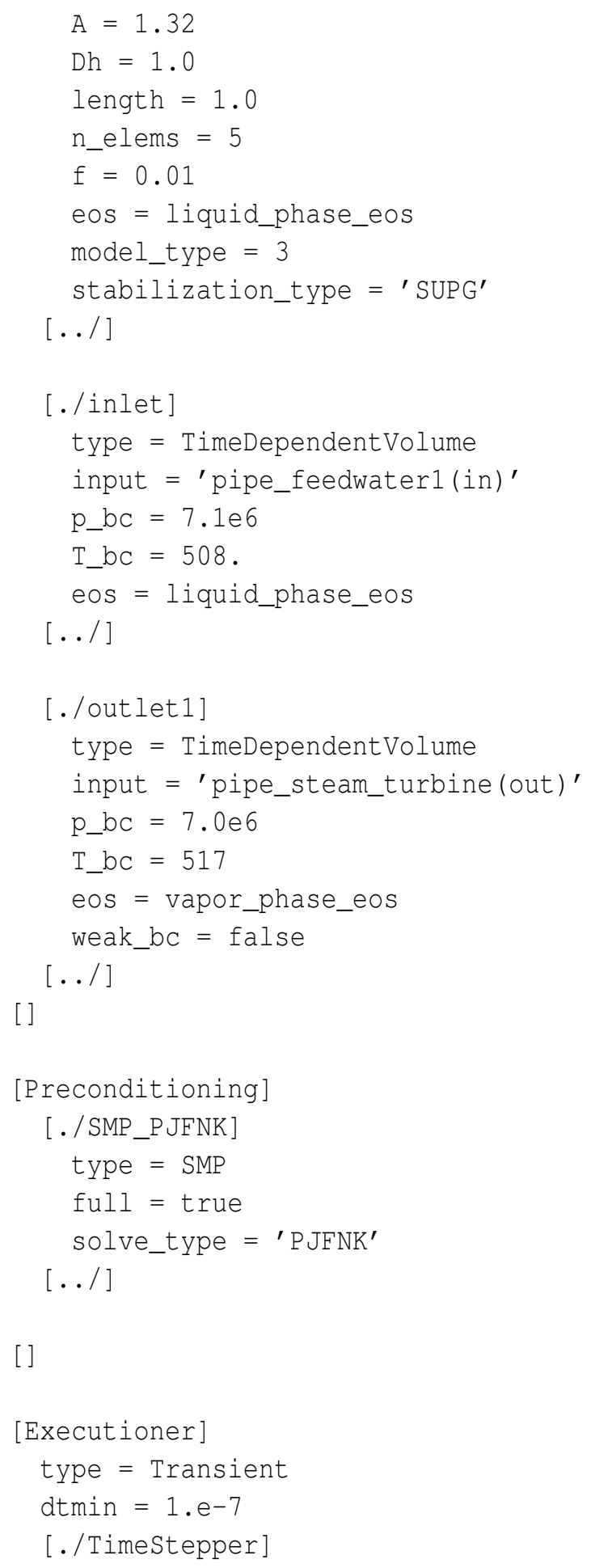




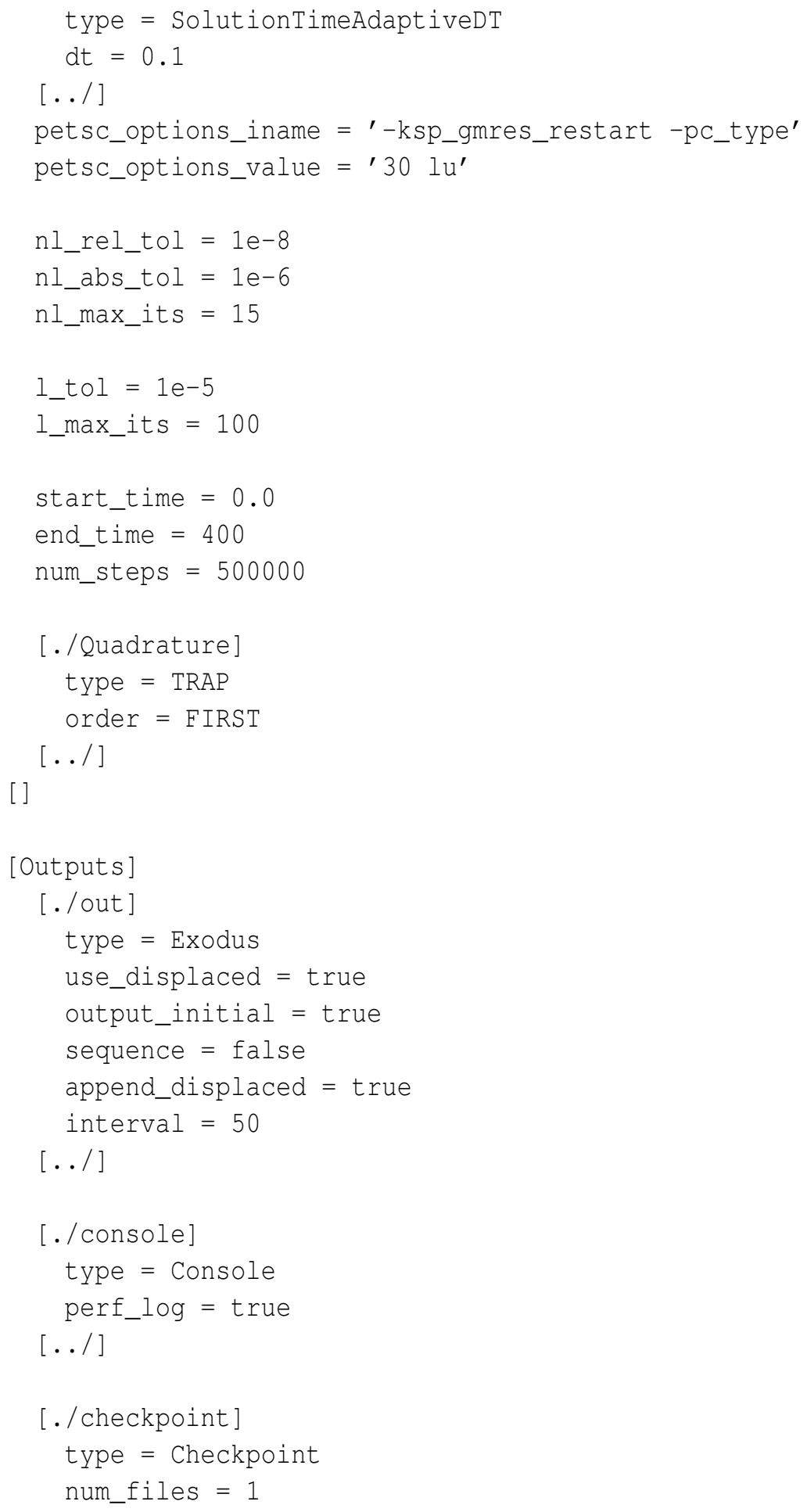




\subsubsection{Description of the Input File}

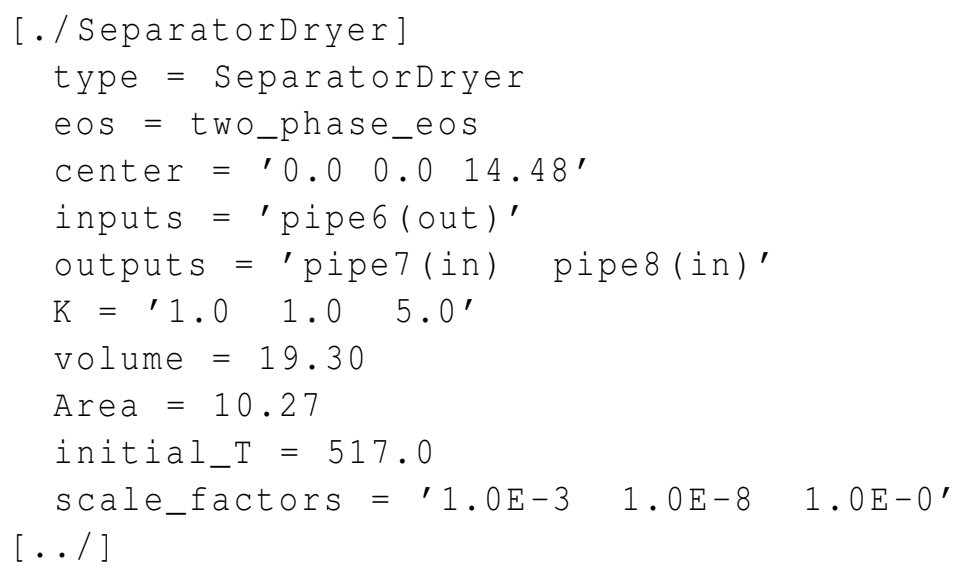



component.

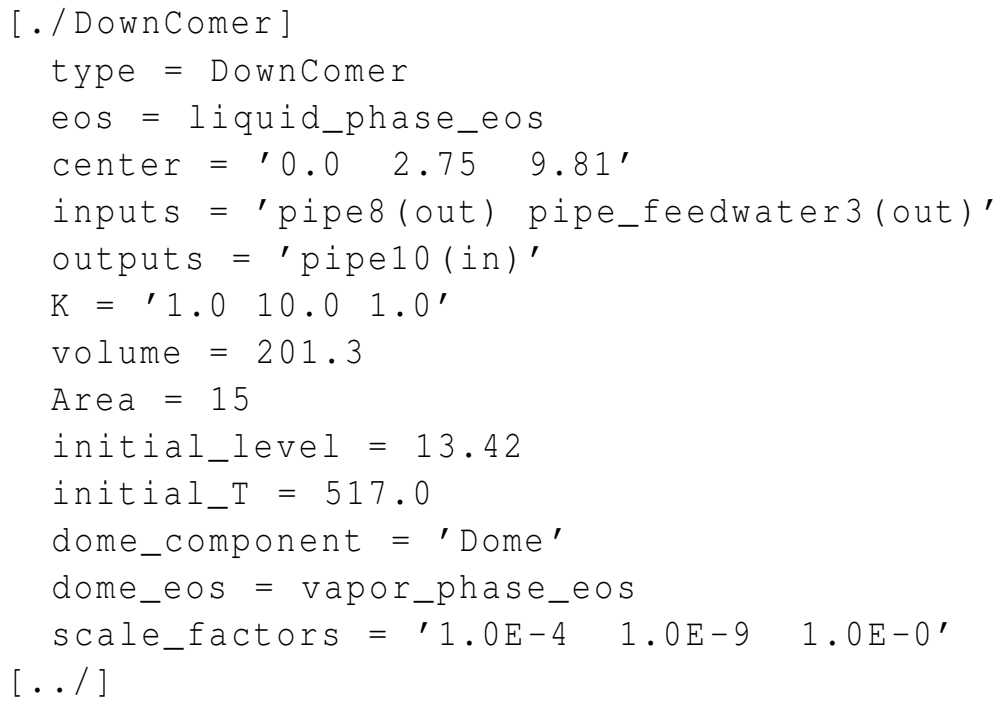


initial_T

dome_component

dome_eos

scale_factors
The initial temperature of the down comer component.

Name of the steam dome component to provide the dome pressure to down comer.

Name of the equation of state to be used by the steam dome component.

The saling factors for the primary variables of the down comer component.

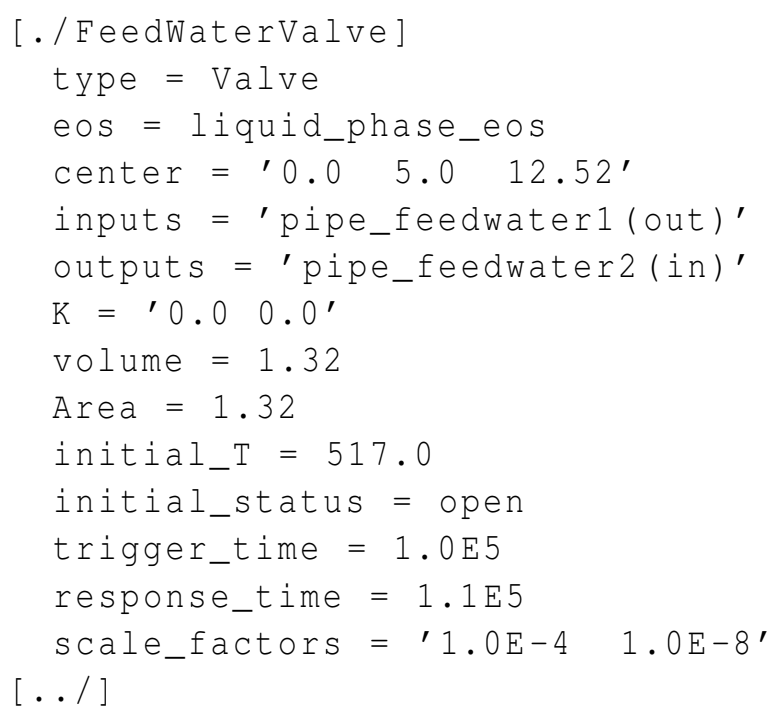

[./FeedWater] Subblock for the valve component.

type The component type is Valve.

eos Name od the equation of state to be used by the valve component.

center The $(x, y, z)$ coordinate of the center of the valve component.

inputs The inlet of valve connects with the outlet of pipe_feedwaterl.

outputs The outlet of valve connects with the inlet of pipe_feedwater2.

K The form loss coefficients at the junctions with the pipes. 
volume

Area

initial_T

initial_status

trigger_time

response_time

scale_factors
The volume of the valve component.

The reference flow area of the valve component.

The initial temperature of the valve component.

The initial status of the valve is at the open position.

The time at which the valve open/close action is triggered.

The time at which the valve open/close action is complete.

The saling factors for the primary variables of the valve component.

[./checkpoint ]

type $=$ Checkpoint

num_files $=1$

$[\ldots /]$

[./checkpoint] Subblock for check point. This is used to create restart files for subsequent runs.

num_files $\quad$ Number of restart files to save. 


\section{References}

[1] D. A. Knoll and D. E. Keyes, "Jacobian-free Newton-Krylov methods: a survey of approaches and applications," Journal of Computational Physics, vol. 193, pp. 357-397, Jan. 2004. http: //dx.doi.org/10.1016/j.jcp.2003.08.010.

[2] P. N. Brown and A. C. Hindmarsh, "Matrix-free methods for stiff systems of ODEs," SIAM J. Numer. Anal., vol. 23, pp. 610-638, June 1986. http://www. jstor.org/stable/2157527.

[3] B. S. Kirk, J. W. Peterson, R. H. Stogner, and G. F. Carey, "libmesh: A C++ Library for Parallel Adaptive Mesh Refinement/Coarsening Simulations," Engineering with Computers, vol. 22, no. 3-4, pp. 237-254, 2006. http://dx. doi.org/10.1007/s00366-006-0049-3.

[4] S. Balay, W. D. Gropp, L. Curfman-McInnes, and B. F. Smith, "Efficient management of parallelism in object oriented numerical software libraries," in Modern Software Tools in Scientific Computing (E. Arge, A. M. Bruaset, and H. P. Langtangen, eds.), pp. 163-202, Birkhäuser Press, 1997.

[5] M. Heroux et al., "An overview of Trilinos," Tech. Rep. SAND2003-2927, Sandia National Laboratories, 2003.

[6] H. Zhang, L. Zou, D. Andrs, H. Zhao, and R. C. Martineau, "Point Kinetics Calculations with Fully Coupled Thermal Fluids Reactivity Feedback," in International Conference on Mathematics, Computational Methods \& Reactor Physics (M\&C 2013), (Sun Valley, Idaho, USA), May 5-9, 2013.

[7] L. Zou, J. Peterson, H. Zhao, H. Zhang, D. Andrs, and R. C. Martineau, "Solving Multi-Mesh Flow and Conjugate Heat Transfer Problems with RELAP-7," in International Conference on Mathematics, Computational Methods \& Reactor Physics (M\&C 2013), (Sun Valley, Idaho, USA), May 5-9, 2013.

[8] R. D. Falgout and U. M. Yang, "HYPRE: A Library of High Performance Preconditioners," in International Conference on Computational Science, pp. 632-641, 2002. 


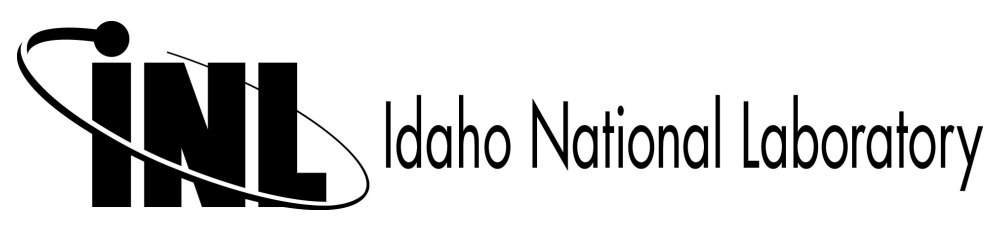

